\title{
Electromagnetic Fields Produced by Moving Sources in a Curved Beam Pipe
}

\author{
Shin-itiro Goto ${ }^{1}$ and Robin W Tucker ${ }^{1}$ \\ 1 Department of Physics, Lancaster University and the Cockcroft Institute, Daresbury
}

(Dated: October 31, 2018)

\begin{abstract}
A new geometrical perturbation scheme is developed in order to calculate the electromagnetic fields produced by charged sources in prescribed motion moving in a non-straight perfectly conducting beam pipe. The pipe is regarded as a perturbed infinitely long hollow right-circular cylinder. The perturbation maintains the pipe's circular cross-section while deforming its axis into a planar space-curve with, in general, non-constant curvature. Various charged source models are considered including a charged bunch and an off-axis point particle. In the ultra-relativistic limit this permits a calculation of the longitudinal wake potential in terms of powers of the product of the pipe radius and the arbitrarily varying curvature of the axial space-curve. Analytic expressions to leading order are presented for beam pipes with piecewise defined constant curvature modelling pipes with straight segments linked by circular arcs of finite length. The language of differential forms is used throughout and to illustrate the power of this formalism a pedagogical introduction is developed by deriving the theory ab-initio from Maxwell's equations expressed intrinsically as a differential system on (Minkowski) spacetime.

PACS numbers: 87.56.bd, 02.40.-k, 31.15.xp, 41.20.-q, 29.27.-a, 41.60.-m, 41.75.-i
\end{abstract}

\section{INTRODUCTION}

Considerable activity is being devoted to the design of advanced machines that can produce pulsed sources of intense focussed electromagnetic radiation. Such sources offer unprecedented opportunities for probing the temporal and spatial microstructure of processes in Nature. Many designs rely on being able to control the motion of ultrarelativistic electron beams by external fields in beam pipes with non-uniform spatial curvature. The production of femtosecond radiation pulses requires high peak electric currents and the maintenance of low emittance electron beams for the self-amplified spontaneous emission of X-rays demands extreme design criteria in order to sustain beam stability in the presence of radiation back-reaction on accelerated sources.

A direct analytic approach to this electrodynamic problem via the coupled system of Maxwell's field equations and the equations of motion for the particle beams encounters difficult problems due to non-linearities and retardation effects [1, 2, 3]. A direct approach using statistical methods suffers from similar complications. Numerical approximations based on these equations exist but few are able to address the full complexities encountered in a realistic situation. In particular even when non-linearities are deemed negligible, the effects of (conducting) boundaries on the accelerating source via the radiation fields are often ignored in an attempt to build tractable models and the effects of pipe curvature are often restricted to those produced by motion in arcs of circles [4]. Although insight can be gained from such modelling we feel that much remains to be understood in more general scenarios.

In this paper we explore a new geometrical perturbation scheme that addresses some aspects of the general electrodynamical problem of charged sources in prescribed arbitrary motion moving in a non-straight beam pipe. In the analysis below the pipe will be regarded as a perturbed infinitely long hollow right-circular cylinder. The perturbation will maintain the pipe's circular cross-section while deforming its axis into a planar space-curve with, in general, non-constant curvature. It is assumed that the curvature $\kappa(z)$ depends on the arc-length $|z|$ of the planar space-curve and tends to zero as $z \rightarrow \pm \infty$. Furthermore if the cylinder has fixed radius $a$ we require that $|\kappa(z) a| \ll 1$ for the perturbation analysis to be effective. Under these conditions the initial-boundary value problem for the Maxwell fields in a perfectly conducting hollow perturbed cylinder given prescribed currents will be considered. The source currents will excite superpositions of electromagnetic modes of the empty perturbed cylinder as well as generating their own "self-fields". The latter will include acceleration-fields induced by the motion of the sources in the curved regions of the beam pipe. A perturbation scheme will be established to calculate all fields in the pipe as a perturbation expansion in powers of $a \kappa$. Mode expansions based on Dirichelet and Neumann eigen-functions of the Laplacian for a circular disc domain can be used to reduce the general problem at each order to a two-dimensional linear telegraph-type equation with prescribed sources. The general causal solution of this equation is known and from it the complete set of electromagnetic fields can be constructed. Thus the perturbed beam pipe impedances can be found. Furthermore the fields arise naturally in a space-time description and thereby offer direct input into "leap-frog" coding schemes that couple the Maxwell sector to the equations of motion of the sources.

In section 2 the notation used in the paper is established and illustrated by means of a pedagogic review of exterior methods used for solving Maxwell boundary value problems in sections 3 and 4. Section 5 deals with the introduction of Dirichelet and Neumann modes used to reduce the Maxwell system with perfectly conducting boundaries to a telegraph 
type equation in two dimensions. Section 6 uses a geometric perturbation approach to explore the dependence of the radiated power from a smooth longitudinal convective current on local beam-pipe curvature. Section 7 deals with a moving point charge source and discusses in some detail radiant instantaneous power. This permits a perturbative calculation of the ultra-relativistic wake potentials in a planar pipe with arbitrary curvature and explicit analytic expressions can be found in the case where the curvature is piecewise constant modelling pipes with straight segments linked by circular arcs of finite length. Finally section 8 considers the radiation from smooth convected localised bunches with fixed total charge while the Appendices tabulate coupling coefficients and transfer kernels used in the main text.

\section{NOTATION}

The exterior calculus of differential forms offers a versatile and powerful means for analysing Maxwell's equations [5, 6]. The notation used below follows standard conventions for a manifold $M$ with a metric tensor field. Thus $\Gamma T M$ denotes the set of vector fields and $\Gamma \Lambda^{p} M$ the set of $p$-form fields on $M$. Metric duals with respect to any metric tensor $g$ are written with a tilde so that $\widetilde{X}=g(X,-) \in \Gamma \Lambda^{1} M$ for $X \in \Gamma T M$ and $\widetilde{\alpha}=g^{-1}(\alpha,-) \in \Gamma T M$ for $\alpha \in \Gamma \Lambda^{1} M$. The Hodge dual map associated with $g$ is denoted by a star so that the canonical $n$-form measure ("volume element") on an $n$-dimensional manifold $M$ is the image of 1 under the Hodge map. In 4 -dimensional spacetime we adopt the flat Minkowski metric. In a 3-dimensional space we adopt the Euclidean flat metric and regard time as an evolution parameter for forms in three dimensions. In a 2-dimensional space we adopt the Euclidean flat metric and regard time and a longitudinal coordinate as parameters for forms in two dimensions. One must then distinguish notationally between the different metrics introduced and their associated Hodge maps. However for any manifold $M$ with Hodge map $\star$ one always has the standard relations

$$
\begin{gathered}
\Phi \wedge \star \Psi=\Psi \wedge \star \Phi, \quad \text { for } \quad \Phi, \Psi \in \Gamma \Lambda^{p} M \\
i_{X} \star \Phi=\star(\Phi \wedge \tilde{X}), \quad \text { for } \quad X \in \Gamma T M, \Phi \in \Gamma \Lambda^{p} M
\end{gathered}
$$

where $i_{X}$ denotes the interior (contraction) operator on forms.

Maxwell's equations find their most cogent formulation as a theory of 2-forms on spacetime modelled on a space and time oriented 4-dimensional manifold with a metric tensor field $g$ of Lorentzian signature $(-,+,+,+)$. On such a spacetime $M$ the set $\left\{e^{0}, e^{1}, e^{2}, e^{3}\right\}$ will denote a local $g$-orthonormal coframe (a linearly independent collection of 1-forms). The Hodge map associated with the Lorentzian metric $g$ will be denoted by $\star$. Then

$$
\begin{aligned}
& \star i_{X} \Phi=-\star \Phi \wedge \tilde{X}, \quad \text { for } \quad X \in \Gamma T M, \Phi \in \Gamma \Lambda^{p} M \\
& \star \star \Phi=(-1)^{p+1} \Phi, \quad \text { for } \Phi \in \Gamma \Lambda^{p} M
\end{aligned}
$$

For manifolds with a Euclidean signature and different dimensions these last two relations change as will be indicated for three and two dimensional spaces below. Finally note that for all $n$-dimensional manifolds of any signature one has the useful results:

$$
\begin{gathered}
i_{X} \Phi \wedge \Psi=(-1)^{p+1} \Phi \wedge i_{X} \Psi, \quad \text { for } \quad \Phi \in \Gamma \Lambda^{p} M, \\
\Psi \in \Gamma \Lambda^{q} M, p+q \geq n+1 \\
d \Phi \wedge \Psi=(-1)^{p+1} \Phi \wedge d \Psi+d(\Phi \wedge \Psi), \quad \text { for } \Phi \in \Gamma \Lambda^{p} M, \\
\Psi \in \Gamma \Lambda^{q} M
\end{gathered}
$$

As a notational convenience the expression $\Psi$ below implies that $\Psi \in \Gamma \Lambda^{q} N$ is a differential $q$-form on $N$ where the manifold $N$ follows from the context.

\section{ELECTROMAGNETIC FIELDS IN SPACETIME}

Maxwell's equations for an electromagnetic field in an arbitrary medium can be written

$$
d F=0 \quad \text { and } \quad d \star G=j,
$$


where $F \in \Gamma \Lambda^{2} M$ is the Maxwell 2-form, $G \in \Gamma \Lambda^{2} M$ is the excitation 2-form and $j \in \Gamma \Lambda^{3} M$ is the 3 -form electric current source 14]. To close this system, "electromagnetic constitutive relations" relating $G$ and $j$ to $F$ are necessary.

The electric 4-current $j$ describes both (mobile) electric charge and effective (Ohmic) currents in a conducting medium. The electric field $\mathbf{e} \in \Gamma \Lambda^{1} M$ and magnetic induction field $\mathbf{b} \in \Gamma \Lambda^{1} M$ associated with $F$ are defined with respect to an arbitrary unit future-pointing timelike 4 -velocity vector field $U \in \Gamma T M$ by

$$
\mathbf{e}=i_{U} F \quad \text { and } \quad c_{0} \mathbf{b}=i_{U} \star F .
$$

Thus $i_{U} \mathbf{e}=0$ and $i_{U} \mathbf{b}=0$.

Since $g(U, U)=-1$

$$
F=\mathbf{e} \wedge \widetilde{U}-\star\left(c_{0} \mathbf{b} \wedge \widetilde{U}\right)
$$

The field $U$ may be used to describe an observer frame on spacetime and its integral curves model idealised observers.

Likewise the displacement field $\mathbf{d} \in \Gamma \Lambda^{1} M$ and the magnetic field $\mathbf{h} \in \Gamma \Lambda^{1} M$ associated with $G$ are defined with respect to $U$ by

$$
\mathbf{d}=i_{U} G, \quad \text { and } \quad \mathbf{h} / c_{0}=i_{U} \star G .
$$

Thus

$$
G=\mathbf{d} \wedge \widetilde{U}-\star\left(\left(\mathbf{h} / c_{0}\right) \wedge \widetilde{U}\right)
$$

and $i_{U} \mathbf{d}=0$ and $i_{U} \mathbf{h}=0$. It may be assumed that a material medium has associated with it a future-pointing timelike unit vector field $V$ which may be identified with the bulk 4-velocity field of the medium in spacetime. Integral curves of $V$ define the averaged world-lines of identifiable constituents of the medium. A comoving observer frame with 4-velocity $U$ will have [15] $U=V$.

\section{TIME DEPENDENT MAXWELL SYSTEMS IN SPACE}

On any $n$-dimensional manifold a chart sets up a correspondence between points on some region (patch) on the manifold and a set on $\mathbb{R}^{n}$. Thus in a 2-dimensional patch let $\underline{\hat{\xi}}=\left(\xi^{1}, \xi^{2}\right)$ be a generic set of coordinates. Similarly let $\underline{\xi}=\left(\xi^{1}, \xi^{2}, \xi^{3}\right)$ denote coordinates on a patch of a 3 -dimensional manifold and $\xi=\left(\underline{\xi}\right.$, $\left.\xi^{0}\right)$ denote coordinates on a patch of 4 -dimensional spacetime.

Let $\boldsymbol{d}$ denote exterior differentiation in any domain of a Euclidean space with coordinates $\underline{\xi}$. Similarly let $\hat{\boldsymbol{d}}$ denote exterior differentiation in a patch with coordinates $\hat{\xi}$. A "moving" orthonormal (Cartan) coframe in flat spacetime with Minkowski metric $g$ is a set of (independent [16]) 1 -forms $\left\{e^{0}, e^{1}, e^{2}, e^{3}\right\}$ with $e^{0}$ timelike. In general this will depend on the choice of coordinates $\xi$ in the sense that its exterior derivative will not be zero. In the following we adopt an inertial frame with laboratory time $\xi^{0}=t$ and $e^{0}=c_{0} d t$ with $\left\{e^{1}, e^{2}, e^{3}\right\}$ independent of $t$. Thus in general the coframe "moves" as a function of $\underline{\xi}$. If $\beta$ is any form on spacetime it will be convenient to adopt the abbreviation $\dot{\beta}$ for $\mathcal{L}_{\frac{\partial}{\partial t}} \beta$, where $\mathcal{L}_{X}$ denotes the Lie derivative [7, [8], with respect to $X$. Thus $\dot{e}^{k}=0$ for $k=1,2,3$. Within this framework introduce the tensor fields:

$$
\underline{\hat{g}}=e^{1} \otimes e^{1}+e^{2} \otimes e^{2}, \quad \underline{g}=\underline{\hat{g}}+e^{3} \otimes e^{3}, \quad g=-e^{0} \otimes e^{0}+\underline{g},
$$

where $e^{0}=c_{0} d t$ and $g$ is the metric tensor field on Minkowski spacetime. At each instant $(t=$ constant $), \underline{g}$ is the induced metric tensor on Euclidean space and $\underline{\hat{g}}$ is the induced metric tensor on the 2-dimensional submanifolds (leaves) where $\xi_{3}=$ constant. Denote the Hodge map associated with $\underline{\hat{g}}$ by $\hat{\#}$ with

$$
\hat{\# 1}=e^{1} \wedge e^{2}
$$

and that associated with $\underline{g}$ by \# with

$$
\# 1=\hat{\#} 1 \wedge e^{3} .
$$

Then

$$
\star 1=\# 1 \wedge e^{0}:=e^{1} \wedge e^{2} \wedge e^{3} \wedge e^{0} .
$$


To accommodate the effects of signature it is convenient to introduce the involution operator $\eta$ on $p$-forms $\Phi$ by $\eta \Phi=(-1)^{p} \Phi$. Then

$$
\star \star=-\eta, \quad \# \#=1, \quad \hat{\#} \hat{\#}=\eta .
$$

By linearity the action of the Hodge map on an arbitrary form in Euclidean 3-space readily follows by expanding it in an orthonormal basis and using the relations

$$
\begin{aligned}
& \# e^{1}=e^{2} \wedge e^{3}, \\
& \# e^{2}=e^{3} \wedge e^{1}, \\
& \# e^{3}=e^{1} \wedge e^{2},
\end{aligned}
$$

on the basis forms. Furthermore in a 2-dimensional Euclidean space

$$
\begin{gathered}
\hat{\#} e^{1}=e^{2}, \\
\hat{\#} e^{2}=-e^{1} .
\end{gathered}
$$

If $\beta(\xi)$ is a $p$-form on spacetime but generated by forms in the exterior algebra generated by $\left\{e^{1}(\underline{\xi}), e^{2}(\underline{\xi}), e^{3}(\underline{\xi})\right\}$ then at any event with coordinates $\xi$ one has

$$
\underset{(p)}{\beta}(\xi)=\sum_{I} \beta_{I}(\xi) e^{I}(\underline{\xi}),
$$

where, for each multi-index $I$, the set of exterior $p$-forms $\left\{e^{I}(\underline{\xi})\right\}$ denotes a basis for $p$-forms generated from the set $\left\{e^{1}(\underline{\xi}), e^{2}(\underline{\xi}), e^{3}(\underline{\xi})\right\}$. One refers to the functions $\beta_{I}$ as the components of $\beta$ in the $e^{I}$ basis. With this notation $(p)$

$$
\underset{(p)}{\dot{\beta}}(\xi):=\sum_{I} \frac{\partial}{\partial \xi^{0}} \beta_{I}(\xi) e^{I}(\underline{\xi}) .
$$

Define the $2+1$ split of $\underset{(p)}{\beta}(\xi)$ into the pair $\underset{(p-1)}{\{\hat{\beta}}(\xi), \underset{(p)}{\hat{\beta}}(\xi)\}$ by the unique decomposition with respect to $\boldsymbol{d} \xi^{3}$ :

$$
\underset{(p)}{\beta}(\xi)=\underset{(p-1)}{\hat{\beta}}(\xi) \wedge \boldsymbol{d} \xi^{3}+\underset{(p)}{\hat{\beta}}(\xi),
$$

where $\underset{(p-1)}{\hat{\beta}}(\xi)$ and $\underset{(p)}{\hat{\beta}}(\xi)$ are $p-1$ and $p$-forms respectively, generated from the 1 -forms in $\left\{\boldsymbol{d} \xi^{1}, \boldsymbol{d} \xi^{2}\right\}$ satisfying $i_{\frac{\partial}{\partial \xi^{3}}} \hat{\beta}(p-1)(\xi)=0$ and $i_{\frac{\partial}{\partial \xi^{3}}} \hat{\beta}(p)=0$. Thus $\underset{(p-1)}{\hat{\beta}}$ and $\underset{(p)}{\hat{\beta}}$ are forms that do not contain $\boldsymbol{d} \xi^{3}$.

It follows that for $q=0,1,2$ :

$$
\begin{aligned}
& \left.\# \underset{(q)}{(\hat{\beta}} \wedge e^{3}\right)=\underset{(q)}{\#}(\eta \underset{\beta}{\hat{\beta}}), \\
& \# \underset{(q)}{(\hat{\beta})}=\underset{(q)}{\hat{\beta}}(\underset{\beta}{\hat{\beta}}) \wedge e^{3} .
\end{aligned}
$$

For any 0 -form $\hat{\beta}$

$$
\underset{(0)}{\boldsymbol{d}} \hat{\beta}=\underset{(0)}{\hat{\boldsymbol{d}} \hat{\beta}}+\left(\mathcal{L}_{\frac{\partial}{\partial \xi^{3}}} \underset{(0)}{\hat{\beta}}\right) \boldsymbol{d} \xi^{3},
$$


where

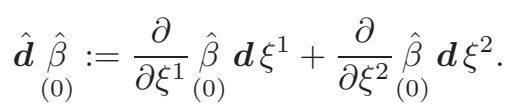

From this it follows that, for $q=0,1,2$ :

$$
\underset{(q)}{\boldsymbol{d}} \hat{\beta}=\underset{(q)}{\hat{\boldsymbol{d}} \hat{\beta}}+\boldsymbol{d} \xi^{3} \wedge\left(\mathcal{L}_{\frac{\partial}{\partial \xi^{3}}} \underset{(q)}{\hat{\beta}}\right)
$$

where $\hat{\boldsymbol{d}}$ acts [17] on exterior forms generated by $\left\{\boldsymbol{d} \xi^{1}, \boldsymbol{d} \xi^{2}\right\}$. Note that for all 2-forms $\hat{\beta}$ one has $\hat{\boldsymbol{d}} \hat{\beta}=0$. Let the $3+1$ split of the 4 -current 3 -form be

$$
\underset{(3)}{j}(\xi)=-\underset{(2)}{J}(\xi) \wedge d t+\underset{(0)}{\rho}(\xi) \# 1
$$

with $i_{\frac{\partial}{\partial t}} J=0$. Then, from (7)

$$
d j=0
$$

yields

$$
\boldsymbol{d} \underset{(2)}{J}(\xi)+\underset{(0)}{\dot{\rho}}(\xi) \# 1=0 .
$$

It is convenient to introduce the (Hodge) dual forms:

$$
\underset{(2)}{\mathbf{E}}:=\# \underset{(1)}{\mathbf{e}}, \quad \underset{(2)}{\mathbf{D}}:=\#_{(1)}^{\mathbf{d}}, \quad \underset{(2)}{\mathbf{B}}:=\#_{(1)}^{\mathbf{b}}, \quad \underset{(2)}{\mathbf{H}}:=\#_{(1)}^{\mathbf{h}},
$$

so that the $3+1$ split of the spacetime covariant Maxwell equations (7) with respect to $d t$ becomes

$$
\begin{gathered}
\boldsymbol{d}_{(1)}^{\mathbf{e}}=-\underset{(2)}{\dot{\mathbf{B}}}, \\
\boldsymbol{d} \underset{(2)}{\mathbf{B}}=0, \\
\boldsymbol{d} \underset{(1)}{\mathbf{h}}=\underset{(2)}{J}+\underset{(2)}{\dot{\mathbf{D}}}, \\
\boldsymbol{d} \underset{(2)}{\mathbf{D}}=\underset{(0)}{\rho \# 1} .
\end{gathered}
$$

All $p$-forms $(p \geq 1)$ in these equations are independent of $e^{0}$ but may depend on $t$. Furthermore they are independent of the choice of (stationary) spatial co-frame constructed from $\left\{\boldsymbol{d} \xi^{1}, \boldsymbol{d} \xi^{2}, \boldsymbol{d} \xi^{3}\right\}$, in any chart with local coordinates $\xi^{1}, \xi^{2}, \xi^{3}$.

In the following it is assumed that $\underset{(1)}{\mathbf{b}}=\mu \underset{(1)}{\mathbf{h}}$ and $\underset{(1)}{\mathbf{d}}=\varepsilon \underset{(1)}{\mathbf{e}}$ (with constant $\varepsilon, \mu$ ) where $\varepsilon=\epsilon_{r} \epsilon_{0}, \mu=\mu_{r} \mu_{0}$. Thus in terms of $\underset{(1)}{\mathbf{e}} \underset{(1)}{\mathbf{h}} \underset{(2)}{\mathbf{E}}, \underset{(2)}{\mathbf{H}}$ :

$$
\begin{gathered}
\underset{(1)}{\boldsymbol{d}}=-\mu \underset{(2)}{\dot{\mathbf{H}}}, \\
\boldsymbol{d} \underset{(2)}{\mathbf{H}}=0, \\
\boldsymbol{d} \underset{(1)}{\mathbf{h}}=\varepsilon \underset{(2)}{\dot{\mathbf{E}}}+\underset{(2)}{J}, \\
\varepsilon \boldsymbol{d} \underset{(2)}{\mathbf{E}}=\rho \# 1 .
\end{gathered}
$$




\section{THE MAXWELL SYSTEM WITH SOURCES IN A CURVED BEAM PIPE}

In terms of time dependent 1-form electromagnetic fields with general sources $(\rho, J)$ the Maxwell's equations in a medium with scalar permeability $\mu$ can be written

(0) (2)

$$
\begin{aligned}
& \boldsymbol{d} \underset{(1)}{\mathbf{e}}+\mu \# \underset{(1)}{\mathbf{h}}=0 \\
& \boldsymbol{d} \underset{(1)}{\mathbf{h}}-\mu Y^{2} \# \underset{(1)}{\dot{\mathbf{e}}}-\underset{(2)}{J}=0, \\
& \boldsymbol{d} \# \underset{(1)}{\mathbf{h}}=0 \\
& \mu Y^{2} \boldsymbol{d} \# \underset{(1)}{\mathbf{e}}-\underset{(0)}{\rho} \# 1=0
\end{aligned}
$$

where the admittance $Y=1 /(\mu c)$ with $c$ being the speed of light in the medium.

These equations involve time dependent forms and are independent of particular local spatial coordinates. They depend explicitly on the Euclidean metric and for conserved sources define a well posed initial-boundary value problem. We now choose a coordinate system adapted to the interior $\mathcal{U}$ of a beam pipe with a circular disc cross-section of fixed radius $a$ at every point and an axis given by a planar space-curve with, in general, non-constant curvature $\kappa$ and $|\kappa a| \ll 1$. At each point on this curve one may erect a triad of orthogonal vectors in space, one member of which is tangent to the curve. The remaining vectors define a transverse plane. All points in the interior $\mathcal{U}$ of the beam pipe lie on some transverse plane associated with such a triad with origin at some point on the axial space-curve. Let the region $\mathcal{U} \subset \mathbb{R}^{3}$ inside the beam pipe be described in terms of coordinates $\left(\xi^{1}, \xi^{2}, \xi^{3}\right):=(r, \theta, z)$ adapted to the central space-curve with curvature $\kappa(z)$ such that

$$
0 \leq r \leq a, \quad 0<\theta \leq 2 \pi, \quad-\infty \leq z \leq \infty .
$$

A convenient field of orthonormal coframes [9] on $\mathcal{U}$ is given in these coordinates by

$$
\left\{e^{1}=\boldsymbol{d} r, \quad e^{2}=r \boldsymbol{d} \theta, \quad e^{3}=\left(1-\epsilon \kappa_{0}(z) x_{1}\right) \boldsymbol{d} z\right\},
$$

with $x_{1}=r \cos \theta$. Thus the Euclidean metric tensor $\underline{g}$ on $\mathcal{U}$ is given by

$$
\underline{g}=e^{1} \otimes e^{1}+e^{2} \otimes e^{2}+e^{3} \otimes e^{3} .
$$

In these coordinates the pipe boundary is the surface $r=a$, the coordinate $z$ measures arc-length along the space-curve and on the space-curve $r=0$.

It proves convenient in the following to write $\kappa(z)=\epsilon \kappa_{0}(z)$ and use $\epsilon$ as a book-keeping device in order to keep track of different orders of $\kappa$. In terms of adapted coordinates

$$
\underline{g}=\underline{\hat{g}}+\left(1-\epsilon \kappa_{0}(z) x_{1}\right)^{2} \boldsymbol{d} z \otimes \boldsymbol{d} z,
$$

where for each cross-section at constant $z$ one has the induced metric tensor $\underline{g}$ on the 2 -dimensional disc $(0 \leq r \leq$ $a, 0<\theta \leq 2 \pi)$ :

$$
\underline{\hat{g}}=\boldsymbol{d} r \otimes \boldsymbol{d} r+r^{2} \boldsymbol{d} \theta \otimes \boldsymbol{d} \theta
$$

The associated contravariant tensors are

$$
\begin{aligned}
& \underline{\hat{g}}^{-1}=\frac{\partial}{\partial r} \otimes \frac{\partial}{\partial r}+\frac{1}{r^{2}} \frac{\partial}{\partial \theta} \otimes \frac{\partial}{\partial \theta} \\
& \underline{g}^{-1}=\underline{\hat{g}}^{-1}+\frac{1}{\left(1-\epsilon \kappa_{0}(z) x_{1}\right)^{2}} \frac{\partial}{\partial z} \otimes \frac{\partial}{\partial z} .
\end{aligned}
$$

The source forms will be expressed in terms of the scalar functions $J_{r}, J_{\theta}, J_{0}$ and $\rho$ of $(\epsilon, t, z, r, \theta)$. We choose to write $J$ as

$(2)$

$$
\begin{aligned}
& \underset{(2)}{J}(\epsilon, t, z, r, \theta) \\
& =\left(J_{\theta}(\epsilon, t, z, r, \theta) \boldsymbol{d} r+r J_{r}(\epsilon, t, z, r, \theta) \boldsymbol{d} \theta\right) \wedge \boldsymbol{d} z\left(1-\epsilon \kappa_{0}(z) r \cos \theta\right) \\
& \quad+J_{0}(\epsilon, t, z, r, \theta) r \boldsymbol{d} r \wedge \boldsymbol{d} \theta,
\end{aligned}
$$


from which it immediately follows that

$$
\# \underset{(2)}{J}=-J_{\theta} r \boldsymbol{d} \theta+J_{r} \boldsymbol{d} r+J_{0}\left(1-\epsilon \kappa_{0}(z) x_{1}\right) \boldsymbol{d} z
$$

with orthogonal components $\left(-J_{\theta}, J_{r}, J_{0}\right)$ of $\# \underset{(2)}{J}$. The associated electric current vector field is $\widetilde{\#} \underset{(2)}{J}$.

The objective is to solve (26), (27), (28), (29) for the fields $\underset{(1)}{\mathbf{e}}$ and $\underset{(1)}{\mathbf{h}}$ on $\mathcal{U}$ in terms of prescribed sources and initial data as a perturbative expansion in the axial curvature of the beam pipe. The strategy will be to project the field system into suitable modes that ensure that perfectly conducting boundary conditions are satisfied at the pipe boundary. In the adapted coordinate system this is achieved with the aid of complex Dirichelet and Neumann eigen-modes of the two-dimensional Laplacian associated with each transverse plane in the beam pipe.

\section{A. Dirichelet Modes}

Let $\mathcal{D}$ be the smooth 2-dimensional submanifold ( $z=$ constant) with boundary $\partial \mathcal{D}$, embedded in Eucldean $\mathbb{R}^{3}$. The tensor $\underline{\hat{g}}$ on $\mathcal{D}$ is that induced from the Euclidean metric $\underline{g}$ in $\mathbb{R}^{3}$. A complex Dirichelet mode set $\left\{\Phi_{N}\right\}$ is a collection of complex eigen 0 -forms of the Laplacian operator $-\boldsymbol{d} \hat{\#} \boldsymbol{d}$ on $\mathcal{D}$ (associated with the metric $\hat{g}$ and Hodge operator $\hat{\#}$ ) that vanishes on $\partial \mathcal{D}$. This boundary condition and the nature of the domain determine the associated (positive non-zero real) eigenvalues $\beta_{N}^{2}$. The label $N$ here consists of an ordered pair of real numbers. Thus

$$
\Phi_{N}: \mathcal{D} \rightarrow \mathbb{R}, \quad r, \theta \mapsto \Phi_{N}(r, \theta),
$$

satisfies

$$
\boldsymbol{d} \hat{\#} \boldsymbol{d} \Phi_{N}+\beta_{N}^{2} \Phi_{N} \hat{\#} 1=0
$$

with $\left.\Phi_{N}\right|_{\partial \mathcal{D}}=0$. It is straightforward to show from these properties that if $\beta_{N}^{2} \neq \beta_{M}^{2} \neq 0,(N \neq M)$ then

$$
\int_{\mathcal{D}} \overline{\Phi_{M}} \Phi_{N} \hat{\# 1}=0
$$

where the bar denotes complex conjugation. If one normalises these modes so that

$$
\int_{\mathcal{D}} \overline{\Phi_{M}} \Phi_{N} \hat{\#} 1=\mathcal{N}_{N}^{2} \delta_{N M}
$$

then it is also easy to show that

$$
\int_{\mathcal{D}} \boldsymbol{d} \overline{\Phi_{N}} \wedge \hat{\#} \boldsymbol{d} \Phi_{M}=\beta_{N}^{2} \mathcal{N}_{N}^{2} \delta_{N M}
$$

An explicit form for $\Phi_{N}$ is for $n \in \mathbb{Z}$

$$
\Phi_{N}(r, \theta)=J_{n}\left(x_{q(n)} \frac{r}{a}\right) e^{i n \theta},
$$

where $J_{n}(x)$ is the $n$-th Bessel function and the numbers $\left\{x_{q(n)}\right\}$ are defined by $J_{n}\left(x_{q(n)}\right)=0$ and $N:=\{n, q(n)\}$. The eigenvalues are given by $\left\{\beta_{N}=x_{q(n)} / a\right\}$. It follows from the integral [10]

$$
\int_{0}^{a} d r r J_{m}\left(x_{q(m)} \frac{r}{a}\right) J_{m}\left(x_{q^{\prime}(m)} \frac{r}{a}\right)=\frac{a^{2}}{2} J_{m+1}^{2}\left(x_{q(m)}\right) \delta_{q(m), q^{\prime}(m)},
$$

that $\mathcal{N}_{N}^{2}=\pi a^{2} J_{n+1}^{2}\left(x_{q(n)}\right)$. 


\section{B. Neumann Modes}

In a similar manner one defines a Neumann mode set $\left\{\Psi_{N}\right\}$ as a collection of eigen 0-forms of the Laplacian operator on $\mathcal{D}$ such that $\hat{\#} \boldsymbol{d} \Psi_{N}$ vanishes on $\partial \mathcal{D}$. This alternative boundary condition and the nature of the domain determine the associated (positive non-zero real) eigenvalues $\alpha_{N}^{2}$ where again the label $N$ consists of an ordered pair of real numbers. Thus

$$
\Psi_{N}: \mathcal{D} \rightarrow \mathbb{R}, \quad r, \theta \mapsto \Psi_{N}(r, \theta),
$$

satisfies

$$
\boldsymbol{d} \hat{\#} \boldsymbol{d} \Psi_{N}+\alpha_{N}^{2} \Psi_{N} \hat{\#} 1=0,
$$

with $\left.\hat{\#} \boldsymbol{d} \Psi_{N}\right|_{\partial \mathcal{D}}=0$ It is straightforward to show from these properties that if $\alpha_{N}^{2} \neq \alpha_{M}^{2} \neq 0,(N \neq M)$ then

$$
\int_{\mathcal{D}} \overline{\Psi_{M}} \Psi_{N} \hat{\#} 1=0
$$

If one normalises these modes so that

$$
\int_{\mathcal{D}} \overline{\Psi_{M}} \Psi_{N} \hat{\#} 1=\mathcal{M}_{N}^{2} \delta_{N M}
$$

then it is also easy to show that

$$
\int_{\mathcal{D}} \boldsymbol{d} \overline{\Psi_{N}} \wedge \hat{\#} \boldsymbol{d} \Psi_{M}=\alpha_{N}^{2} \mathcal{M}_{N}^{2} \delta_{N M}
$$

An explicit form for $\Psi_{M}$ is for $m \in \mathbb{Z}$

$$
\Psi_{M}(r, \theta)=J_{m}\left(x_{p(m)}^{\prime} \frac{r}{a}\right) e^{i m \theta},
$$

where the numbers $\left\{x_{p(m)}^{\prime}\right\}$ are defined by $J_{m}^{\prime}\left(x_{p(m)}^{\prime}\right)=0$ and $M:=\{m, p(m)\}$. The eigenvalues are given by $\left\{\alpha_{M}=x_{p(m)}^{\prime} / a\right\}$ and $\mathcal{M}_{M}^{2}=\pi a^{2} J_{m+1}^{2}\left(x_{p(m)}^{\prime}\right)$.

\section{Mode Decompositions}

Since $\mathcal{U}$ is simply connected one can represent the electromagnetic 1 -forms $\underset{(1)}{\mathbf{e}}=\underset{(1)}{\mathbf{e}}(\epsilon, t, z, r, \theta)$ and $\underset{(1)}{\mathbf{h}}=$ $\underset{(1)}{\mathbf{h}}(\epsilon, t, z, r, \theta)$ as [1]

$$
\begin{aligned}
& \underset{(1)}{\mathbf{e}}(\epsilon, t, z, r, \theta)=\sum_{N} V_{N}^{E}(\epsilon, t, z) \boldsymbol{d} \Phi_{N}+\sum_{M} V_{M}^{H}(\epsilon, t, z) \#\left(\boldsymbol{d} z \wedge \boldsymbol{d} \Psi_{M}\right) \\
& +\sum_{N} \gamma_{N}^{E}(\epsilon, t, z) \Phi_{N}(r, \theta) \boldsymbol{d} z, \\
& \underset{(1)}{\mathbf{h}}(\epsilon, t, z, r, \theta)=\sum_{N} I_{N}^{E}(\epsilon, t, z) \#\left(\boldsymbol{d} z \wedge \boldsymbol{d} \Phi_{N}\right)+\sum_{M} I_{M}^{H}(\epsilon, t, z) \boldsymbol{d} \Psi_{M} \\
& +\sum_{M} \gamma_{M}^{H}(\epsilon, t, z) \Psi_{M}(r, \theta) \boldsymbol{d} z .
\end{aligned}
$$

Here, for any scalars $f_{M}$ with $M=(m, p(m))$ and $f_{N}$ with $N=(n, q(n))$, the summations above are abbreviations for:

$$
\sum_{M} f_{M} \cdots=\sum_{m \in \mathbb{Z}} \sum_{p(m) \in \mathbb{N}} f_{m, p(m)} \cdots, \quad \sum_{N} f_{N} \cdots=\sum_{n \in \mathbb{Z}} \sum_{q(n) \in \mathbb{N}} f_{n, q(n)} \cdots
$$

For future convenience the further abbreviation:

$$
\sum_{N}^{\prime} f_{N} \cdots=\sum_{n=m \pm 1} \sum_{q(n) \in \mathbb{N}} f_{n, q(n)} \cdots
$$


will be used. The expansions above in terms of $\Phi_{N}$ and $\Psi_{M}$ and their derivatives ensure that the electromagnetic fields satisfy perfectly conducting boundary conditions at the surface $r=a$.

Since $\left.\Phi\right|_{r=a}=0$ we note that

$$
\int_{\mathcal{D}} \boldsymbol{d} \Psi_{M} \wedge \boldsymbol{d} \Phi_{N}=\int_{\partial \mathcal{D}} \Phi_{N} \boldsymbol{d} \Psi_{M}=0
$$

and for $m, n \in \mathbb{Z}$

$$
\delta_{m, n}=\int_{0}^{2 \pi} \frac{d \theta}{2 \pi} e^{i(m-n) \theta}
$$

Furthermore with $n \in \mathbb{Z}$,

$$
J_{-n}(x)=(-1)^{n} J_{n}(x)
$$

so

$$
p(m)=p(-m), \quad \text { and } \quad q(n)=q(-n) .
$$

Hence

$$
\alpha_{n, q(n)}=\alpha_{-n, q(-n)}, \quad \beta_{n, q(n)}=\beta_{-n, q(-n)},
$$

and

$$
\boldsymbol{d} \Phi_{n, q(n)}=(-1)^{n} \boldsymbol{d} \overline{\Phi_{-n, q(-n)}}, \quad \boldsymbol{d} \Psi_{m, p(m)}=(-1)^{m} \boldsymbol{d} \overline{\Psi_{-m, p(-m)}} .
$$

From Eq. (43) and the reality conditions,

$$
\underset{(1)}{\mathbf{e}}=\overline{(1)}, \quad \text { and } \quad \underset{(1)}{\mathbf{e}}=\overline{(1)},
$$

one has

$$
\begin{aligned}
& V_{n, q(n)}^{E}=(-1)^{n} \overline{V_{-n, q(-n)}^{E}}, V_{m, p(m)}^{H}=(-1)^{m} \overline{V_{-m, q(-m)}^{H}}, \\
& \gamma_{n, q(n)}^{E}=(-1)^{n} \overline{\gamma_{-n, q(-n)}^{E}}, \\
& I_{n, q(n)}^{E}=(-1)^{n} \overline{I_{-n, q(-n)}^{E}}, I_{m, p(m)}^{H}=(-1)^{m} \overline{I_{-m, q(-m)}^{H}}, \\
& \gamma_{m, p(m)}^{H}=(-1)^{m} \overline{\gamma_{-m, p(-m)}^{H}} .
\end{aligned}
$$

Similarly from the relations

$$
z J_{n}^{\prime}(z)=n J_{n}(z)-z J_{n+1}(z)
$$

it follows that

$$
\mathcal{N}_{-n, q(-n)}^{2}=\mathcal{N}_{n, q(n)}^{2}, \quad \text { and } \quad \mathcal{M}_{-m, p(-m)}^{2}=\mathcal{M}_{m, p(m)}^{2} .
$$

These relations enable one to pass simply from complex to real representations of the mode summations for the fields above.

\section{Perturbation Expansions}

Since for small $|\kappa a|$ the beam pipe approximates a straight cylinder we adopt the perturbative field-mode expansions

$$
\begin{gathered}
V_{N}^{E}(\epsilon, t, z)=V_{N}^{E(0)}(t, z)+\epsilon V_{N}^{E(1)}(t, z)+\mathcal{O}\left(\epsilon^{2}\right) . \\
I_{N}^{E}(\epsilon, t, z)=I_{N}^{E(0)}(t, z)+\epsilon I_{N}^{E(1)}(t, z)+\mathcal{O}\left(\epsilon^{2}\right) .
\end{gathered}
$$




$$
\gamma_{N}^{E}(\epsilon, t, z)=\gamma_{N}^{E(0)}(t, z)+\epsilon \gamma_{N}^{E(1)}(t, z)+\mathcal{O}\left(\epsilon^{2}\right),
$$

with analogous expansions for the magnetic modes $V_{M}^{H}, I_{M}^{H}, \gamma_{M}^{H}$ and express the sources as a power series in $\epsilon$ :

$$
\begin{aligned}
J_{\theta}(\epsilon, t, z, r, \theta) & =J_{\theta}^{(0)}(t, z, r, \theta)+\epsilon J_{\theta}^{(1)}(t, z, r, \theta)+\mathcal{O}\left(\epsilon^{2}\right), \\
J_{r}(\epsilon, t, z, r, \theta) & =J_{r}^{(0)}(t, z, r, \theta)+\epsilon J_{r}^{(1)}(t, z, r, \theta)+\mathcal{O}\left(\epsilon^{2}\right), \\
J_{0}(\epsilon, t, z, r, \theta) & =J_{0}^{(0)}(t, z, r, \theta)+\epsilon J_{0}^{(1)}(t, z, r, \theta)+\mathcal{O}\left(\epsilon^{2}\right), \\
\rho(\epsilon, t, z, r, \theta) & =\rho^{(0)}(t, z, r, \theta)+\epsilon \rho^{(1)}(t, z, r, \theta)+\mathcal{O}\left(\epsilon^{2}\right) .
\end{aligned}
$$

These expansions are then inserted into (26), (27), (28), (29) and $\mathcal{O}\left(\epsilon^{n}\right)$ systems extracted for $n=0,1$. For general sources it is somewhat tedious to project out the equations for the perturbative field coefficients above. This is achieved using the orthogonality relations between the different Dirichelet and Neumann mode sets and the explicit relations listed in Appendix A to integrate (26), (27), (28), (29) over the domain $\mathcal{D}$.

In order to express the resulting equations for the mode amplitudes in a unified way we define

$$
\Xi_{M, N}^{k}(\mathcal{F}, \mathcal{G}, x, y):=\int_{0}^{a} d r r^{k} \mathcal{F}_{m}\left(x_{p(m)} \frac{r}{a}\right) \mathcal{G}_{n}\left(y_{q(n)} \frac{r}{a}\right)
$$

and note

$$
\Xi_{M, N}^{k}(\mathcal{F}, \mathcal{F}, x, x)=\Xi_{N, M}^{k}(\mathcal{F}, \mathcal{F}, x, x) .
$$

Furthermore if $\mathcal{F}$ is a Bessel function $J$ or its derivative, $J^{\prime}$ :

$$
\Xi_{M, N}^{k}(\mathcal{F}, \mathcal{F}, x, x)=(-1)^{m+n} \Xi_{-m, p(-m),-n, q(-n)}^{k}(\mathcal{F}, \mathcal{F}, x, x) .
$$

Thus

$$
\begin{aligned}
\int_{0}^{a} d r r J_{m}\left(x_{p(m)}^{\prime} \frac{r}{a}\right) J_{n}^{\prime}\left(x_{q(n)}^{\prime} \frac{r}{a}\right) & =\Xi_{M, N}^{1}\left(J, J^{\prime}, x^{\prime}, x^{\prime}\right), \\
\int_{0}^{a} d r J_{m}\left(x_{p(m)}^{\prime} \frac{r}{a}\right) J_{n}\left(x_{q(n)}^{\prime} \frac{r}{a}\right) & =\Xi_{M, N}^{0}\left(J, J, x^{\prime}, x^{\prime}\right) .
\end{aligned}
$$

The symbol $\Xi_{M, N}^{k}(\mathcal{F}, \mathcal{G}, x, y)$ enables one to write more compactly certain overlap coefficients that arise in the projections of (26), (27), (28), (29) over $\mathcal{D}$. These coefficients are given in Appendix A.

Using these definitions the projection of (26) to lowest order $\mathcal{O}\left(\epsilon^{0}\right)$ is

$$
\begin{aligned}
& V_{M}^{E(0) \prime}+\mu \dot{I}_{M}^{E(0)}-\gamma_{M}^{E(0)}=0, \\
& V_{M}^{H(0) \prime}-\mu \dot{I}_{M}^{H(0)}=0, \\
& \mu \dot{\gamma}_{M}^{H(0)}-\alpha_{M}^{2} V_{M}^{H(0)}=0,
\end{aligned}
$$

and to first order $\mathcal{O}\left(\epsilon^{1}\right)$ is

$$
\begin{array}{r}
\left(V_{M}^{E(1) \prime}+\mu \dot{I}_{M}^{E(1)}-\gamma_{M}^{E(1)}\right) \beta_{M}^{2} \mathcal{N}_{M}^{2} \\
-\kappa_{0}(z) \sum_{N}^{\prime}\left(V_{N}^{H(0) \prime}+\mu \dot{I}_{N}^{H(0)}\right) G_{M, N}^{\bar{\Phi}, \Psi}-\kappa_{0}^{\prime}(z) \sum_{N}^{\prime} V_{N}^{H(0)} G_{M, N}^{\bar{\Phi}, \Psi}=0, \\
\left(V_{M}^{H(1) \prime}-\mu \dot{I}_{M}^{H(1)}\right) \alpha_{M}^{2} \mathcal{M}_{M}^{2}+\kappa_{0}(z) \sum_{N}^{\prime}\left(V_{N}^{H(0) \prime}+\mu \dot{I}_{N}^{H(0)}\right) F_{M, N}^{\Phi} \\
+\kappa_{0}^{\prime}(z) \sum_{N}^{\prime} V_{N}^{H(0)} F_{M, N}^{\Phi}=0, \\
\left(\mu \dot{\gamma}_{M}^{H(1)}-\alpha_{M}^{2} V_{M}^{H(1)}\right) \mathcal{M}_{M}^{2}+\kappa_{0}(z) \sum_{N}^{\prime}\left(\mu \dot{\gamma}_{N}^{H(0)}-\alpha_{N}^{2} V_{N}^{H(0)}\right) E_{M, N}^{\Psi} \\
+\kappa_{0}(z) \sum_{N}^{\prime} V_{N}^{H(0)}\left(C_{M, N}^{\Psi}-D_{M, N}^{\Psi}\right)=0 .
\end{array}
$$


where a superscript prime attached to a function denotes its partial derivative with respect to $z$ and all non-constant functions in (54),(55), (56) depend on $z$ or $z, t$.

The projection of (27) to lowest order $\mathcal{O}\left(\epsilon^{0}\right)$ is

$$
\begin{array}{r}
-\left(I_{M}^{H(0) \prime}-\mu Y^{2} \dot{V}_{M}^{H(0)}-\gamma_{M}^{H(0)}\right) \alpha_{M}^{2} \mathcal{M}_{M}^{2} \\
+\int_{\mathcal{D}} J_{\theta}^{(0)}\left(\hat{\#} \boldsymbol{d} \overline{\Psi_{M}}\right) \wedge \boldsymbol{d} r+\int_{\mathcal{D}} r J_{r}^{(0)}\left(\hat{\#} \boldsymbol{d} \overline{\Psi_{M}}\right) \wedge \boldsymbol{d} \theta=0 \\
\left(I_{M}^{E(0) \prime}+\mu Y^{2} \dot{V}_{M}^{E(0)}\right) \beta_{M}^{2} \mathcal{N}_{M}^{2} \\
+\int_{\mathcal{D}} J_{\theta}^{(0)} \boldsymbol{d} \overline{\Phi_{M}} \wedge \boldsymbol{d} r+\int_{\mathcal{D}} r J_{r}^{(0)} \boldsymbol{d} \overline{\Phi_{M}} \wedge \boldsymbol{d} \theta=0 \\
\left(-\beta_{M}^{2} I_{M}^{E(0)}-\mu Y^{2} \dot{\gamma}_{M}^{E(0)}\right) \mathcal{N}_{M}^{2}-\int_{\mathcal{D}} J_{0}^{(0)} \overline{\Phi_{M}} \hat{\#} 1=0 .
\end{array}
$$

and to first order $\mathcal{O}\left(\epsilon^{1}\right)$ is

$$
\begin{array}{r}
-\left(I_{M}^{H(1) \prime}-\mu Y^{2} \dot{V}_{M}^{H(1)}-\gamma_{M}^{H(1)}\right) \alpha_{M}^{2} \mathcal{M}_{M}^{2} \\
+\kappa_{0}(z) \sum_{N}^{\prime}\left(I_{N}^{E(0) \prime}-\mu Y^{2} \dot{V}_{N}^{E(0)}\right) G_{M, N}^{\bar{\Psi}, \Phi} \\
+\kappa_{0}^{\prime}(z) \sum_{N}^{\prime} I_{N}^{E(0)} G_{M, N}^{\bar{\Psi}, \Phi}+\int_{\mathcal{D}}\left(J_{\theta}^{(1)}-J_{\theta}^{(0)} \kappa_{0}(z) r \cos \theta\right)\left(\hat{\#} \boldsymbol{d} \overline{\Psi_{M}}\right) \wedge \boldsymbol{d} r \\
+\int_{\mathcal{D}} r\left(J_{r}^{(1)}-J_{r}^{(0)} \kappa_{0}(z) r \cos \theta\right)\left(\hat{\#} \boldsymbol{d} \overline{\Psi_{M}}\right) \wedge \boldsymbol{d} \theta=0, \\
\left(I_{M}^{E(1) \prime}+\mu Y^{2} \dot{V}_{M}^{E(1)}\right) \beta_{M}^{2} \mathcal{N}_{M}^{2}+\kappa_{0}(z) \sum_{N}^{\prime}\left(I_{N}^{E(0) \prime}-\mu Y^{2} \dot{V}_{N}^{E(0)}\right) F_{M, N}^{\Phi} \\
+\kappa_{0}^{\prime}(z) \sum_{N}^{\prime} I_{N}^{E(0)} F_{M, N}^{\Phi}+\int_{\mathcal{D}}\left(J_{\theta}^{(1)}-J_{\theta}^{(0)} \kappa_{0}(z) r \cos \theta\right) \boldsymbol{d} \overline{\Phi_{M}} \wedge \boldsymbol{d} r \\
+\int_{\mathcal{D}} r\left(J_{r}^{(1)}-J_{r}^{(0)} \kappa_{0}(z) r \cos \theta\right) \boldsymbol{d} \overline{\Phi_{M}} \wedge \boldsymbol{d} \theta=0, \\
-\left(I_{M}^{E(1)} \beta_{M}^{2}+\mu Y^{2} \dot{\gamma}_{M}^{E(1)}\right) \mathcal{N}_{M}^{2}-\kappa_{0}(z) \sum_{N}^{\prime}\left(\beta_{N}^{2} I_{N}^{E(0)}+\mu Y^{2} \dot{\gamma}_{N}^{E(0)}\right) E_{M, N}^{\Phi} \\
+\kappa_{0}(z) \sum_{N}^{\prime} I_{N}^{E(0)}\left(C_{M, N}^{\Phi}-D_{M, N}^{\Phi}\right)-\int_{\mathcal{D}} J_{0}^{(1)} \overline{\Phi_{M}} \hat{\#} 1=0 .
\end{array}
$$

The projection of (28) to lowest order $\mathcal{O}\left(\epsilon^{0}\right)$ is

$$
\gamma_{M}^{H(0) \prime}-\alpha_{M}^{2} I_{M}^{H(0)}=0 .
$$

and to first order $\mathcal{O}\left(\epsilon^{1}\right)$,

$$
\begin{array}{r}
\left(\gamma_{M}^{H(1) \prime}-\alpha_{M}^{2} I_{M}^{H(1)}\right) \mathcal{M}_{M}^{2}+\kappa_{0}(z) \sum_{N}^{\prime}\left(\gamma_{N}^{H(0) \prime}+\alpha_{N}^{2} I_{N}^{H(0)}\right) E_{M, N}^{\Psi} \\
+\kappa_{0}(z) \sum_{N}^{\prime} I_{N}^{H(0)}\left(-C_{M, N}^{\Psi}+D_{M, N}^{\Psi}\right)=0 .
\end{array}
$$

Finally the projection of (29) to lowest order $\mathcal{O}\left(\epsilon^{0}\right)$ is

$$
\mu Y^{2}\left(\gamma_{M}^{E(0) \prime}-\beta_{M}^{2} V_{M}^{E(0)}\right) \mathcal{N}_{M}^{2}-\int_{\mathcal{D}} \rho^{(0)} \overline{\Phi_{M}} \hat{\#} 1=0 .
$$


and to first order $\mathcal{O}\left(\epsilon^{1}\right)$,

$$
\begin{array}{r}
\mu Y^{2}\left(\gamma_{M}^{E(1) \prime}-\beta_{M}^{2} V_{M}^{E(1)}\right) \mathcal{N}_{M}^{2}-\int_{\mathcal{D}}\left(\rho^{(1)}-\rho^{(0)} \kappa_{0}(z) r \cos \theta\right) \overline{\Phi_{M}} \hat{\#} 1 \\
+\mu Y^{2} \kappa_{0}(z) \sum_{N}^{\prime}\left\{\left(V_{N}^{E(0)} \beta_{N}^{2}+\gamma_{N}^{E(0) \prime}\right) E_{M, N}^{\Phi}+V_{N}^{E(0)}\left(-C_{M, N}^{\Phi}+D_{M, N}^{\Phi}\right)\right\}=0 .
\end{array}
$$

\section{Decoupling to lowest order}

The equations above to lowest order describe the fields that can be excited by sources in a perfectly conducting straight beam pipe. They are readily decoupled by substituting (53) and (63) into (57) yielding an inhomogeneous telegraph-type equation for $\gamma_{N}^{H(0)}$ :

$$
\begin{aligned}
& \ddot{\gamma}_{N}^{H(0)}-c^{2} \gamma_{N}^{H(0) \prime \prime}+c^{2} \alpha_{N}^{2} \gamma_{N}^{H(0)} \\
& =\frac{-c^{2}}{\mathcal{M}_{N}^{2}}\left\{\int_{\mathcal{D}} J_{\theta}^{(0)}\left(\hat{\#} \overline{\Psi_{N}}\right) \wedge \boldsymbol{d} r+\int_{\mathcal{D}} r J_{r}^{(0)}\left(\hat{\#} \overline{\Psi_{N}}\right) \wedge \boldsymbol{d} \theta\right\} .
\end{aligned}
$$

Similarly the equation for $\gamma_{N}^{E(0)}$ follows by substituting (59) and (65) into (51):

$$
\begin{aligned}
& \ddot{\gamma}_{N}^{E(0)}-c^{2} \gamma_{N}^{E(0) \prime \prime}+c^{2} \beta_{N}^{2} \gamma_{N}^{E(0)} \\
& =-\frac{1}{\mu Y^{2} \mathcal{N}_{N}^{2}} \frac{\partial}{\partial t}\left(\int_{\mathcal{D}} J_{0}^{(0)} \overline{\Phi_{N}} \hat{\#} 1\right)-\frac{c^{4} \mu}{\mathcal{N}_{N}^{2}} \frac{\partial}{\partial z}\left(\int_{\mathcal{D}} \rho^{(0)} \overline{\Phi_{N}} \hat{\#} 1\right) .
\end{aligned}
$$

Once these equations are solved the other field components can be calculated from $\gamma_{N}^{H(0)}$ and $\gamma_{N}^{E(0)}$ and their derivatives. Thus (65),(53), (59) and (63) yield

$$
\begin{aligned}
V_{N}^{E(0)} & =\frac{1}{\beta_{N}^{2}}\left(\gamma_{N}^{E(0) \prime}-\frac{1}{\mathcal{N}_{N}^{2} \mu Y^{2}} \int_{\mathcal{D}} \rho^{(0)} \overline{\Phi_{N}} \hat{\#} 1\right) \\
V_{N}^{H(0)} & =\frac{\mu}{\alpha_{N}^{2}} \dot{\gamma}_{N}^{H(0)} \\
I_{N}^{E(0)} & =-\frac{1}{\beta_{N}^{2}}\left(\mu Y^{2} \dot{\gamma}_{N}^{E(0)}+\frac{1}{\mathcal{N}_{N}^{2}} \int_{\mathcal{D}} J_{0}^{(0)} \overline{\Phi_{N}} \hat{\#} 1\right), \\
I_{N}^{H(0)} & =\frac{1}{\alpha_{N}^{2}} \gamma_{N}^{H(0) \prime} .
\end{aligned}
$$

These solutions are consistent for sources satisfying (17). 


\section{Decoupling to First Order}

The first order equations involve the solutions to the lowest order fields. Similar to the case above the equation for $\gamma_{M}^{H(1)}$ follows by substituting (56) and (64) into (60):

$$
\begin{aligned}
\ddot{\gamma}_{M}^{H(1)}-c^{2} \gamma_{M}^{H(1) \prime \prime}+c^{2} \alpha_{M}^{2} \gamma_{M}^{H(1)} \\
=-\frac{c^{2} \kappa_{0}(z)}{\mathcal{M}_{M}^{2}} \sum_{N}^{\prime}\left(I_{N}^{E(0) \prime}-\mu Y^{2} \dot{V}_{N}^{E(0)}\right) G_{M, N}^{\bar{\Psi}, \Phi}-\frac{c^{2}}{\mathcal{M}_{M}^{2}} \kappa_{0}^{\prime}(z) \sum_{N}^{\prime} I_{N}^{E(0)} G_{M, N}^{\bar{\Psi}, \Phi} \\
-\frac{c^{2}}{\mathcal{M}_{M}^{2}} \int_{\mathcal{D}}\left(J_{\theta}^{(1)}-J_{\theta}^{(0)} \kappa_{0}(z) r \cos \theta\right)\left(\hat{\#} \boldsymbol{d} \overline{\Psi_{M}}\right) \wedge \boldsymbol{d} r \\
-\frac{c^{2}}{\mathcal{M}_{M}^{2}} \int_{\mathcal{D}} r\left(J_{r}^{(1)}-J_{r}^{(0)} \kappa_{0}(z) r \cos \theta\right)\left(\hat{\#} \boldsymbol{d} \overline{\Psi_{M}}\right) \wedge \boldsymbol{d} \theta \\
+\frac{c^{2}}{\mathcal{M}_{M}^{2}} \frac{\partial}{\partial z} \sum_{N}^{\prime} \kappa_{0}(z)\left\{\left(\gamma_{N}^{H(0) \prime}+\alpha_{N}^{2} I_{N}^{H(0)}\right) E_{M, N}^{\Psi}-I_{N}^{H(0)}\left(C_{M, N}^{\Psi}-D_{M, N}^{\Psi}\right)\right\} \\
-\frac{\kappa_{0}(z)}{\mu \mathcal{M}_{M}^{2}} \frac{\partial}{\partial t} \sum_{N}^{\prime}\left\{V_{N}^{H(0)}\left(C_{M, N}^{\Psi}-D_{M, N}^{\Psi}\right)\right\},
\end{aligned}
$$

where (53) has been used. The equation for $\gamma_{M}^{E(1)}$ follows by substituting (62) and (66) into (54):

$$
\begin{aligned}
\ddot{\gamma}_{M}^{E(1)}-c^{2} \gamma_{M}^{E(1) \prime \prime}+c^{2} \beta_{M}^{2} \gamma_{M}^{E(1)} & -\frac{c^{2} \kappa_{0}(z)}{\mathcal{N}_{M}^{2}} \sum_{N}^{\prime}\left(V_{N}^{H(0) \prime}+\mu \dot{I}_{N}^{H(0)}\right) G_{M, N}^{\bar{\Phi}, \Psi}-\frac{c^{2} \kappa_{0}^{\prime}(z)}{\mathcal{N}_{M}^{2}} \sum_{N}^{\prime} V_{N}^{H(0)} G_{M, N}^{\bar{\Phi}, \Psi} \\
- & \frac{\kappa_{0}(z)}{\mu Y^{2} \mathcal{N}_{M}^{2}} \frac{\partial}{\partial t} \sum_{N}^{\prime}\left\{\left(\beta_{N}^{2} I_{N}^{E(0)}+\mu Y^{2} \dot{\gamma}_{N}^{E(0)}\right) E_{M, N}^{\Phi}-I_{N}^{E(0)}\left(C_{M, N}^{\Phi}-D_{M, N}^{\Phi}\right)\right\} \\
+ & \frac{c^{2}}{\mathcal{N}_{M}^{2}} \frac{\partial}{\partial z} \sum_{N}^{\prime} \kappa_{0}(z)\left\{\left(\beta_{N}^{2} V_{N}^{E(0)}+\gamma_{N}^{E(0) \prime}\right) E_{M, N}^{\Phi}-V_{N}^{E(0)}\left(C_{M, N}^{\Phi}-D_{M, N}^{\Phi}\right)\right\} \\
- & \frac{c^{2}}{\mu Y^{2} \mathcal{N}_{M}^{2}} \frac{\partial}{\partial z} \int_{\mathcal{D}}\left(\rho^{(1)}-\rho^{(0)} \kappa_{0}(z) r \cos \theta\right) \overline{\Phi_{M}} \hat{\#} 1 \\
- & \frac{\mu c^{2}}{\mathcal{N}_{M}^{2}} \frac{\partial}{\partial t} \int_{\mathcal{D}} J_{0}^{(1)} \overline{\Phi_{M}} \hat{\#} 1 .
\end{aligned}
$$

Expressions for the other field components can be expressed in terms of $\gamma_{M}^{H(1)}$ and $\gamma_{M}^{E(1)}$, their derivatives and lower order fields. From (66), (56), (62) and (64) one has

$$
\begin{aligned}
V_{M}^{E(1)} & =\frac{1}{\beta_{M}^{2}}\left[\gamma_{M}^{E(1) \prime}-\frac{1}{\mu Y^{2} \mathcal{N}_{M}^{2}} \int_{\mathcal{D}}\left(\rho^{(1)}-\rho^{(0)} \kappa_{0}(z) r \cos \theta\right) \overline{\Phi_{M}} \hat{\#} 1\right. \\
& \left.+\frac{\kappa_{0}(z)}{\mathcal{N}_{M}^{2}} \sum_{N}^{\prime}\left\{\left(V_{N}^{E(0)} \beta_{N}^{2}+\gamma_{N}^{E(0) \prime}\right) E_{M, N}^{\Phi}-V_{N}^{E(0)}\left(C_{M, N}^{\Phi}-D_{M, N}^{\Phi}\right)\right\}\right], \\
V_{M}^{H(1)} & =\frac{1}{\alpha_{M}^{2}}\left\{\mu \dot{\gamma}_{M}^{H(1)}+\frac{\kappa_{0}(z)}{\mathcal{M}_{M}^{2}} \sum_{N}^{\prime} V_{N}^{H(0)}\left(C_{M, N}^{\Psi}-D_{M, N}^{\Psi}\right)\right\}, \\
I_{M}^{E(1)} & =-\frac{1}{\beta_{M}^{2}}\left[\mu Y^{2} \dot{\gamma}_{M}^{E(1)}+\frac{\kappa_{0}(z)}{\mathcal{N}_{M}^{2}} \sum_{N}^{\prime}\left\{\left(\beta_{N}^{2} I_{N}^{E(0)}+\mu Y^{2} \dot{\gamma}_{N}^{E(0)}\right) E_{M, N}^{\Phi}\right.\right. \\
- & \left.\left.I_{N}^{E(0)}\left(C_{M, N}^{\Phi}-D_{M, N}^{\Phi}\right)\right\}+\frac{1}{\mathcal{N}_{M}^{2}} \int_{\mathcal{D}} J_{0}^{(1)} \overline{\Phi_{M}} \hat{\#} 1\right] \\
I_{M}^{H(1)} & =\frac{1}{\alpha_{M}^{2}}\left[\gamma_{M}^{H(1) \prime}+\frac{\kappa_{0}(z)}{\mathcal{M}_{M}^{2}} \sum_{N}^{\prime}\left\{\left(\gamma_{N}^{H(0) \prime}+\alpha_{N}^{2} I_{N}^{H(0)}\right) E_{M, N}^{\Psi}\right.\right. \\
- & \left.I_{N}^{H(0)}\left(C_{M, N}^{\Psi}-D_{M, N}^{\Psi}\right)\right\} .
\end{aligned}
$$




\section{General Solutions}

In $₫ \mathrm{VD} 1$ and $\mathrm{VD} 2$ the problem of solving (26), (27), (28), (29) has been reduced to solving an initial-value problem for the decoupled fields $\gamma_{N}^{H(0)}, \gamma_{N}^{H(1)}, \gamma_{N}^{E(0)}$ and $\gamma_{N}^{E(1)}$. For some real constant $\sigma>0$ each satisfies a secondorder hyperbolic partial differential equation in the independent variables $(t, z)$, of the form:

$$
\ddot{f}-c^{2} f^{\prime \prime}+c^{2} \sigma^{2} f=g,
$$

for some prescribed source function $g$. The causal solution of this partial differential equation for $t>0$, with prescribed values of $f(0, z)$ and $\dot{f}(0, z)$, has been exhaustively studied in the literature, see e.g. [12]. If the data and sources are sufficiently smooth the general solution may be expressed in the form

$$
f(t, z)=\mathcal{H}_{\sigma}\left[f^{i n i t}\right](t, z)+\mathcal{I}_{\sigma}[g](t, z),
$$

where

$$
\begin{aligned}
& \mathcal{H}_{\sigma}\left[f^{i n i t}\right](t, z):=\frac{1}{2}\{f(0, z-c t)+f(0, z+c t)\} \\
& +\frac{1}{2 c} \int_{z-c t}^{z+c t} d \zeta \dot{f}(0, \zeta) J_{0}\left(\sigma \sqrt{c^{2} t^{2}-(z-\zeta)^{2}}\right) \\
& -\frac{c t \sigma}{2} \int_{z-c t}^{z+c t} d \zeta f(0, \zeta) \frac{J_{1}\left(\sigma \sqrt{c^{2} t^{2}-(z-\zeta)^{2}}\right)}{\sqrt{c^{2} t^{2}-(z-\zeta)^{2}}}
\end{aligned}
$$

and

$$
\mathcal{I}_{\sigma}[g](t, z):=\frac{1}{2 c} \int_{0}^{t} d t^{\prime} \int_{z-c\left(t-t^{\prime}\right)}^{z+c\left(t-t^{\prime}\right)} d \zeta g\left(t^{\prime}, \zeta\right) J_{0}\left(\sigma \sqrt{c^{2}\left(t-t^{\prime}\right)^{2}-(z-\zeta)^{2}}\right)
$$

The functions $f(0, z), \dot{f}(0, z)$ constitute the initial $t=0$ Cauchy data in this solution and determine the $\mathcal{H}_{\sigma}$ contribution above. Typically, in an accelerating device, lowest order contributions include externally applied piecewise established magnetostatic and RF fields that are together used to guide and accelerate charges along the beam tube. In the following we assume that all $\mathcal{H}_{\sigma}$ contributions to the field solutions arise in lowest order.

\section{E. Electromagnetic Power from Smooth Sources}

In the general situation it is seen that all zero and first order fields can be calculated in terms of finite range integrals involving Bessel functions. It is of some interest to calculate how the instantaneous electromagnetic power flux depends on the first order curvature correction to that in a straight cylinder with smooth sources. This is obtained by integrating the Poynting vector field over the cross-section $\mathcal{D}$ at an arbitrary point with coordinate $z$. In terms of the Poynting 2-form

$$
\underset{(2)}{\mathbf{S}}(\epsilon, t, z, r, \theta):=\underset{(1)}{\mathbf{e}}(\epsilon, t, z, r, \theta) \wedge \underset{(1)}{\mathbf{h}}(\epsilon, t, z, r, \theta),
$$

such instantaneous power $w(\epsilon, t, z)$ is obtained by integrating $\underset{(2)}{\mathbf{S}}$ over $\mathcal{D}$ :

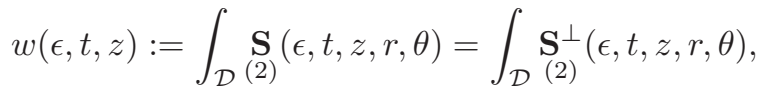

where $\underset{(2)}{\mathbf{S}^{\perp}}$ is the 2 -form that does not contain $\boldsymbol{d} z$ in $\underset{(2)}{\mathbf{S}}$. From the electromagnetic 1-forms, (39) and (40), it follows that

$$
\begin{aligned}
& w(\epsilon, t, z) \\
& =\sum_{N} \sum_{N^{\prime}} V_{N}^{E}(\epsilon, t, z) I_{N^{\prime}}^{E}(\epsilon, t, z) \int_{\mathcal{D}} \boldsymbol{d} \Phi_{N} \wedge \#\left(\boldsymbol{d} z \wedge \boldsymbol{d} \Phi_{N^{\prime}}\right) \\
& \quad+\sum_{M} \sum_{M^{\prime}} V_{M}^{H}(\epsilon, t, z) I_{M^{\prime}}^{H}(\epsilon, t, z) \int_{\mathcal{D}}\left(\#\left(\boldsymbol{d} z \wedge \boldsymbol{d} \Psi_{M}\right)\right) \wedge \boldsymbol{d} \Psi_{M^{\prime}} .
\end{aligned}
$$


Taking into account the symmetries given by (43) and (44), and the fact that the sums over $n^{\prime}, m^{\prime} \in \mathbb{Z}$ are from $-\infty$ to $\infty$, Eq. (80) can be re-written

$$
\begin{aligned}
w= & \sum_{N} \sum_{N^{\prime}} V_{N}^{E}(-1)^{n^{\prime}} \overline{I_{-n^{\prime}, q\left(-n^{\prime}\right)}^{E}} \\
& \times \int_{\mathcal{D}} \boldsymbol{d} \Phi_{N} \wedge \#\left(\boldsymbol{d} z \wedge(-1)^{-n^{\prime}} \boldsymbol{d} \overline{\Phi_{-n^{\prime}, q^{\prime}\left(-n^{\prime}\right)}}\right) \\
& +\sum_{M} \sum_{M^{\prime}} V_{M}^{H}(-1)^{m^{\prime}} \overline{I_{-m^{\prime}, p^{\prime}\left(-m^{\prime}\right)}^{H}} \\
& \times \int_{\mathcal{D}}\left(\#\left(\boldsymbol{d} z \wedge \boldsymbol{d} \Psi_{M}\right)\right) \wedge(-1)^{m^{\prime}} \boldsymbol{d} \overline{\Psi_{-m^{\prime}, p^{\prime}\left(-m^{\prime}\right)}} \\
= & \sum_{N} \sum_{N^{\prime}} V_{N}^{E} \overline{I_{N^{\prime}}^{E}} \int_{\mathcal{D}} \boldsymbol{d} \Phi_{N} \wedge \#\left(\boldsymbol{d} z \wedge \boldsymbol{d} \overline{\Phi_{N^{\prime}}}\right) \\
& +\sum_{M} \sum_{M^{\prime}} V_{M}^{H} \overline{I_{M^{\prime}}^{H}} \int_{\mathcal{D}}\left(\#\left(\boldsymbol{d} z \wedge \boldsymbol{d} \Psi_{M}\right)\right) \wedge \boldsymbol{d} \overline{\Psi_{M^{\prime}}} .
\end{aligned}
$$

Writing

$$
w(\epsilon, t, z)=w^{(0)}(t, z)+\epsilon w^{(1)}(t, z)+\mathcal{O}\left(\epsilon^{2}\right)
$$

one has

$$
\begin{gathered}
w^{(0)}(t, z)=\sum_{N} \sum_{N^{\prime}} V_{N}^{E(0)}(t, z) I_{N^{\prime}}^{E(0)}(t, z) \int_{\mathcal{D}} \boldsymbol{d} \Phi_{N} \wedge \hat{\#} \boldsymbol{d} \Phi_{N^{\prime}} \\
+\sum_{M} \sum_{M^{\prime}} V_{M}^{H(0)}(t, z) I_{M^{\prime}}^{H(0)}(t, z) \int_{\mathcal{D}}\left(\hat{\#} \boldsymbol{d} \Psi_{M}\right) \wedge \boldsymbol{d} \Psi_{M^{\prime}}
\end{gathered}
$$

and

$$
\begin{aligned}
& w^{(1)}(t, z)=\sum_{N} \sum_{N^{\prime}}\left\{V_{N}^{E(0)}(t, z) I_{N^{\prime}}^{E(0)}(t, z) \kappa_{0}(z)\right. \\
& \times \int_{\mathcal{D}} r \cos \theta \boldsymbol{d} \Phi_{N} \wedge \hat{\#} \boldsymbol{d} \Phi_{N^{\prime}} \\
& \left.+\left(V_{N}^{E(0)}(t, z) I_{N^{\prime}}^{E(1)}(t, z)+V_{N}^{E(1)}(t, z) I_{N^{\prime}}^{E(0)}(t, z)\right) \int_{\mathcal{D}} \boldsymbol{d} \Phi_{N} \wedge \hat{\#} \boldsymbol{d} \Phi_{N^{\prime}}\right\} \\
& +\sum_{M} \sum_{M^{\prime}}\left\{V_{M}^{H(0)}(t, z) I_{M^{\prime}}^{H(0)}(t, z) \kappa_{0}(z) \int_{\mathcal{D}} r \cos \theta\left(\hat{\#} \boldsymbol{d} \Psi_{M}\right) \wedge \boldsymbol{d} \Psi_{M^{\prime}}\right. \\
& +\left(V_{M}^{H(0)}(t, z) I_{M^{\prime}}^{H(1)}(t, z)+V_{M}^{H(1)}(t, z) I_{M^{\prime}}^{H(0)}(t, z)\right) \\
& \left.\times \int_{\mathcal{D}}\left(\hat{\#} \boldsymbol{d} \Psi_{M}\right) \wedge \boldsymbol{d} \Psi_{M^{\prime}}\right\} .
\end{aligned}
$$

With the aid of the symmetries, (43) and (44), and the fact that

$$
\int_{\mathcal{D}} \boldsymbol{d} \Phi_{N} \wedge \hat{\#} \boldsymbol{d} \Phi_{N^{\prime}} \propto \delta_{n^{\prime},-n}, \quad \int_{\mathcal{D}}\left(\hat{\#} \boldsymbol{d} \Psi_{M}\right) \wedge \boldsymbol{d} \Psi_{M^{\prime}} \propto \delta_{m^{\prime},-m},
$$

one obtains finally

$$
w^{(0)}=\Re\left\{\sum_{N} \beta_{N}^{2} \mathcal{N}_{N}^{2} V_{N}^{E(0)} \overline{I_{N}^{E(0)}}-\sum_{M} \alpha_{M}^{2} \mathcal{M}_{M}^{2} V_{M}^{H(0)} \overline{I_{M}^{H(0)}}\right\},
$$

where $\Re$ represents the real part of its argument. 
Similarly the first order correction to the power is

$$
\begin{aligned}
w^{(1)}= & \Re\left\{\sum_{N} \beta_{N}^{2} \mathcal{N}_{N}^{2}\left(V_{N}^{E(0)} \overline{I_{N}^{E(1)}}+V_{N}^{E(1)} \overline{I_{N}^{E(0)}}\right)\right. \\
& -\sum_{M} \alpha_{M}^{2} \mathcal{M}_{M}^{2}\left(V_{M}^{H(0)} \overline{I_{M}^{H(1)}}+V_{M}^{H(1)} \overline{I_{M}^{H(0)}}\right) \\
& +\kappa_{0}(z) \sum_{N} \sum_{N^{\prime}} F_{N, N^{\prime}}^{\Phi} V_{N}^{E(0)} \overline{I_{N^{\prime}}^{E(0)}} \\
& \left.-\kappa_{0}(z) \sum_{M} \sum_{M^{\prime}} F_{M, M^{\prime}}^{\Psi} V_{M}^{H(0)} \overline{I_{M^{\prime}}^{H(0)}}\right\} .
\end{aligned}
$$

The analysis above is general and accommodates arbitrary smooth continuous conserved source currents. It should be stressed that in general one must specify how the prescribed sources should depend on both space and time variables so that their perturbative expansions in $\kappa$ can be determined. In the following sections we consider particular sources of relevance to the issues mentioned in the introduction.

\section{SMOOTH LONGITUDINAL CONVECTIVE CURRENTS}

In this section a particular current source is considered. It is generated by an arbitrary smooth convective charge density $\rho$ that is taken independent of $\kappa$. It has components $J_{r}=J_{\theta}=0, J_{0}(z-v t, r, \theta)=v \rho(z-v t, r, \theta)$ with $v$ less than the speed of light. In this source model $J_{0}^{(0)}=J_{0}$ and all higher orders are taken zero. This is a contrived current source but serves as a comparison with the localised sources that will be used to model accelerated bunches of charge in subsequent sections.

\section{A. Lowest Order System with Smooth Longitudinal Currents}

The equations for $\gamma_{N}^{H(0)}$ and $\gamma_{N}^{E(0)}$ follow for these sources from above:

$$
\begin{gathered}
\ddot{\gamma}_{N}^{H(0)}-c^{2} \gamma_{N}^{H(0) \prime \prime}+c^{2} \alpha_{N}^{2} \gamma_{N}^{H(0)}=0 . \\
\ddot{\gamma}_{N}^{E(0)}-c^{2} \gamma_{N}^{E(0) \prime \prime}+c^{2} \beta_{N}^{2} \gamma_{N}^{E(0)}=-\frac{c^{2} \mu}{\mathcal{N}_{N}^{2}}\left(c^{2}-v^{2}\right) \overline{\rho_{N}^{(0) \prime}} .
\end{gathered}
$$

where the conservation relation

$$
\frac{\partial}{\partial t} \rho^{(0)}(z-v t)=-v \frac{\partial}{\partial z} \rho^{(0)}(z-v t),
$$

has been used and for $k \in \mathbb{Z}_{\geq 0}$ we define

$$
\rho_{N}^{(k)}:=\int_{\mathcal{D}} \rho^{(k)} \Phi_{N} \hat{\#} 1 .
$$

In terms of $\gamma_{N}^{H(0)}$ and $\gamma_{N}^{E(0)}$ and the projected convective longitudinal sources the equations (65), (53), (59) and (63) yield

$$
\begin{aligned}
V_{N}^{E(0)} & =\frac{1}{\beta_{N}^{2}}\left(\gamma_{N}^{E(0) \prime}-\frac{1}{\mathcal{N}_{N}^{2} \mu Y^{2}} \rho_{N}^{(0)}\right), \\
V_{N}^{H(0)} & =\frac{\mu}{\alpha_{N}^{2}} \dot{\gamma}_{N}^{H(0)}, \\
I_{N}^{E(0)} & =-\frac{1}{\beta_{N}^{2}}\left(\mu Y^{2} \dot{\gamma}_{N}^{E(0)}+\frac{v}{\mathcal{N}_{N}^{2}} \overline{\rho_{N}^{(0)}}\right), \\
I_{N}^{H(0)} & =\frac{1}{\alpha_{N}^{2}} \gamma_{N}^{H(0) \prime},
\end{aligned}
$$


and from (83), (88), (89), (90), and (91) the lowest order contribution to the power flux becomes

$$
\begin{aligned}
w^{(0)}(t, z)= & \Re\left\{\sum _ { N } \left(\frac{v\left|\rho_{N}^{(0)}\right|^{2}}{\mathcal{N}_{N}^{2} \beta_{N}^{2} \mu Y^{2}}+\frac{\overline{\dot{\gamma}_{N}^{E(0)}}}{\beta_{N}^{2}} \rho_{N}^{(0)}-\frac{v \gamma_{N}^{E(0) \prime}}{\beta_{N}^{2}} \rho_{N}^{(0)}\right.\right. \\
& \left.\left.-\frac{\mu Y^{2}}{\beta_{N}^{2}} \mathcal{N}_{N}^{2} \gamma_{N}^{E(0) \prime} \overline{\dot{\gamma}_{N}^{E(0)}}\right)-\mu \sum_{M} \frac{\mathcal{M}_{M}^{2}}{\alpha_{M}^{2}} \dot{\gamma}_{M}^{H(0)} \overline{\gamma_{M}^{H(0) \prime}}\right\} .
\end{aligned}
$$

\section{B. First Order System with Smooth Longitudinal Currents}

The equations for $\gamma_{M}^{H(1)}$ and $\gamma_{M}^{E(1)}$ follow similarly. From (56), (64), 60) one has

$$
\begin{aligned}
& \ddot{\gamma}_{M}^{H(1)}-c^{2} \gamma_{M}^{H(1) \prime \prime}+c^{2} \alpha_{M}^{2} \gamma_{M}^{H(1)} \\
& =-\frac{c^{2} \kappa_{0}(z)}{\mathcal{M}_{M}^{2}} \sum_{N}^{\prime}\left(I_{N}^{E(0) \prime}-\mu Y^{2} \dot{V}_{N}^{E(0)}\right) G_{M, N}^{\bar{\Psi}, \Phi}-\frac{c^{2} \kappa_{0}^{\prime}(z)}{\mathcal{M}_{M}^{2}} \sum_{N}^{\prime} I_{N}^{E(0)} G_{M, N}^{\bar{\Psi}, \Phi} \\
& -\frac{c^{2} \kappa_{0}(z)}{\mathcal{M}_{M}^{2}} \sum_{N}^{\prime}\left\{\left(\mu Y^{2} \dot{V}_{N}^{H(0)}+I_{N}^{H(0) \prime}\right)\left(C_{M, N}^{\Psi}-D_{M, N}^{\Psi}\right)\right. \\
& \left.\quad-\left(\gamma_{N}^{H(0) \prime \prime}+\alpha_{N}^{2} I_{N}^{H(0) \prime}\right) E_{M, N}^{\Psi}\right\}+\frac{c^{2} \kappa_{0}^{\prime}(z)}{\mathcal{M}_{M}^{2}} \sum_{N}^{\prime}\left\{\left(\gamma_{N}^{H(0) \prime}\right.\right. \\
& \left.\left.\quad+\alpha_{N}^{2} I_{N}^{H(0)}\right) E_{M, N}^{\Psi}-I_{N}^{H(0)}\left(C_{M, N}^{\Psi}-D_{M, N}^{\Psi}\right)\right\} .
\end{aligned}
$$

and (62), (66), yield

$$
\begin{aligned}
& \ddot{\gamma}_{M}^{E(1)}-c^{2} \gamma_{M}^{E(1) \prime \prime}+c^{2} \beta_{M}^{2} \gamma_{M}^{E(1)} \\
& =-\frac{c^{2} \kappa_{0}(z)}{\mathcal{N}_{M}^{2}} \sum_{N}^{\prime}\left(V_{N}^{H(0) \prime}+\mu \dot{I}_{N}^{H(0)}\right) G_{M, N}^{\bar{\Phi}, \Psi}-\frac{c^{2} \kappa_{0}^{\prime}(z)}{\mathcal{N}_{M}^{2}} \sum_{N}^{\prime} V_{N}^{H(0)} G_{M, N}^{\bar{\Phi}, \Psi} \\
& -\frac{c^{2} \mu \kappa_{0}(z)}{\mathcal{N}_{M}^{2}} \sum_{N}^{\prime}\left\{\left(\beta_{N}^{2} \dot{I}_{N}^{E(0)}+\mu Y^{2} \ddot{\gamma}_{N}^{E(0)}\right)-\dot{I}_{N}^{E(0)}\left(C_{M, N}^{\Phi}-D_{M, N}^{\Phi}\right)\right\} \\
& +\frac{c^{2} \kappa_{0}(z)}{\mathcal{N}_{M}^{2}} \sum_{N}^{\prime}\left\{\left(\beta_{N}^{2} V_{N}^{E(0) \prime}+\gamma_{N}^{E(0) \prime \prime}\right) E_{M, N}^{\Phi}-V_{N}^{E(0) \prime}\left(C_{M, N}^{\Phi}-D_{M, N}^{\Phi}\right)\right\} \\
& +\frac{\mu c^{4}}{\mathcal{N}_{M}^{2}} \frac{\partial}{\partial z} \int_{\mathcal{D}}\left(\rho^{(0)} \kappa_{0}(z) r \cos \theta\right) \bar{\Phi} \hat{\Phi}_{M} \# 1+\frac{c^{2} \kappa_{0}^{\prime}(z)}{\mathcal{N}_{M}^{2}} \sum_{N}^{\prime}\left\{\left(\beta_{N}^{2} V_{N}^{E(0)}\right.\right. \\
& \left.\left.\quad+\gamma_{N}^{E(0) \prime}\right) E_{M, N}^{\Phi}-V_{N}^{E(0)}\left(C_{M, N}^{\Phi}-D_{M, N}^{\Phi}\right)\right\} .
\end{aligned}
$$

The right hand sides of these expressions for $\gamma_{M}^{H(1)}$ and $\gamma_{M}^{E(1)}$ can be rewritten in terms of the lower order fields $\gamma_{N}^{E(0)}, \gamma_{N}^{H(0)}$ and the sources. Thus the following terms in (93) can be expressed as

$$
\begin{aligned}
& I_{N}^{E(0) \prime}-\mu Y^{2} \dot{V}_{N}^{E(0)}=-2\left(\frac{\mu Y^{2}}{\beta_{N}^{2}} \dot{\gamma}_{N}^{E(0) \prime}+\frac{v}{\mathcal{N}_{N}^{2} \beta_{N}^{2}} \overline{\rho_{N}^{(0) \prime}}\right) \\
& \mu Y^{2} \dot{V}_{N}^{H(0)}+I_{N}^{H(0) \prime}=\frac{1}{\alpha_{N}^{2}}\left(\frac{1}{c^{2}} \ddot{\gamma}_{N}^{H(0)}+\gamma_{N}^{H(0) \prime \prime}\right) \\
& \gamma_{N}^{H(0) \prime}+\alpha_{N}^{2} I_{N}^{H(0)}=2 \gamma_{N}^{H(0) \prime} .
\end{aligned}
$$


Similarly the following terms in (94) can be expressed as

$$
\begin{aligned}
& V_{N}^{H(0) \prime}+\mu \dot{I}_{N}^{H(0)}=2 \frac{\mu}{\alpha_{N}^{2}} \dot{\gamma}_{N}^{H(0) \prime} \\
& \beta_{N}^{2} \dot{I}_{N}^{E(0)}+\mu Y^{2} \ddot{\gamma}_{N}^{E(0)}=\frac{v^{2}}{\mathcal{N}_{N}^{2}} \overline{\rho_{N}^{(0) \prime}}, \\
& \dot{I}_{N}^{E(0)}=-\frac{1}{\beta_{N}^{2}}\left(\mu Y^{2} \ddot{\gamma}_{N}^{E(0)}-\frac{v^{2}}{\mathcal{N}_{N}^{2}} \overline{\rho_{N}^{(0) \prime}}\right), \\
& \beta_{N}^{2} V_{N}^{E(0)}+\gamma_{N}^{E(0) \prime}=2 \gamma_{N}^{E(0) \prime}-\frac{1}{\mathcal{N}_{N}^{2} \mu Y^{2}} \overline{\rho_{N}^{(0)}}, \\
& V_{N}^{E(0)}=\frac{1}{\beta_{N}^{2}}\left(\gamma_{N}^{E(0) \prime}-\frac{1}{\mathcal{N}_{N}^{2} \mu Y^{2}} \overline{\rho_{N}^{(0)}}\right) .
\end{aligned}
$$

Finally once $\gamma_{M}^{H(1)}$ and $\gamma_{M}^{E(1)}$ have been determined from these decoupled equations it follows from (66), (56), (62), (64) that the remaining fields can be readily determined as:

$$
\begin{aligned}
V_{M}^{E(1)}= & \frac{1}{\beta_{M}^{2}}\left[\gamma_{M}^{E(1) \prime}+\frac{1}{\mu Y^{2} \mathcal{N}_{M}^{2}} \int_{\mathcal{D}}\left(\rho^{(0)} \kappa_{0}(z) r \cos \theta\right) \overline{\Phi_{M}} \hat{\#} 1\right. \\
& \left.+\frac{\kappa_{0}(z)}{\mathcal{N}_{M}^{2}} \sum_{N}^{\prime}\left\{\left(V_{N}^{E(0)} \beta_{N}^{2}+\gamma_{N}^{E(0) \prime}\right) E_{M, N}^{\Phi}-V_{N}^{E(0)}\left(C_{M, N}^{\Phi}-D_{M, N}^{\Phi}\right)\right\}\right] \\
V_{M}^{H(1)}= & \frac{1}{\alpha_{M}^{2}}\left\{\mu \dot{\gamma}_{M}^{H(1)}+\frac{\kappa_{0}(z)}{\mathcal{M}_{M}^{2}} \sum_{N}^{\prime} V_{N}^{H(0)}\left(C_{M, N}^{\Psi}-D_{M, N}^{\Psi}\right)\right\} \\
I_{M}^{E(1)}= & -\frac{1}{\beta_{M}^{2}}\left[\mu Y^{2} \dot{\gamma}_{M}^{E(1)}+\frac{\kappa_{0}(z)}{\mathcal{N}_{M}^{2}} \sum_{N}^{\prime}\left\{\left(\beta_{N}^{2} I_{N}^{E(0)}+\mu Y^{2} \dot{\gamma}_{N}^{E(0)}\right) E_{M, N}^{\Phi}\right.\right. \\
& \left.\left.-I_{N}^{E(0)}\left(C_{M, N}^{\Phi}-D_{M, N}^{\Phi}\right)\right\}\right] \\
I_{M}^{H(1)}= & \frac{1}{\alpha_{M}^{2}}\left[\gamma_{M}^{H(1) \prime}+\frac{\kappa_{0}(z)}{\mathcal{M}_{M}^{2}} \sum_{N}^{\prime}\left\{\left(\gamma_{N}^{H(0) \prime}+\alpha_{N}^{2} I_{N}^{H(0)}\right) E_{M, N}^{\Psi}\right.\right. \\
& \left.-I_{N}^{H(0)}\left(C_{M, N}^{\Psi}-D_{M, N}^{\Psi}\right)\right\} .
\end{aligned}
$$

\section{Radiated Power Dependence on Local Curvature}

A straightforward but tedious calculation leads to the dependence on local curvature of the radiated power (84) for the convective source model above, in terms of $\gamma_{N}^{H(0)}, \gamma_{N}^{E(0)}, \gamma_{N}^{H(1)}, \gamma_{N}^{E(1)}$, various overlap coefficients and projections of $\rho$.

Each term in (84) can be reduced as follows:

$$
\begin{aligned}
& \sum_{N} \beta_{N}^{2} \mathcal{N}_{N}^{2} V_{N}^{E(0)} \overline{I_{N}^{E(1)}}=-\sum_{N} \frac{\mathcal{N}_{N}^{2}}{\beta_{N}^{2}}\left(\gamma_{N}^{E(0) \prime}-\frac{1}{\mathcal{N}_{N}^{2} \mu Y^{2}} \overline{\rho_{N}^{(0)}}\right) \\
& \times\left[\mu Y^{2} \overline{\dot{\gamma}_{N}^{E(1)}}+\frac{\kappa_{0}(z)}{\mathcal{N}_{N}^{2}} \sum_{M}^{\prime}\left\{-\frac{E_{M, N}^{\Phi}}{\mathcal{N}_{N}^{2}} \rho_{M}^{(0)}\right.\right. \\
& \left.\left.\quad+\frac{C_{N, M}^{\Phi}-D_{N, M}^{\Phi}}{\beta_{M}^{2}}\left(\mu Y^{2} \overline{\dot{\gamma}_{M}^{E(0)}}+\frac{v}{\mathcal{N}_{M}^{2}} \rho_{M}^{(0)}\right)\right\}\right]
\end{aligned}
$$




$$
\begin{aligned}
& \sum_{N} \beta_{N}^{2} \mathcal{N}_{N}^{2} V_{N}^{E(1)} \overline{I_{N}^{E(0)}} \\
& =-\sum_{N} \frac{\mathcal{N}_{N}^{2}}{\beta_{N}^{2}}\left[\gamma_{N}^{E(1) \prime}+\frac{1}{\mu Y^{2} \mathcal{N}_{N}^{2}} \int_{\mathcal{D}} \rho^{(0)} \kappa_{0}(z) r \cos \theta \overline{\Phi_{N}} \hat{\#} 1\right. \\
& +\frac{\kappa_{0}(z)}{\mathcal{N}_{N}^{2}} \sum_{M}^{\prime}\left\{\left(2 E_{N, M}^{\Phi}-\frac{C_{M, N}^{\Phi}-D_{M, N}^{\Phi}}{\beta_{M}^{2}}\right) \gamma_{M}^{E(0) \prime}\right. \\
& \left.\left.+\left(-E_{N, M}^{\Phi}+\frac{C_{N, M}^{\Phi}-D_{N, M}^{\Phi}}{\beta_{M}^{2}}\right) \overline{\frac{\rho_{M}^{(0)}}{\mathcal{N}_{M}^{2}}}\right\}\right]\left(\overline{\dot{\gamma}_{N}^{E(0)}}+\frac{v \rho_{N}^{(0)}}{\mathcal{N}_{N}^{2} \mu Y^{2}}\right), \\
& \sum_{M} \alpha_{M}^{2} \mathcal{M}_{M}^{2} V_{M}^{H(0)} \overline{I_{M}^{H(1)}}=\mu \sum_{M} \frac{\mathcal{M}_{M}^{2}}{\alpha_{M}^{2}} \dot{\gamma}_{M}^{H(0)}\left\{\overline{\gamma_{M}^{H(1) \prime}}\right. \\
& \left.+\frac{\kappa_{0}(z)}{\mathcal{M}_{M}^{2}} \sum_{N}^{\prime}\left(2 E_{M, N}^{\Psi}-\frac{C_{M, N}^{\Psi}-D_{M, N}^{\Psi}}{\alpha_{N}^{2}}\right) \overline{\gamma_{N}^{H(0) \prime}}\right\}, \\
& \sum_{M} \alpha_{M}^{2} \mathcal{M}_{M}^{2} V_{M}^{H(1)} \overline{I_{M}^{H(0)}} \\
& =\mu \sum_{M} \frac{\mathcal{M}_{M}^{2}}{\alpha_{M}^{2}}\left(\dot{\gamma}_{M}^{H(1)}+\frac{\kappa_{0}(z)}{\mathcal{M}_{M}^{2}} \sum_{N}^{\prime} \frac{C_{M, N}^{\Psi}-D_{M, N}^{\Psi}}{\alpha_{N}^{2}} \dot{\gamma}_{N}^{H(0)}\right) \overline{\gamma_{M}^{H(0) \prime}}, \\
& \sum_{N} \sum_{N^{\prime}} F_{N, N^{\prime}}^{\Phi} V_{N}^{E(0)} \overline{I_{N^{\prime}}^{E(0)}} \\
& =-\sum_{N} \sum_{N^{\prime}} \frac{F_{N, N^{\prime}}^{\Phi}}{\beta_{N}^{2} \beta_{N^{\prime}}^{2}}\left(\gamma_{N}^{E(0) \prime}-\frac{\overline{\rho_{N}^{(0)}}}{\mathcal{N}_{N}^{2} \mu Y^{2}}\right)\left(\mu Y^{2} \overline{\dot{\gamma}_{N^{\prime}}^{E(0)}}+\frac{v \rho_{N^{\prime}}^{(0)}}{\mathcal{N}_{N^{\prime}}^{2}}\right) .
\end{aligned}
$$

Finally,

$$
\sum_{M} \sum_{M^{\prime}} F_{M, M^{\prime}}^{\Psi} V_{M}^{H(0)} \overline{I_{M^{\prime}}^{H(0)}}=\mu \sum_{M} \sum_{M^{\prime}} F_{M, M^{\prime}}^{\Psi} \frac{\dot{\gamma}_{M}^{H(0)} \overline{\gamma_{M^{\prime}}^{H(0) \prime}}}{\alpha_{M}^{2} \alpha_{M^{\prime}}^{2}} .
$$

\section{MOVING POINT CHARGE SOURCE}

In this section the above formalism is applied to the determination of the fields produced by the motion of a single point charge source moving in a curved beam pipe. A full account would involve generalising the formalism to accommodate fields from point distributional sources. Following established custom the formalism is extended here by modelling a point source as a moving Dirac distribution on $\mathcal{U}$. One can then explicitly remove some of the integrations that arise in the smooth continuous charge source model. However a point charge implies singularities in

the electromagnetic fields and these should not be ignored. The distributional charge density must model a charged particle with constant electric charge $Q_{t o t}$ so we demand that

$$
\int_{\mathcal{U}} \rho \# 1=Q_{t o t}
$$

Furthermore the motion of the charge is maintained (e.g. by externally applied magnetostatic fields) on a curved path parallel to the design-orbit with curvature $\kappa(z)$ and constant speed $v$. These conditions are satisfied if

$$
\rho\left(\epsilon, t, z, x_{1}, x_{2}\right)=Q(\epsilon, t) \delta\left(x_{1}-x_{1,0}\right) \delta\left(x_{2}-x_{2,0}\right) \delta(z-v t),
$$

with

$$
Q(\epsilon, t):=\frac{Q_{t o t}}{1-\epsilon \kappa_{0}(v t) x_{1,0}}=Q_{t o t}+\epsilon \kappa_{0}(v t) x_{1,0} Q_{t o t}+\mathcal{O}\left(\epsilon^{2}\right)
$$


in terms of the symbolic representation of the Cartesian three-dimensional Dirac distribution with moving point support at

$\left(x_{1,0}, x_{2,0}, v t\right)=\left(r_{0} \cos \theta_{0}, r_{0} \sin \theta_{0}, v t\right)$, determining the location of the point charge in $\mathcal{U}$ at time $t$.

Thus it follows from (102) that

$$
\begin{aligned}
\rho & =\rho^{(0)}+\epsilon \rho^{(1)}+\mathcal{O}\left(\epsilon^{2}\right) \\
\rho^{(0)}\left(z-v t, x_{1}, x_{2}\right) & =Q_{t o t} \delta\left(x_{1}-x_{1,0}\right) \delta\left(x_{2}-x_{2,0}\right) \delta(z-v t), \\
\rho^{(1)}\left(t, z, x_{1}, x_{2}\right) & =\kappa_{0}(v t) x_{1,0} Q_{t o t} \delta\left(x_{1}-x_{1,0}\right) \delta\left(x_{2}-x_{2,0}\right) \delta(z-v t) .
\end{aligned}
$$

In adapted coordinates

$$
(\rho \hat{\#} 1)(\epsilon, t, z, r, \theta)=Q(\epsilon, t) \frac{\delta\left(r-r_{0}\right)}{r} \delta\left(\theta-\theta_{0}\right) \delta(z-v t) r \boldsymbol{d} r \wedge \boldsymbol{d} \theta
$$

The associated electric current components are $J_{r}=J_{\theta}=0$ and

$$
\begin{aligned}
J_{0}^{(0)}=v \rho^{(0)}= & v Q_{t o t} \delta\left(x_{1}-x_{1,0}\right) \delta\left(x_{2}-x_{2,0}\right) \delta(z-v t), \\
J_{0}^{(1)}=v \rho^{(1)}= & v \kappa_{0}(v t) x_{1,0} Q_{t o t} \\
& \times \delta\left(x_{1}-x_{1,0}\right) \delta\left(x_{2}-x_{2,0}\right) \delta(z-v t) .
\end{aligned}
$$

Note that in this source model both $J_{0}^{(0)}, J_{0}^{(1)}$ and $\rho^{(0)}, \rho^{(1)}$ are non-zero with all higher orders zero.

\section{A. Lowest Order Fields from a Moving Point Charge}

Using this distributional model the projected sources in (86) can be evaluated as

$$
\int_{\mathcal{D}} \frac{\partial}{\partial z} \rho^{(0)}(z-v t, r, \theta) \overline{\Phi_{N}} \hat{\#} 1=Q_{t o t} J_{n}\left(x_{q(n)} \frac{r_{0}}{a}\right) e^{-i n \theta_{0}} \delta^{\prime}(z-v t) .
$$

so the solutions for $\gamma_{N}^{H(0)}$ and $\gamma_{N}^{E(0)}$ following from (176) are

$$
\begin{aligned}
\gamma_{N}^{H(0)}(t, z) & =\mathcal{H}_{\alpha_{N}}\left[\gamma_{N}^{H(0) \text { init }}\right](t, z), \\
\gamma_{N}^{E(0)}(t, z) & =\mathcal{H}_{\beta_{N}}\left[\gamma_{N}^{E(0) i n i t}\right](t, z) \\
- & \frac{c^{2} \mu}{\mathcal{N}_{N}^{2}}\left(c^{2}-v^{2}\right) Q_{t o t} J_{n}\left(x_{q(n)} \frac{r_{0}}{a}\right) e^{-i n \theta_{0}} \Upsilon_{N}(t, z),
\end{aligned}
$$

where

$$
\Upsilon_{N}(t, z):=\int_{0}^{t} d t^{\prime} \int_{z-c\left(t-t^{\prime}\right)}^{z+c\left(t-t^{\prime}\right)} d \zeta \delta^{\prime}\left(\zeta-v t^{\prime}\right) J_{0}\left(\beta_{N} \sqrt{c^{2}\left(t-t^{\prime}\right)^{2}-(z-\zeta)^{2}}\right)
$$

and $\mathcal{H}_{\alpha_{N}}$ is given by (77). For $v>0$ (See Fig 1 it is shown in Appendix B that:

- for $z \leq-c t$, (Outside the Regions $R_{1}$ and $R_{2}$ )

$$
\Upsilon_{N}(t, z)=0
$$

- for $-c t<z \leq v t,\left(\right.$ Region $\left.R_{2}\right)$

$$
\Upsilon_{N}(t, z)=\beta_{N} \int_{0}^{t_{+}^{\prime}(t, z)} d t^{\prime} \frac{\left(z-v t^{\prime}\right) J_{1}\left(\beta_{N} s\left(t, z \mid t^{\prime}\right)\right)}{s\left(t, z \mid t^{\prime}\right)},
$$

- for $v t \leq z<c t,\left(\right.$ Region $\left.R_{1}\right)$

$$
\Upsilon_{N}(t, z)=\beta_{N} \int_{0}^{t_{-}^{\prime}(t, z)} d t^{\prime} \frac{\left(z-v t^{\prime}\right) J_{1}\left(\beta_{N} s\left(t, z \mid t^{\prime}\right)\right)}{s\left(t, z \mid t^{\prime}\right)},
$$


- for $z \geq c t$, (Outside the regions $R_{1}$ and $R_{2}$ )

$$
\Upsilon_{N}(t, z)=0
$$

where

$$
\begin{aligned}
& t_{+}^{\prime}(t, z):=\frac{t+z / c}{1+v / c}, \quad t_{-}^{\prime}(t, z):=\frac{t-z / c}{1-v / c}, \\
& \text { and } \quad s\left(t, z \mid t^{\prime}\right):=\sqrt{c^{2}\left(t-t^{\prime}\right)^{2}-\left(z-v t^{\prime}\right)^{2}} .
\end{aligned}
$$

The field components $V_{N}^{E(0)}, V_{N}^{H(0)}, I_{N}^{E(0)}, I_{N}^{H(0)}$ follow from (69), (70), (171), (72) in terms of the solutions for $\gamma_{N}^{H(0)}, \gamma_{N}^{E(0)}$.

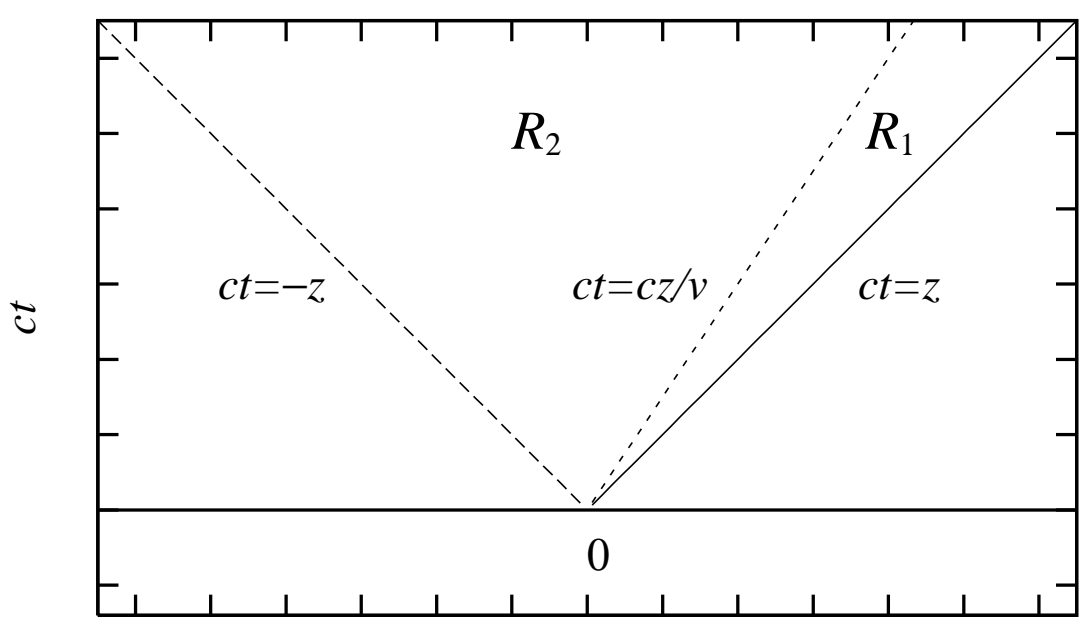

$z$

FIG. 1: Domains for $\Upsilon_{N}(t, z)$ and $A_{\sigma}(t, z), B_{\sigma}(t, z)$ in $\$ \mathrm{VIIB} R_{1}$ is the triangle in the upper right-hand corner $(v t \leq z<c t)$. $R_{2}$ is the adjacent large triangle $(-c t<z \leq v t)$ where the source world-line has $z=v t$.

\section{Lowest Order Contribution to the Instantaneous Power from a Moving Point Charge}

We note from (92) that the lowest order contribution $w^{(0)}$ to the instantaneous power contains terms derived from the projections

$$
\int_{\mathcal{D}} \rho^{(0)}(z-v t, r, \theta) \overline{\Phi_{N}} \hat{\#} 1=Q_{t o t} J_{n}\left(x_{q(n)} \frac{r_{0}}{a}\right) e^{-i n \theta_{0}} \delta(z-v t) .
$$

Such distributional contributions are absent in pipe cross-sections that do not contain the point source. Thus for sections with $z \neq v t$ the instantaneous power is given to lowest order as

$$
\breve{w}^{(0)}=-\Re\left\{\mu Y^{2} \sum_{N} \frac{\mathcal{N}_{N}^{2}}{\beta_{N}^{2}} \gamma_{N}^{E(0) \prime} \overline{\dot{\gamma}_{N}^{E(0)}}+\mu \sum_{M} \frac{\mathcal{M}_{M}^{2}}{\alpha_{M}^{2}} \dot{\gamma}_{M}^{H(0)} \overline{\gamma_{M}^{H(0) \prime}}\right\},
$$

explicitly in terms of the above solutions. This is independent of the curvature $\kappa$.

\section{B. First Order Fields from a Moving Point Charge}

The explicit computation of the contribution of the radiated power to the next order, involving $\kappa$, is somewhat more complicated since it requires the solutions to the zeroth-order fields as sources for the first-order fields as well 
as evaluation of projected sources that now involve $\rho^{(1)}$. The equations for $\gamma_{M}^{H(1)}$ and $\gamma_{M}^{E(1)}$ also contain additional source terms from these first order contributions to the sources. From (56), (64), (60) one has

$$
\begin{aligned}
& \ddot{\gamma}_{M}^{H(1)}-c^{2} \gamma_{M}^{H(1) \prime \prime}+c^{2} \alpha_{M}^{2} \gamma_{M}^{H(1)} \\
& =-\frac{c^{2} \kappa_{0}(z)}{\mathcal{M}_{M}^{2}} \sum_{N}^{\prime}\left(I_{N}^{E(0) \prime}-\mu Y^{2} \dot{V}_{N}^{E(0)}\right) G_{M, N}^{\bar{\Psi}, \Phi}-\frac{c^{2} \kappa_{0}^{\prime}(z)}{\mathcal{M}_{M}^{2}} \sum_{N}^{\prime} I_{N}^{E(0)} G_{M, N}^{\bar{\Psi}, \Phi} \\
& -\frac{c^{2} \kappa_{0}(z)}{\mathcal{M}_{M}^{2}} \sum_{N}^{\prime}\left\{\left(\mu Y^{2} \dot{V}_{N}^{H(0)}+I_{N}^{H(0) \prime}\right)\left(C_{M, N}^{\Psi}-D_{M, N}^{\Psi}\right)\right. \\
& \left.\quad-\left(\gamma_{N}^{H(0) \prime \prime}+\alpha_{N}^{2} I_{N}^{H(0) \prime}\right) E_{M, N}^{\Psi}\right\}+\frac{c^{2} \kappa_{0}^{\prime}(z)}{\mathcal{M}_{M}^{2}} \sum_{N}^{\prime}\left\{\left(\gamma_{N}^{H(0) \prime}\right.\right. \\
& \left.\left.\quad+\alpha_{N}^{2} I_{N}^{H(0)}\right) E_{M, N}^{\Psi}-I_{N}^{H(0)}\left(C_{M, N}^{\Psi}-D_{M, N}^{\Psi}\right)\right\}
\end{aligned}
$$

and (62), (66), (54) yield

$$
\begin{aligned}
& \ddot{\gamma}_{M}^{E(1)}-c^{2} \gamma_{M}^{E(1) \prime \prime}+c^{2} \beta_{M}^{2} \gamma_{M}^{E(1)} \\
& =-\frac{c^{2} \kappa_{0}(z)}{\mathcal{N}_{M}^{2}} \sum_{N}^{\prime}\left(V_{N}^{H(0) \prime}+\mu \dot{I}_{N}^{H(0)}\right) G_{M, N}^{\bar{\Phi}, \Psi}-\frac{c^{2} \kappa_{0}^{\prime}(z)}{\mathcal{N}_{M}^{2}} \sum_{N}^{\prime} V_{N}^{H(0)} G_{M, N}^{\bar{\Phi}, \Psi} \\
& -\frac{\mu c^{2} \kappa_{0}(z)}{\mathcal{N}_{M}^{2}} \sum_{N}^{\prime}\left\{\left(\beta_{N}^{2} \dot{I}_{N}^{E(0)}+\mu Y^{2} \ddot{\gamma}_{N}^{E(0)}\right) E_{M, N}^{\Phi}-\dot{I}_{N}^{E(0)}\left(C_{M, N}^{\Phi}-D_{M, N}^{\Phi}\right)\right\} \\
& +\frac{c^{2} \kappa_{0}(z)}{\mathcal{N}_{M}^{2}} \sum_{N}^{\prime}\left\{\left(\beta_{N}^{2} V_{N}^{E(0) \prime}+\gamma_{N}^{E(0) \prime \prime}\right) E_{M, N}^{\Phi}-V_{N}^{E(0) \prime}\left(C_{M, N}^{\Phi}-D_{M, N}^{\Phi}\right)\right\} \\
& -\frac{c^{2}}{\mu Y^{2} \mathcal{N}_{M}^{2}} \int_{\mathcal{D}}\left\{\rho^{(1) \prime}-\left(\rho^{(0)} \kappa_{0}^{\prime}(z)+\rho^{(0) \prime} \kappa_{0}(z)\right) r \cos \theta\right\} \overline{\Phi_{M}} \hat{\#} 1 \\
& -\frac{\mu c^{2} v}{\mathcal{N}_{M}^{2}} \dot{\rho}_{M}^{(1)}+\frac{c^{2} \kappa_{0}^{\prime}(z)}{\mathcal{N}_{M}^{2}} \sum_{N}^{\prime}\left\{\left(\beta_{N}^{2} V_{N}^{E(0)}+\gamma_{N}^{E(0) \prime}\right) E_{M, N}^{\Phi}\right. \\
& \left.\quad-V_{N}^{E(0)}\left(C_{M, N}^{\Phi}-D_{M, N}^{\Phi}\right)\right\} .
\end{aligned}
$$

The right hand sides of these expressions for $\gamma_{M}^{H(1)}$ and $\gamma_{M}^{E(1)}$ can be rewritten in terms of the lower order fields $\gamma_{N}^{E(0)}, \gamma_{N}^{H(0)}$ and the sources. Thus the following terms in (112) can be expressed as

$$
\begin{aligned}
& I_{N}^{E(0) \prime}-\mu Y^{2} \dot{V}_{N}^{E(0)}=-2\left(\frac{\mu Y^{2}}{\beta_{N}^{2}} \dot{\gamma}_{N}^{E(0) \prime}+\frac{v}{\mathcal{N}_{N}^{2} \beta_{N}^{2}} \overline{\rho_{N}^{(0) \prime}}\right), \\
& \mu Y^{2} \dot{V}_{N}^{H(0)}+I_{N}^{H(0) \prime}=\frac{1}{\alpha_{N}^{2}}\left(\frac{1}{c^{2}} \ddot{\gamma}_{N}^{H(0)}+\gamma_{N}^{H(0) \prime \prime}\right) \\
& \gamma_{N}^{H(0) \prime}+\alpha_{N}^{2} I_{N}^{H(0)}=2 \gamma_{N}^{H(0) \prime} .
\end{aligned}
$$

Similarly the following terms in (113) can be expressed as

$$
\begin{aligned}
& V_{N}^{H(0) \prime}+\mu \dot{I}_{N}^{H(0)}=2 \frac{\mu}{\alpha_{N}^{2}} \dot{\gamma}_{N}^{H(0) \prime}, \\
& \beta_{N}^{2} \dot{I}_{N}^{E(0)}+\mu Y^{2} \ddot{\gamma}_{N}^{E(0)}=\frac{v^{2}}{\mathcal{N}_{N}^{2}} \overline{\rho_{N}^{(0) \prime}}, \\
& \dot{I}_{N}^{E(0)}=-\frac{1}{\beta_{N}^{2}}\left(\mu Y^{2} \ddot{\gamma}_{N}^{E(0)}-\frac{v^{2}}{\mathcal{N}_{N}^{2}} \overline{\rho_{N}^{(0) \prime}}\right), \\
& \beta_{N}^{2} V_{N}^{E(0) \prime}+\gamma_{N}^{E(0) \prime \prime}=2 \gamma_{N}^{E(0) \prime \prime}-\frac{1}{\mathcal{N}_{N}^{2} \mu Y^{2}} \overline{\rho_{N}^{(0) \prime}}, \\
& V_{N}^{E(0) \prime}=\frac{1}{\beta_{N}^{2}}\left(\gamma_{N}^{E(0) \prime \prime}-\frac{1}{\mu Y^{2}} \overline{\rho_{N}^{(0) \prime}}\right) .
\end{aligned}
$$


Finally once $\gamma_{M}^{H(1)}$ and $\gamma_{M}^{E(1)}$ have been determined from these decoupled equations it follows from (66), (56), (62), (64) that the remaining fields can be readily determined:

$$
\begin{aligned}
V_{M}^{E(1)}= & \frac{1}{\beta_{M}^{2}}\left[\gamma_{M}^{E(1) \prime}-\frac{1}{\mu Y^{2} \mathcal{N}_{M}^{2}} \int_{\mathcal{D}}\left(\rho^{(1)}-\rho^{(0)} \kappa_{0}(z) r \cos \theta\right) \overline{\Phi_{M}} \hat{\#} 1\right. \\
& \left.+\frac{\kappa_{0}(z)}{\mathcal{N}_{M}^{2}} \sum_{N}^{\prime}\left\{\left(V_{N}^{E(0)} \beta_{N}^{2}+\gamma_{N}^{E(0) \prime}\right) E_{M, N}^{\Phi}-V_{N}^{E(0)}\left(C_{M, N}^{\Phi}-D_{M, N}^{\Phi}\right)\right\}\right], \\
V_{M}^{H(1)}= & \frac{1}{\alpha_{M}^{2}}\left\{\mu \dot{\gamma}_{M}^{H(1)}+\frac{\kappa_{0}(z)}{\mathcal{M}_{M}^{2}} \sum_{N}^{\prime} V_{N}^{H(0)}\left(C_{M, N}^{\Psi}-D_{M, N}^{\Psi}\right)\right\}, \\
I_{M}^{E(1)}= & -\frac{1}{\beta_{M}^{2}}\left[\mu Y^{2} \dot{\gamma}_{M}^{E(1)}+\frac{\kappa_{0}(z)}{\mathcal{N}_{M}^{2}} \sum_{N}^{\prime}\left\{\left(\beta_{N}^{2} I_{N}^{E(0)}+\mu Y^{2} \dot{\gamma}_{N}^{E(0)}\right) E_{M, N}^{\Phi}\right.\right. \\
& \left.\left.-I_{N}^{E(0)}\left(C_{M, N}^{\Phi}-D_{M, N}^{\Phi}\right)\right\}+\frac{v}{\mathcal{N}_{M}^{2}} \overline{\rho_{M}^{(1)}}\right], \\
I_{M}^{H(1)}= & \frac{1}{\alpha_{M}^{2}}\left[\gamma_{M}^{H(1) \prime}+\frac{\kappa_{0}(z)}{\mathcal{M}_{M}^{2}} \sum_{N}^{\prime}\left\{\left(\gamma_{N}^{H(0) \prime}+\alpha_{N}^{2} I_{N}^{H(0)}\right) E_{M, N}^{\Psi}\right.\right. \\
& \left.\left.-I_{N}^{H(0)}\left(C_{M, N}^{\Psi}-D_{M, N}^{\Psi}\right)\right\}\right] .
\end{aligned}
$$

The distributional nature of the source permits evaluation of the integrals in (112) and (113) yielding

$$
\begin{aligned}
& \int_{\mathcal{D}}\left\{\rho^{(1) \prime}-\left(\rho^{(0)} \kappa_{0}^{\prime}(z)+\rho^{(0) \prime} \kappa_{0}(z)\right) r \cos \theta\right\} \overline{\Phi_{M}} \hat{\#} 1 \\
& =r_{0} \cos \theta_{0} Q_{t o t} J_{m}\left(x_{p(m)} \frac{r_{0}}{a}\right) e^{-i m \theta_{0}} \\
& \times\left\{\delta^{\prime}(z-v t)\left(\kappa_{0}(v t)-\kappa_{0}(z)\right)-\delta(z-v t) \kappa_{0}^{\prime}(z)\right\},
\end{aligned}
$$

and

$$
\begin{aligned}
v \int_{\mathcal{D}} \dot{\rho}^{(1)} \overline{\Phi_{M}} \hat{\#} 1= & v^{2} x_{1,0} Q_{t o t} J_{m}\left(x_{p(m)} \frac{r_{0}}{a}\right) e^{-i m \theta_{0}} \\
& \times\left(-\kappa_{0}(v t) \delta^{\prime}(z-v t)+\kappa_{0}^{\prime}(v t) \delta(z-v t)\right)
\end{aligned}
$$

where $\kappa_{0}^{\prime}(v t)=d \kappa_{0}(z) /\left.d z\right|_{z=v t}$.

The solutions for $\gamma_{M}^{H(1)}$ and $\gamma_{M}^{E(1)}$ follow similarly from (76). The source term in the equation for $\gamma_{M}^{H(1)}$ is

$$
g_{M}^{H(1)}(t, z)=K_{M}^{H(1)}(t, z)+L_{M}^{H(1)}(t, z)
$$

where, for $\kappa_{0}$ varying with $z,[18$

$$
\begin{aligned}
K_{M}^{H(1)}(t, z) & :=\frac{c^{2} \kappa_{0}(z)}{\mathcal{M}_{M}^{2}} \sum_{N}^{\prime}\left\{2 G_{M, N}^{\bar{\Psi}, \Phi} \frac{\mu Y^{2}}{\beta_{N}^{2}} \dot{\gamma}_{N}^{E(0) \prime}\right. \\
- & \left.\frac{C_{M, N}^{\Psi}-D_{M, N}^{\Psi}}{\alpha_{N}^{2}}\left(\frac{\ddot{\gamma}_{N}^{H(0)}}{c^{2}}+\gamma_{N}^{H(0) \prime \prime}\right)+2 E_{M, N}^{\Psi} \gamma_{N}^{H(0) \prime \prime}\right\} \\
+ & \frac{c^{2} \kappa_{0}^{\prime}(z)}{\mathcal{M}_{M}^{2}} \sum_{N}^{\prime}\left\{\frac{\mu Y^{2}}{\beta_{N}^{2}} G_{M, N}^{\bar{\Psi}, \Phi} \dot{\gamma}_{N}^{E(0)}\right. \\
+ & \left.\left(2 E_{M, N}^{\Psi}-\frac{C_{M, N}^{\Psi}-D_{M, N}^{\Psi}}{\alpha_{N}^{2}}\right) \gamma_{N}^{H(0) \prime}\right\} \\
L_{M}^{H(1)}(t, z) & :=\frac{2 c^{2} v Q_{t o t}}{\mathcal{M}_{M}^{2}} \kappa_{0}(z) \delta^{\prime}(z-v t) \sum_{N}^{\prime} \frac{G_{M, N}^{\bar{\Psi}, \Phi}}{\mathcal{N}_{N}^{2} \beta_{N}^{2}} J_{n}\left(x_{q(n)} \frac{r_{0}}{a}\right) e^{-i n \theta_{0}} \\
S_{M}^{H(1)}(t, z) & :=\frac{c^{2} v Q_{t o t}}{\mathcal{M}_{M}^{2}} \kappa_{0}^{\prime}(v t) \delta(z-v t) \sum_{N}^{\prime} \frac{G_{M, N}^{\bar{\Psi}, \Phi}}{\mathcal{N}_{N}^{2} \beta_{N}^{2}} J_{n}\left(x_{q(n)} \frac{r_{0}}{a}\right) e^{-i n \theta_{0}}
\end{aligned}
$$


The source term in the equation for $\gamma_{M}^{E(1)}$ is

$$
g_{M}^{E(1)}(t, z)=K_{M}^{E(1)}(t, z)+L_{M}^{E(1)}(t, z)+P_{M}^{E(1)}(t, z)+R_{M}^{E(1)}(t, z)+S_{M}^{E(1)}(t, z),
$$

where [19]

$$
\begin{aligned}
& K_{M}^{E(1)}(t, z):=\frac{c^{2} \kappa_{0}(z)}{\mathcal{N}_{M}^{2}} \sum_{N}^{\prime}\left\{\frac{-2 \mu}{\alpha_{N}^{2}} G_{M, N}^{\bar{\Phi}, \Psi} \dot{\gamma}_{N}^{H(0) \prime}-\frac{C_{M, N}^{\Phi}-D_{M, N}^{\Phi}}{c^{2} \beta_{N}^{2}} \ddot{\gamma}_{N}^{E(0)}\right. \\
& \left.+\left(2 E_{M, N}^{\Phi}-\frac{C_{M, N}^{\Phi}-D_{M, N}^{\Phi}}{\beta_{N}^{2}}\right) \gamma_{N}^{E(0) \prime \prime}\right\} \\
& +\frac{c^{2} \kappa_{0}^{\prime}(z)}{\mathcal{N}_{M}^{2}} \sum_{N}^{\prime}\left\{\frac{-\mu}{\alpha_{N}^{2}} G_{M, N}^{\bar{\Phi}, \Psi} \dot{\gamma}_{N}^{H(0)}\right. \\
& \left.+\left(2 E_{M, N}^{\Phi}-\frac{C_{M, N}^{\Phi}-D_{M, N}^{\Phi}}{\beta_{N}^{2}}\right) \gamma_{N}^{E(0) \prime}\right\} \\
& L_{M}^{E(1)}(t, z):=\frac{\mu c^{2}\left(c^{2}+v^{2}\right) Q_{t o t}}{\mathcal{N}_{M}^{2}} \kappa_{0}(z) \delta^{\prime}(z-v t) \\
& \times \sum_{N}^{\prime} \frac{1}{\mathcal{N}_{N}^{2}}\left(-E_{M, N}^{\Phi}+\frac{C_{M, N}^{\Phi}-D_{M, N}^{\Phi}}{\beta_{N}^{2}}\right) J_{n}\left(x_{q(n)} \frac{r_{0}}{a}\right) e^{-i n \theta_{0}}, \\
& P_{M}^{E(1)}(t, z):=-\frac{\mu c^{2} v^{2} Q_{t o t}}{\mathcal{N}_{M}^{2}} r_{0} \cos \theta_{0} J_{m}\left(x_{p(m)} \frac{r_{0}}{a}\right) e^{-i m \theta_{0}} \\
& \times\left\{\kappa_{0}^{\prime}(v t) \delta(z-v t)-\kappa_{0}(v t) \delta^{\prime}(z-v t)\right\}, \\
& R_{M}^{E(1)}(t, z):=-\frac{\mu c^{4} Q_{t o t}}{\mathcal{N}_{M}^{2}} r_{0} \cos \theta_{0} J_{m}\left(x_{p(m)} \frac{r_{0}}{a}\right) e^{-i m \theta_{0}} \\
& \times\left(\delta^{\prime}(z-v t)\left\{\kappa_{0}(v t)-\kappa_{0}(z)\right\}-\delta(z-v t) \kappa_{0}^{\prime}(z)\right), \\
& S_{M}^{E(1)}(t, z):=\frac{\mu c^{4} Q_{t o t}}{\mathcal{N}_{M}^{2}} \kappa_{0}^{\prime}(z) \delta(z-v t) \\
& \times \sum_{N}^{\prime} \frac{1}{\mathcal{N}_{N}^{2}}\left(-E_{M, N}^{\Phi}+\frac{C_{M, N}^{\Phi}-D_{M, N}^{\Phi}}{\beta_{N}^{2}}\right) J_{n}\left(x_{q(n)} \frac{r_{0}}{a}\right) e^{-i n \theta_{0}} .
\end{aligned}
$$

In the next subsection, $\$ \mathrm{VIIC}$, the fields associated with an ultra-relativistic point source will be of interest. Then the contributions to the solution $\gamma_{N}^{E(0)}$ that depend on $Q_{t o t}$ tend to zero and the solution $\gamma_{N}^{H(0)}$ depends only on external and static magnetic fields since there are no magnetic charges in existence. In this limit the source contributions $K_{M}^{H(1)}$ and $K_{M}^{E(1)}$ to $g_{M}^{H(1)}$ and $g_{M}^{E(1)}$ drop out.

In general, an analytic form for $\gamma_{M}^{H(1)}$ follows from (77) and (78), by applying the integral operators $\mathcal{H}_{\alpha_{M}}$ and $\mathcal{I}_{\alpha_{M}}$ to the source functions $K_{M}^{E(1)}, L_{M}^{E(1)}, P_{M}^{E(1)}, R_{M}^{E(1)}, S_{M}^{E(1)}$. These source functions are simple functions of $z, t$ multiplied by (complex) numerical coefficients. Thus with

$$
\begin{aligned}
& f_{1}(t, z):=\kappa_{0}(z) \delta^{\prime}(z-v t) \\
& f_{2}(t, z):=\kappa_{0}^{\prime}(v t) \delta(z-v t) \\
& f_{3}(t, z):=\kappa_{0}(v t) \delta^{\prime}(z-v t)
\end{aligned}
$$

we write the application of $\mathcal{I}_{\sigma}$ on them as

$$
\begin{aligned}
& \mathcal{I}_{\sigma}\left[f_{1}\right](t, z)=-\mathcal{A}_{\sigma}(t, z)+\mathcal{B}_{\sigma}(t, z), \\
& \mathcal{I}_{\sigma}\left[f_{2}\right](t, z)=\mathcal{A}_{\sigma}(t, z), \\
& \mathcal{I}_{\sigma}\left[f_{3}\right](t, z)=\mathcal{B}_{\sigma}(t, z),
\end{aligned}
$$

where $\mathcal{A}_{\sigma}$ and $\mathcal{B}_{\sigma}$, are calculated to be (See Fig \): 
- for $z \leq-c t$, (Outside the regions $R_{1}$ and $R_{2}$ )

$$
\mathcal{A}_{\sigma}(t, z):=0, \quad \mathcal{B}_{\sigma}(t, z):=0,
$$

- for $-c t<z \leq v t$, (Region $\left.R_{2}\right)$

$$
\begin{array}{r}
\mathcal{A}_{\sigma}(t, z):=\frac{1}{2 c} \int_{0}^{t_{+}^{\prime}(t, z)} d t^{\prime} \kappa_{0}^{\prime}\left(v t^{\prime}\right) J_{0}\left(\sigma s\left(t, z \mid t^{\prime}\right)\right), \\
\mathcal{B}_{\sigma}(t, z):=\frac{\sigma}{2 c} \int_{0}^{t_{+}^{\prime}(t, z)} d t^{\prime} \frac{\kappa_{0}\left(v t^{\prime}\right)\left(z-v t^{\prime}\right)}{s\left(t, z \mid t^{\prime}\right)} J_{1}\left(\sigma s\left(t, z \mid t^{\prime}\right)\right),
\end{array}
$$

- for $v t \leq z<c t,\left(\operatorname{Region} R_{1}\right)$

$$
\begin{array}{r}
\mathcal{A}_{\sigma}(t, z):=\frac{1}{2 c} \int_{0}^{t_{-}^{\prime}(t, z)} d t^{\prime} \kappa_{0}^{\prime}\left(v t^{\prime}\right) J_{0}\left(\sigma s\left(t, z \mid t^{\prime}\right)\right), \\
\mathcal{B}_{\sigma}(t, z):=\frac{\sigma}{2 c} \int_{0}^{t_{-}^{\prime}(t, z)} d t^{\prime} \frac{\kappa_{0}\left(v t^{\prime}\right)\left(z-v t^{\prime}\right)}{s\left(t, z \mid t^{\prime}\right)} J_{1}\left(\sigma s\left(t, z \mid t^{\prime}\right)\right),
\end{array}
$$

- for $z \geq c t$, (Outside the regions $R_{1}$ and $R_{2}$ )

$$
\mathcal{A}_{\sigma}(t, z):=0, \quad \mathcal{B}_{\sigma}(t, z):=0,
$$

with $t_{+}^{\prime}(t, z), t_{-}^{\prime}(t, z)$ and $s\left(t, z \mid t^{\prime}\right)$ defined in (110). Thus one sees that to $\mathcal{O}(\epsilon), \mathcal{A}_{\sigma}$ containes $\kappa_{0}^{\prime}$ while $\mathcal{B}_{\sigma}$ containes $\kappa_{0}$.

The causal solution for $\gamma_{M}^{H(1)}$ generated by the source term $g_{M}^{H(1)}$ above then follows from (78) and can be written:

$$
\begin{aligned}
\gamma_{M}^{H(1)}(t, z) & =\mathcal{I}_{\alpha_{M}}\left[g_{M}^{H(1)}\right](t, z) \\
& =\mathcal{I}_{\alpha_{M}}\left[K_{M}^{H(1)}\right](t, z)+\mathcal{I}_{\alpha_{M}}\left[L_{M}^{H(1)}\right](t, z)+\mathcal{I}_{\alpha_{M}}\left[S_{M}^{H(1)}\right](t, z) .
\end{aligned}
$$

The first term on the right above depends on initial data but explicit expressions for the last two terms are:

$$
\begin{aligned}
\mathcal{I}_{\alpha_{M}}\left[L_{M}^{H(1)}\right](t, z)= & \frac{2 c^{2} v Q_{t o t}}{\mathcal{M}_{M}^{2}}\left(-\mathcal{A}_{\alpha_{M}}(t, z)+\mathcal{B}_{\alpha_{M}}(t, z)\right) \\
& \times \sum_{N}^{\prime} \frac{G_{M, N}^{\bar{\Psi}, \Phi}}{\mathcal{N}_{N}^{2} \beta_{N}^{2}} J_{n}\left(x_{q(n)} \frac{r_{0}}{a}\right) e^{-i n \theta_{0}},
\end{aligned}
$$

and

$$
\begin{aligned}
\mathcal{I}_{\alpha_{M}}\left[S_{M}^{H(1)}\right](t, z)=\frac{c^{2} v Q_{t o t}}{\mathcal{M}_{M}^{2}} \mathcal{A}_{\alpha_{M}}(t, z) & \\
& \times \sum_{N}^{\prime} \frac{G_{M, N}^{\bar{\Psi}, \Phi}}{\mathcal{N}_{N}^{2} \beta_{N}^{2}} J_{n}\left(x_{q(n)} \frac{r_{0}}{a}\right) e^{-i n \theta_{0}} .
\end{aligned}
$$

Similarly the causal solution for $\gamma_{M}^{E(1)}$ generated by the source term $g_{M}^{E(1)}$ can be written as:

$$
\begin{aligned}
\gamma_{M}^{E(1)}(t, z)= & \mathcal{I}_{\beta_{M}}\left[g_{M}^{E(1)}\right](t, z) \\
= & \mathcal{I}_{\beta_{M}}\left[K_{M}^{E(1)}\right](t, z)+\mathcal{I}_{\beta_{M}}\left[L_{M}^{E(1)}\right](t, z)+\mathcal{I}_{\beta_{M}}\left[P_{M}^{E(1)}\right](t, z) \\
& +\mathcal{I}_{\beta_{M}}\left[R_{M}^{E(1)}\right](t, z)+\mathcal{I}_{\beta_{M}}\left[S_{M}^{E(1)}\right],
\end{aligned}
$$

with

$$
\begin{aligned}
& \mathcal{I}_{\beta_{M}}\left[L_{M}^{E(1)}\right](t, z)=\frac{\mu c^{2}\left(c^{2}+v^{2}\right) Q_{t o t}}{\mathcal{N}_{M}^{2}}\left(-\mathcal{A}_{\beta_{M}}(t, z)+\mathcal{B}_{\beta_{M}}(t, z)\right) \\
& \times \sum_{N}^{\prime} \frac{1}{\mathcal{N}_{N}^{2}}\left(-E_{M, N}^{\Phi}+\frac{C_{M, N}^{\Phi}-D_{M, N}^{\Phi}}{\beta_{N}^{2}}\right) J_{n}\left(x_{q(n)} \frac{r_{0}}{a}\right) e^{-i n \theta_{0}}
\end{aligned}
$$




$$
\begin{gathered}
\mathcal{I}_{\beta_{M}}\left[P_{M}^{E(1)}\right](t, z)=-\frac{\mu c^{2} v^{2} Q_{t o t}}{\mathcal{N}_{M}^{2}}\left(\mathcal{A}_{\beta_{M}}(t, z)-\mathcal{B}_{\beta_{M}}(t, z)\right) \\
\quad \times r_{0} \cos \theta_{0} J_{m}\left(x_{p(m)} \frac{r_{0}}{a}\right) e^{-i m \theta_{0}}, \\
\mathcal{I}_{\beta_{M}}\left[R_{M}^{E(1)}\right](t, z)=0, \\
\mathcal{I}_{\beta_{M}}\left[S_{M}^{E(1)}\right](t, z)=\frac{\mu c^{4} Q_{t o t}}{\mathcal{N}_{M}^{2}} \mathcal{A}_{\beta_{M}}(t, z) \\
\times \sum_{N}^{\prime} \frac{1}{\mathcal{N}_{N}^{2}}\left(-E_{M, N}^{\Phi}+\frac{C_{M, N}^{\Phi}-D_{M, N}^{\Phi}}{\beta_{N}^{2}}\right) J_{n}\left(x_{q(n)} \frac{r_{0}}{a}\right) e^{-i n \theta_{0}} .
\end{gathered}
$$

The contribution $\mathcal{I}_{\beta_{M}}\left[K_{M}^{E(1)}\right](t, z)$ depends on initial data and electric currents that vanish in the ultra-relativistic limit.

\section{First Order Contribution to the Instantaneous Power from a Moving Point Charge}

In terms of the lowest order modal solutions the instantaneous power $\breve{w}^{(1)}(t, z)$ for $z \neq v t, t>0$ can now be computed to $\mathcal{O}\left(\epsilon^{1}\right)$ from (84):

$$
\begin{aligned}
& \breve{w}^{(1)}=-\Re\left[\mu Y^{2} \sum_{N} \frac{\mathcal{N}_{N}^{2}}{\beta_{N}^{2}} \gamma_{N}^{E(0) \prime}\left(\overline{\dot{\gamma}_{N}^{E(1)}}+\frac{\kappa_{0}(z)}{\mathcal{N}_{N}^{2}} \sum_{M}^{\prime} \frac{C_{N, M}^{\Phi}-D_{N, M}^{\Phi}}{\beta_{M}^{2}} \overline{\dot{\gamma}_{M}^{E(0)}}\right)\right. \\
& +\mu Y^{2} \sum_{N} \frac{\mathcal{N}_{N}^{2}}{\beta_{N}^{2}}\left\{\gamma_{N}^{E(1) \prime}+\frac{\kappa_{0}(z)}{\mathcal{N}_{N}^{2}} \sum_{M}^{\prime}\left(2 E_{N, M}^{\Phi}-\frac{C_{M, N}^{\Phi}-D_{M, N}^{\Phi}}{\beta_{M}^{2}}\right) \gamma_{M}^{E(0) \prime}\right\} \overline{\dot{\gamma}_{N}^{E(0)}} \\
& +\mu \sum_{M} \frac{\mathcal{M}_{M}^{2}}{\alpha_{M}^{2}} \dot{\gamma}_{M}^{H(0)}\left\{\overline{\gamma_{M}^{H(1) \prime}}+\frac{\kappa_{0}(z)}{\mathcal{M}_{M}^{2}} \sum_{N}^{\prime}\left(2 E_{M, N}^{\Psi}-\frac{C_{M, N}^{\Psi}-D_{M, N}^{\Psi}}{\alpha_{N}^{2}}\right) \overline{\gamma_{N}^{H(0) \prime}}\right\} \\
& +\mu \sum_{M} \frac{\mathcal{M}_{M}^{2}}{\alpha_{M}^{2}}\left(\dot{\gamma}_{M}^{H(1)}+\frac{\kappa_{0}(z)}{\mathcal{M}_{M}^{2}} \sum_{N}^{\prime} \frac{C_{M, N}^{\Psi}-D_{M, N}^{\Psi}}{\alpha_{N}^{2}} \dot{\gamma}_{N}^{H(0)}\right) \overline{\gamma_{M}^{H(0) \prime}} \\
& \left.+\kappa_{0}(z) \mu Y^{2} \sum_{N} \sum_{N^{\prime}} F_{N, N^{\prime}}^{\Phi} \frac{\gamma_{N}^{E(0) \prime} \dot{\gamma}_{N^{\prime}}^{E(0)}}{\beta_{N}^{2} \beta_{N^{\prime}}^{2}}+\kappa_{0}(z) \mu \sum_{M} \sum_{M^{\prime}} F_{M, M^{\prime}}^{\Psi} \frac{\dot{\gamma}_{M}^{H(0)} \overline{\gamma_{M^{\prime}}^{H(0) \prime}}}{\alpha_{M^{2}}^{2} \alpha_{M^{\prime}}^{2}}\right] .
\end{aligned}
$$

\section{Ultra-relativistic Longitudinal Wake Potentials}

The wakefield formalism is designed to exploit the simplifications that arise by considering the unphysical (ultrarelativistic) limit obtained from charged sources moving at the speed of light. The resulting electromagnetic fields give rise to various wake-potentials from which wake-impedances may be computed for ultra-relativistic charged bunches with prescribed charged distributions. The formalism is based on calculating the emf induced on a spectator (test) ultra-relativistic point particle moving behind a leading ultra-relativistic charged particle with the same velocity but in general on a different orbit. Since the section above provides the electromagnetic fields for a point particle moving with arbitrary speed on an orbit (in general) off the tube axis (with transverse coordinates $\left(r_{0}, \theta_{0}\right)$ ) one may readily calculate the general longitudinal wake potential to the same order as the fields, by having the spectator charge, with transverse coordinates $(r, \theta)$, at a fixed longitudinal separation $\widetilde{s}>0$ behind a right moving source particle.

The definition [13] of the ultra-relativistic longitudinal wake potential is taken as

$$
\mathcal{W}_{\|}^{\left(r_{0}, \theta_{0}\right)}(\epsilon, r, \theta, \widetilde{s}):=-\frac{1}{Q_{t o t}} \int_{-\widetilde{s} / 2}^{\infty} d z \mathcal{E}_{z}^{\left(r_{0}, \theta_{0}\right)}\left(\epsilon, \frac{z+\widetilde{s}}{c}, z, r, \theta\right)
$$


where $\mathcal{E}_{z}^{\left(r_{0}, \theta_{0}\right)}(\epsilon, t, z, r, \theta)$ is the $z$-component of the electric field generated by the point source with speed $v=c$ and charge $Q_{t o t} .20$. Since the $z$-component of the total electric field is

$$
\begin{aligned}
& i_{\partial_{z}}^{\mathbf{e}}(1) \\
&(\epsilon, t, z, r, \theta)=\sum_{N} \gamma_{N}^{E}(\epsilon, t, z) \Phi_{N}(r, \theta) \\
&=\epsilon \sum_{N} \gamma_{N}^{E(1)}(t, z) \Phi_{N}(r, \theta)+\mathcal{O}\left(\epsilon^{2}\right),
\end{aligned}
$$

one has

$$
\mathcal{E}_{z}^{\left(r_{0}, \theta_{0}\right)}(\epsilon, t, z, r, \theta)=\epsilon \sum_{M} \breve{\gamma}_{M}^{E(1)}(t, z) \Phi_{M}(r, \theta)+\mathcal{O}\left(\epsilon^{2}\right)
$$

with

$$
\breve{\gamma}_{M}^{E(1)}(t, z):=\gamma_{M}^{E(1)}(t, z)-\mathcal{I}_{\beta_{M}}\left[K_{M}^{E(1)}\right](t, z)
$$

Thus,

$$
\mathcal{W}_{\|}^{\left(r_{0}, \theta_{0}\right)}(\epsilon, r, \theta, \widetilde{s})=-\frac{\epsilon}{Q_{t o t}} \sum_{M} \int_{-\widetilde{s} / 2}^{\infty} d z \breve{\gamma}_{M}^{E(1)}\left(\frac{z+\widetilde{s}}{c}, z\right) \Phi_{M}(r, \theta)+\mathcal{O}\left(\epsilon^{2}\right)
$$

From the orthogonality relation (34), one calculates the projected longitudinal wake potentials:

$$
\mathcal{W}_{\|}^{\left(r_{0}, \theta_{0}\right)}(\epsilon, \widetilde{s}):=\int_{\mathcal{D}} \mathcal{W}_{\|}^{\left(r_{0}, \theta_{0}\right)} \Phi_{M} \hat{\#} 1
$$

Hence

$$
\mathcal{W}_{\|}^{\left(r_{0}, \theta_{0}\right)}(\epsilon, \widetilde{s})=-\epsilon \frac{\mathcal{N}_{M}^{2}}{Q_{t o t}} \int_{-\widetilde{s} / 2}^{\infty} d z \overline{\breve{\gamma}_{M}^{E(1)}}\left(\frac{z+\widetilde{s}}{c}, z\right)+\mathcal{O}\left(\epsilon^{2}\right)
$$

From (125), the definition of the ultra-relativistic longitudinal impedance is taken as

$$
Z_{\|}^{\left(r_{0}, \theta_{0}\right)}(\epsilon, r, \theta, \omega):=\frac{1}{c} \int_{0}^{\infty} d \widetilde{s} e^{i \omega \widetilde{s} / c} \mathcal{W}_{\|}^{\left(r_{0}, \theta_{0}\right)}(\epsilon, r, \theta, \widetilde{s}),
$$

and from the orthogonality relation (34), one calculates the projected longitudinal impedances:

$$
\left\langle Z_{\|}^{\left(r_{0}, \theta_{0}\right)}\right\rangle_{M}(\epsilon, \omega):=\int_{\mathcal{D}} Z_{\|}^{\left(r_{0}, \theta_{0}\right)}(\epsilon, r, \theta, \omega) \overline{\Phi_{M}}(r, \theta) \hat{\#} 1 .
$$

To calculate (128), one needs $\breve{\gamma}_{M}^{E(1)}(t, z)$. In the ultra-relativistic limit the expressions (116), (117), (118) become

$$
\begin{aligned}
& \check{f}_{1}(t, z):=\kappa_{0}(z) \delta^{\prime}(z-c t), \\
& \check{f}_{2}(t, z):=\kappa_{0}^{\prime}(c t) \delta(z-c t), \\
& \check{f}_{3}(t, z):=\kappa_{0}(c t) \delta^{\prime}(z-c t) .
\end{aligned}
$$

One may write the application of the integral operator $\mathcal{I}_{\sigma}$ on these as

$$
\begin{aligned}
& \mathcal{I}_{\sigma}\left[\check{f}_{1}\right](t, z)=-\check{\mathcal{A}}_{\sigma}(t, z)+\check{\mathcal{B}}_{\sigma}(t, z), \\
& \mathcal{I}_{\sigma}\left[\check{f}_{2}\right](t, z)=\check{\mathcal{A}}_{\sigma}(t, z), \\
& \mathcal{I}_{\sigma}\left[\check{f}_{3}\right](t, z)=\check{\mathcal{B}}_{\sigma}(t, z) .
\end{aligned}
$$

where $\check{\mathcal{A}}_{\sigma}$ and $\check{\mathcal{B}}_{\sigma}$ are given in the following domains:

- for $z \leq-c t$,

$$
\check{\mathcal{A}}_{\sigma}(t, z):=0, \quad \check{\mathcal{B}}_{\sigma}(t, z):=0,
$$


- for $-c t<z<c t$,

$$
\begin{array}{r}
\check{\mathcal{A}}_{\sigma}(t, z):=\frac{1}{2 c} \int_{0}^{t_{c}^{\prime}(t, z)} d t^{\prime} \kappa_{0}^{\prime}\left(c t^{\prime}\right) J_{0}\left(\sigma s_{c}\left(t, z \mid t^{\prime}\right)\right), \\
\check{\mathcal{B}}_{\sigma}(t, z):=\frac{\sigma}{2 c} \int_{0}^{t_{c}^{\prime}(t, z)} d t^{\prime} \frac{\kappa_{0}\left(c t^{\prime}\right)\left(z-c t^{\prime}\right)}{s_{c}\left(t, z \mid t^{\prime}\right)} J_{1}\left(\sigma s_{c}\left(t, z \mid t^{\prime}\right)\right),
\end{array}
$$

- for $z=c t$,

$$
\begin{array}{r}
\check{\mathcal{A}}_{\sigma}(t, z):=\frac{1}{4 c} \int_{0}^{t} d t^{\prime} \kappa_{0}^{\prime}\left(c t^{\prime}\right) J_{0}\left(\sigma s_{c}\left(t, z \mid t^{\prime}\right)\right), \\
\check{\mathcal{B}}_{\sigma}(t, z):=\frac{\sigma}{4 c} \int_{0}^{t} d t^{\prime} \frac{\kappa_{0}\left(c t^{\prime}\right)\left(z-c t^{\prime}\right)}{s_{c}\left(t, z \mid t^{\prime}\right)} J_{1}\left(\sigma s_{c}\left(t, z \mid t^{\prime}\right)\right),
\end{array}
$$

- for $z \geq c t$,

$$
\check{\mathcal{A}}_{\sigma}(t, z):=0, \quad \check{\mathcal{B}}_{\sigma}(t, z):=0,
$$

where

$$
s_{c}\left(t, z \mid t^{\prime}\right):=\sqrt{c^{2}\left(t-t^{\prime}\right)^{2}-\left(z-c t^{\prime}\right)^{2}}
$$

and

$$
t_{c}^{\prime}(t, z):=\frac{1}{2}\left(t+\frac{z}{c}\right)
$$

It immediately follows that

$$
s_{c}\left(t, c t \mid t^{\prime}\right)=0 .
$$

The explicit form of $\check{\mathcal{A}}_{\sigma}(t, z)$ and $\check{\mathcal{B}}_{\sigma}(t, z)$ follows from a calculation similar to that outlined for $\mathcal{A}_{\sigma}(t, z)$ and $\mathcal{B}_{\sigma}(t, z)$ respectively in Appendix A. Thus $\check{\mathcal{A}}_{\sigma}$ and $\check{\mathcal{B}}_{\sigma}$ correspond to $\mathcal{A}_{\sigma}$ and $\mathcal{B}_{\sigma}$ respectively in the case when $0<v<c$.

Using the results in $\overline{\mathrm{VIIB}}$ and (132), (134), (134), (135) one finds

$$
\begin{aligned}
& \breve{\gamma}_{M}^{E(1)}(t, z)=\mathcal{I}_{\beta_{M}}\left[L_{M}^{E(1)}+P_{M}^{E(1)}+R_{M}^{E(1)}+S_{M}^{E(1)}\right](t, z), \\
& =l_{M}^{\left(r_{0}, \theta_{0}\right)}\left(-\check{\mathcal{A}}_{\beta_{M}}+\check{\mathcal{B}}_{\beta_{M}}\right)+p_{M}^{\left(r_{0}, \theta_{0}\right)}\left(\check{\mathcal{A}}_{\beta_{M}}-\check{\mathcal{B}}_{\beta_{M}}\right)+s_{M}^{\left(r_{0}, \theta_{0}\right)} \check{\mathcal{A}}_{\beta_{M}} \\
& =\left(-l_{M}^{\left(r_{0}, \theta_{0}\right)}+p_{M}^{\left(r_{0}, \theta_{0}\right)}+s_{M}^{\left(r_{0}, \theta_{0}\right)}\right) \check{\mathcal{A}}_{\beta_{M}}+\left(l_{M}^{\left(r_{0}, \theta_{0}\right)}-p_{M}^{\left(r_{0}, \theta_{0}\right)}\right) \check{\mathcal{B}}_{\beta_{M}},
\end{aligned}
$$

where

$$
\begin{aligned}
& l_{M}^{\left(r_{0}, \theta_{0}\right)}:=\frac{2 \mu c^{4}}{\mathcal{N}_{M}^{2}} Q_{t o t} \sum_{N}^{\prime} \frac{1}{\mathcal{N}_{N}^{2}}\left(-E_{M, N}^{\Phi}+\frac{C_{M, N}^{\Phi}-D_{M, N}^{\Phi}}{\beta_{N}^{2}}\right) \\
& \times J_{n}\left(x_{q(n)} \frac{r_{0}}{a}\right) e^{-i n \theta_{0}}, \\
& p_{M}^{\left(r_{0}, \theta_{0}\right)}:=\frac{\mu c^{4}}{\mathcal{N}_{M}^{2}} Q_{t o t} r_{0} \cos \theta_{0} J_{m}\left(x_{p(m)} \frac{r_{0}}{a}\right) e^{-i m \theta_{0}}, \\
& s_{M}^{\left(r_{0}, \theta_{0}\right)}:=\frac{\mu c^{4}}{\mathcal{N}_{M}^{2}} Q_{t o t} \sum_{N}^{\prime} \frac{1}{\mathcal{N}_{N}^{2}}\left(-E_{M, N}^{\Phi}+\frac{C_{M, N}^{\Phi}-D_{M, N}^{\Phi}}{\beta_{N}^{2}}\right) \\
& \times J_{n}\left(x_{q(n)} \frac{r_{0}}{a}\right) e^{-i n \theta_{0}} .
\end{aligned}
$$

From these expressions one calculates (128) and (127). Thus $\breve{\gamma}_{M}^{E(1)}$ and hence (128) can be expressed in terms of separate contributions from $\kappa_{0}(z)$ and $\kappa_{0}^{\prime}(z)$. 


\section{Longitudinal Wake Potential for a Pipe with Piecewise Constant Curvature}

In the last section explicit formulae are given for the computation to leading order of the longitudinal wake potential in a pipe with arbitrary smooth curvature and $|\kappa(z) a|<1$. From such potentials one may calculate the longitudinal impedance to the same order. These expressions involve integrals of the curvature with Bessel functions and such integrals in general require numerical analysis. However in cases where segments of the beam pipe are connected by planar segments of arcs with constant radius of curvature (See Fig 2) one may perform these integrals analytically and hence generate analytic expressions for the corresponding wake impedances. In principle there is an element of further approximation involved if one assumes that the tangent to the axial space-curve is discontinuous where the straight segment joins the curved segment. However bearing this in mind consider the case of an infinitely long planar pipe with axial curvature given by

$$
\kappa_{0}(z)=\left(\Theta\left(z-z_{L}\right)-\Theta\left(z-z_{R}\right)\right) \check{\kappa}_{0},
$$

where $z_{L}, z_{R},\left(0<z_{L}<z_{R}\right), \check{\kappa}_{0}(\neq 0)$ are constants and $\Theta(z)$ is the Heaviside function

$$
\Theta(z)= \begin{cases}1, & \text { for } z \geq 0 \\ 0, & \text { otherwise }\end{cases}
$$

In this case one can calculate $\check{\mathcal{A}}_{\sigma}, \check{\mathcal{B}}_{\sigma}$ and write (128) in terms of known functions.

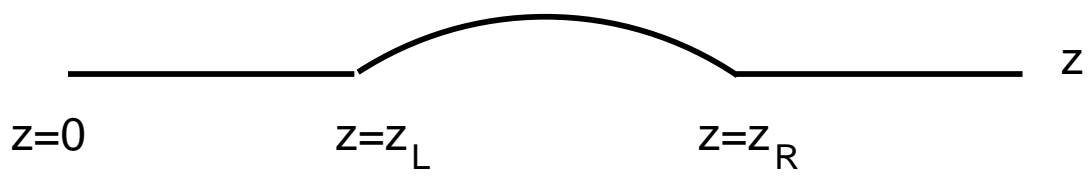

FIG. 2: Profile of beam pipe with a segment of constant curvature.

One finds that the terms in the wake potential proportional to $\check{\mathcal{A}}_{\beta_{M}}$ express the contributions to $\breve{\gamma}_{M}^{E(1)}$ from the transitions at $z=z_{L}$ and $z=z_{R}$ and the terms proportional to $\check{\mathcal{B}}_{\beta_{M}}$ express the contributions coming from the region $z_{L}<z<z_{R}$ where the curvature is the constant $\check{\kappa}_{0}$.

The only non-zero contribution to $\check{\mathcal{A}}_{\sigma}(t, z)$ arises from the region $-c t<z \leq c t$. The term involving $\kappa_{0}^{\prime}\left(c t^{\prime}\right)$ in the integrand now follows from the relations

$$
\frac{\partial}{\partial z}\left(\Theta\left(z-z_{L}\right)-\Theta\left(z-z_{R}\right)\right)=\delta\left(z-z_{L}\right)-\delta\left(z-z_{R}\right),
$$

and $\delta\left(z / z_{0}\right)=\left|z_{0}\right| \delta(z)$ yielding

$$
\check{\mathcal{A}}_{\sigma}(t, z)=\frac{\check{\kappa}_{0}}{2 c^{2}} \int_{0}^{t_{c}^{\prime}(t, z)} d t^{\prime}\left(\delta\left(t^{\prime}-\frac{z_{L}}{c}\right)-\delta\left(t^{\prime}-\frac{z_{R}}{c}\right)\right) J_{0}\left(\sigma s_{c}\left(t, z \mid t^{\prime}\right)\right) .
$$

Hence, see Fig 3 ,

- For $(-c t<z<c t) \cap\left(z_{R}<c t_{c}^{\prime}(t, z)\right)$, (Region $\left.R_{1}\right)$

$$
\check{\mathcal{A}}_{\sigma}(t, z)=\frac{\check{\kappa}_{0}}{2 c^{2}}\left\{J_{0}\left(\sigma s_{c}\left(t, z \mid \frac{z_{L}}{c}\right)\right)-J_{0}\left(\sigma s_{c}\left(t, z \mid \frac{z_{R}}{c}\right)\right)\right\},
$$

- for $(-c t<z<c t) \cap\left(z_{R} \geq c t_{c}^{\prime}(t, z)\right) \cap\left(z_{L}<c t_{c}^{\prime}(t, z)\right)$, (Region $\left.R_{2}\right)$

$$
\check{\mathcal{A}}_{\sigma}(t, z)=\frac{\check{\kappa}_{0}}{2 c^{2}} J_{0}\left(\sigma s_{c}\left(t, z \mid \frac{z_{L}}{c}\right)\right),
$$

- for $(-c t<z<c t) \cap\left(z_{L} \geq c t_{c}^{\prime}(t, z)\right)$, (Region $\left.R_{3}\right)$

$$
\check{\mathcal{A}}_{\sigma}(t, z)=0 .
$$




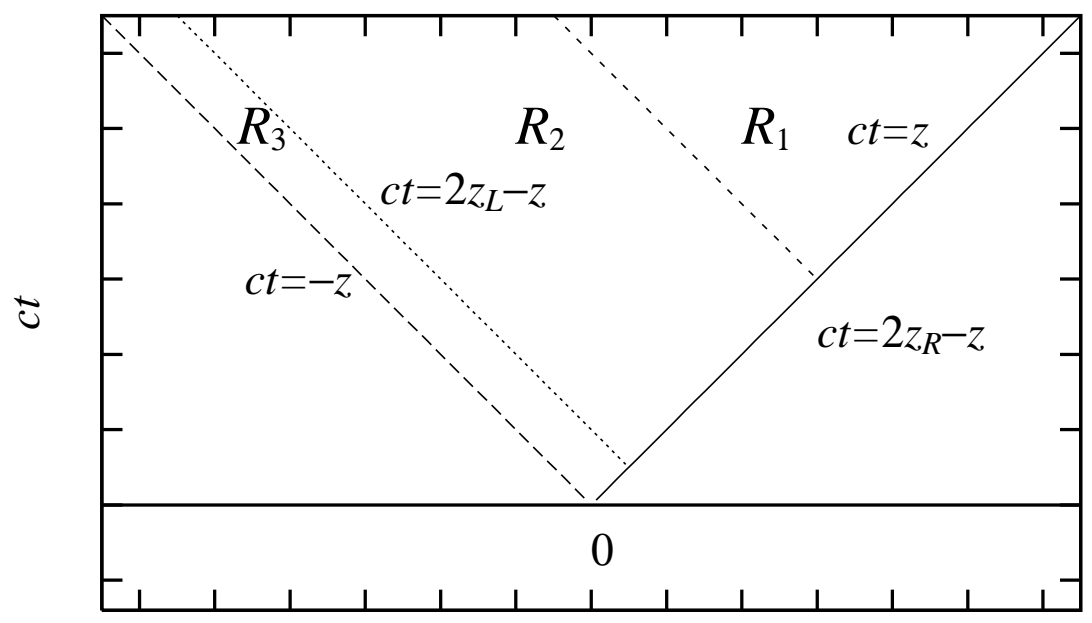

$z$

FIG. 3: Integration ranges for $\check{A}_{\sigma}(t, z)$ and $\check{B}_{\sigma}(t, z) . R_{1}$ is the triangle in the upper right-hand corner i.e., $(-c t<z<c t) \cap\left(z_{R}<\right.$ $\left.c t_{c}^{\prime}(t, z)\right) . R_{2}$ is the adjacent large rectangle: $(-c t<z<c t) \cap\left(z_{R} \geq c t_{c}^{\prime}(t, z)\right) \cap\left(z_{L}<c t_{c}^{\prime}(t, z)\right)$, and $R_{3}$ is the adjacent smaller rectangle: $(-c t<z<c t) \cap\left(z_{L} \geq c t_{c}^{\prime}(t, z)\right)$.

Since at $z_{R}=c t_{c}^{\prime}$ and $z_{L}=c t_{c}^{\prime}$,

$$
s_{c}\left(t, 2 z_{R}-c t \mid \frac{z_{R}}{c}\right)=0, \quad \text { and } \quad s_{c}\left(t, 2 z_{L}-c t \mid \frac{z_{L}}{c}\right)=0 .
$$

A simple calculation from(134), shows that with $z=c t$,

- for $z_{R}<z$,

$$
\check{\mathcal{A}}_{\sigma}(t, z)=0,
$$

- for $z_{R}=z$,

$$
\check{\mathcal{A}}_{\sigma}(t, z)=\frac{\check{\kappa}_{0}}{4 c^{2}}
$$

- for $z_{L}<z<z_{R}$,

$$
\check{\mathcal{A}}_{\sigma}(t, z)=\frac{\check{\kappa}_{0}}{2 c^{2}}
$$

- for $z=z_{L}$,

$$
\check{\mathcal{A}}_{\sigma}(t, z)=\frac{\check{\kappa}_{0}}{4 c^{2}}
$$

- for $z<z_{L}$,

$$
\check{\mathcal{A}}_{\sigma}(t, z)=0 \text {. }
$$

Similarly since

$$
\check{\mathcal{B}}_{\sigma}(t, z)=0,
$$

for $-c t<z<c t$, and $\left(z<z_{L}\right) \cup\left(z \geq z_{R}\right)$, we must evaluate

$$
\check{\mathcal{B}}_{\sigma}(t, z)=\frac{\sigma \check{\kappa}_{0}}{2 c} \int_{0}^{t_{c}^{\prime}(t, z)} d t^{\prime} \frac{z-c t^{\prime}}{s_{c}\left(t, z \mid t^{\prime}\right)} J_{1}\left(\sigma s_{c}\left(t, z \mid t^{\prime}\right)\right),
$$


with $-c t<z<c t$ and $z_{L} \leq z<z_{R}$ where $\kappa_{0}(z) \neq 0$.

Write the integral in $\check{\mathcal{B}}_{\sigma}$ as

$$
\Lambda_{\sigma}(t, z):=\int_{0}^{t_{c}^{\prime}(t, z)} d t^{\prime} \frac{z-c t^{\prime}}{s_{c}\left(t, z \mid t^{\prime}\right)} J_{1}\left(\sigma s_{c}\left(t, z \mid t^{\prime}\right)\right),
$$

and change variables, $t^{\prime} \mapsto s_{c}^{\prime}\left(t^{\prime}\right)$ with fixed $t$ and $z$,

$$
s_{c}^{\prime}\left(t^{\prime}\right):=\sqrt{c^{2}\left(t-t^{\prime}\right)^{2}-\left(z-c t^{\prime}\right)^{2}} .
$$

Then with

$$
d t^{\prime}=d s_{c}^{\prime} \frac{s_{c}^{\prime}}{c(z-c t)}, \quad z-c t^{\prime}=\frac{(z-c t)^{2}-s_{c}^{\prime 2}}{2(z-c t)},
$$

Eq.(143) can be expressed

$$
\Lambda_{\sigma}=\int_{\sqrt{(c t)^{2}-z^{2}}}^{0} d s_{c}^{\prime} \frac{(z-c t)^{2}-s_{c}^{\prime 2}}{2 c(z-c t)^{2}} J_{1}\left(\sigma s_{c}^{\prime}\right)
$$

since

$$
s_{c}(t, z \mid 0)=\sqrt{(c t)^{2}-z^{2}}, \quad s_{c}\left(t, z \mid t_{c}^{\prime}(t, z)\right)=0 .
$$

This integral can now be evaluated using

$$
\int d z J_{1}(\sigma z)=-\frac{1}{\sigma} J_{0}(\sigma z), \quad \int d z z^{2} J_{1}(\sigma z)=\frac{z^{2}}{\sigma} J_{2}(\sigma z) .
$$

Hence

$$
\begin{aligned}
\Lambda_{\sigma} & =\frac{1}{2 c} \int_{\sqrt{(c t)^{2}-z^{2}}}^{0} d s_{c}^{\prime} J_{1}\left(\sigma s_{c}^{\prime}\right)-\frac{1}{2 c(z-c t)^{2}} \int_{\sqrt{(c t)^{2}-z^{2}}}^{0} d s_{c}^{\prime} s_{c}^{\prime 2} J_{1}\left(\sigma s_{c}^{\prime}\right) \\
& =\frac{1}{2 c} \frac{(-1)}{\sigma}\left[J_{0}\left(\sigma s_{c}^{\prime}\right)\right]_{\sqrt{(c t)^{2}-z^{2}}}^{0}-\frac{1}{2 c(z-c t)^{2}} \frac{1}{\sigma}\left[s_{c}^{\prime 2} J_{2}\left(\sigma s_{c}^{\prime}\right)\right]_{\sqrt{(c t)^{2}-z^{2}}}^{0}
\end{aligned}
$$

or with $J_{0}(0)=1$ and $J_{2}(0)=0$,

$$
\Lambda_{\sigma}(t, z)=\frac{1}{2 c \sigma}\left(-1+J_{0}\left(\sigma \sqrt{(c t)^{2}-z^{2}}\right)\right)+\frac{(c t)^{2}-z^{2}}{2 c(z-c t)^{2} \sigma} J_{2}\left(\sigma \sqrt{(c t)^{2}-z^{2}}\right)
$$

valid in the regime $-c t<z<c t$.

Finally for $z=c t$,

$$
\Lambda_{\sigma}=0 .
$$

Thus the function $\breve{\gamma}_{M}^{E(1)}(t, z)$ is given in the indicated domains (See Fig 3 ) by:

- for $(z \leq-c t) \cup(z \geq c t)$, (Outside the regions $R_{1}, R_{2}$ and $R_{3}$ )

$$
\breve{\gamma}_{M}^{E(1)}(t, z)=0,
$$

- for $(-c t<z<c t) \cap\left(z_{L} \geq c t_{c}^{\prime}(t, z)\right)$, (Region $\left.R_{3}\right)$

$$
\breve{\gamma}_{M}^{E(1)}(t, z)=\left(l_{M}^{\left(r_{0}, \theta_{0}\right)}-p_{M}^{\left(r_{0}, \theta_{0}\right)}\right) \frac{\beta_{M} \check{\kappa}_{0}}{2 c} \Lambda_{\beta_{M}}(t, z),
$$

- for $(-c t<z<c t) \cap\left(z_{L}<c t_{c}^{\prime}(t, z)\right) \cap\left(z_{R} \geq c t_{c}^{\prime}(t, z)\right)$, (Region $\left.R_{2}\right)$

$$
\begin{aligned}
\breve{\gamma}_{M}^{E(1)}(t, z)= & \left(-l_{M}^{\left(r_{0}, \theta_{0}\right)}+p_{M}^{\left(r_{0}, \theta_{0}\right)}+s_{M}^{\left(r_{0}, \theta_{0}\right)}\right) \frac{\check{\kappa}_{0}}{2 c^{2}} J_{0}\left(\beta_{M} s_{c}\left(t, z \mid \frac{z_{L}}{c}\right)\right) \\
& +\left(l_{M}^{\left(r_{0}, \theta_{0}\right)}-p_{M}^{\left(r_{0}, \theta_{0}\right)}\right) \frac{\beta_{M} \check{\kappa}_{0}}{2 c} \Lambda_{\beta_{M}}(t, z),
\end{aligned}
$$


- for $(-c t<z<c t) \cap\left(z_{R}<c t_{c}^{\prime}(t, z)\right)$, (Region $\left.R_{1}\right)$

$$
\begin{aligned}
& \breve{\gamma}_{M}^{E(1)}(t, z)=\left(-l_{M}^{\left(r_{0}, \theta_{0}\right)}+p_{M}^{\left(r_{0}, \theta_{0}\right)}+s_{M}^{\left(r_{0}, \theta_{0}\right)}\right) \frac{\check{\kappa}_{0}}{2 c^{2}} \\
& \times\left\{J_{0}\left(\beta_{M} s_{c}\left(t, z \mid \frac{z_{L}}{c}\right)\right)-J_{0}\left(\beta_{M} s_{c}\left(t, z \mid \frac{z_{R}}{c}\right)\right)\right\} \\
&+\left(l_{M}^{\left(r_{0}, \theta_{0}\right)}-p_{M}^{\left(r_{0}, \theta_{0}\right)}\right) \frac{\beta_{M} \check{\kappa}_{0}}{2 c} \Lambda_{\beta_{M}}(t, z)
\end{aligned}
$$

The explicit longitudinal wake potential in this case now follows from (128) and (138):

$$
\mathcal{W}_{\|}^{\left(r_{0}, \theta_{0}\right)}(\epsilon, \widetilde{s})=\mathcal{W}_{\| M, \text { edges }}^{\left(r_{0}, \theta_{0}\right)}(\epsilon, \widetilde{s})+\mathcal{W}_{\| M, \tilde{\kappa}_{0}}^{\left(r_{0}, \theta_{0}\right)}(\epsilon, \widetilde{s})+\mathcal{O}\left(\epsilon^{2}\right)
$$

Here, $\mathcal{W}_{\| M, \text { edges }}^{\left(r_{0}, \theta_{0}\right)}$ expresses the contributions from the abrupt transitions in curvature at $z=z_{L}$ and $z=z_{R}$, while $\mathcal{W}_{\| M, \check{\kappa}_{0}}^{\left(r_{0}, \theta_{0}\right)}$ denotes that from the region of constant curvature $z_{L}<z<z_{R}$. Splitting the range of integration in (128) according the domains associated with $\breve{\gamma}_{M}^{E(1)}$ (See Fig 5 ) one has

$$
\begin{array}{r} 
\\
\mathcal{W}_{\| M, \text { edges }}^{\left(r_{0}, \theta_{0}\right)}(\epsilon, \widetilde{s})=\epsilon \frac{\mathcal{N}_{M}^{2}}{Q_{\text {tot }}}\left(l_{M}^{\left(r_{0}, \theta_{0}\right)}-p_{M}^{\left(r_{0}, \theta_{0}\right)}-s_{M}^{\left(r_{0}, \theta_{0}\right)}\right) \\
\times\left(\int_{-\widetilde{s} / 2}^{z_{L}-\widetilde{s} / 2}+\int_{z_{L}-\widetilde{s} / 2}^{z_{R}+\widetilde{s} / 2}+\int_{z_{R}+\widetilde{s} / 2}^{\infty}\right) d z \check{\mathcal{A}}_{\beta_{M}}\left(\frac{z+\widetilde{s}}{c}, z\right)+\mathcal{O}\left(\epsilon^{2}\right),
\end{array}
$$

and

$$
\overline{\mathcal{W}_{\| M, \check{\kappa}_{0}}^{\left(r_{0}, \theta_{0}\right)}}(\epsilon, \widetilde{s})=-\epsilon \frac{\mathcal{N}_{M}^{2}}{Q_{t o t}}\left(l_{M}^{\left(r_{0}, \theta_{0}\right)}-p_{M}^{\left(r_{0}, \theta_{0}\right)}\right) \int_{z_{L}}^{z_{R}} d z \check{\mathcal{B}}_{\beta_{M}}\left(\frac{z+\widetilde{s}}{c}, z\right)+\mathcal{O}\left(\epsilon^{2}\right) .
$$

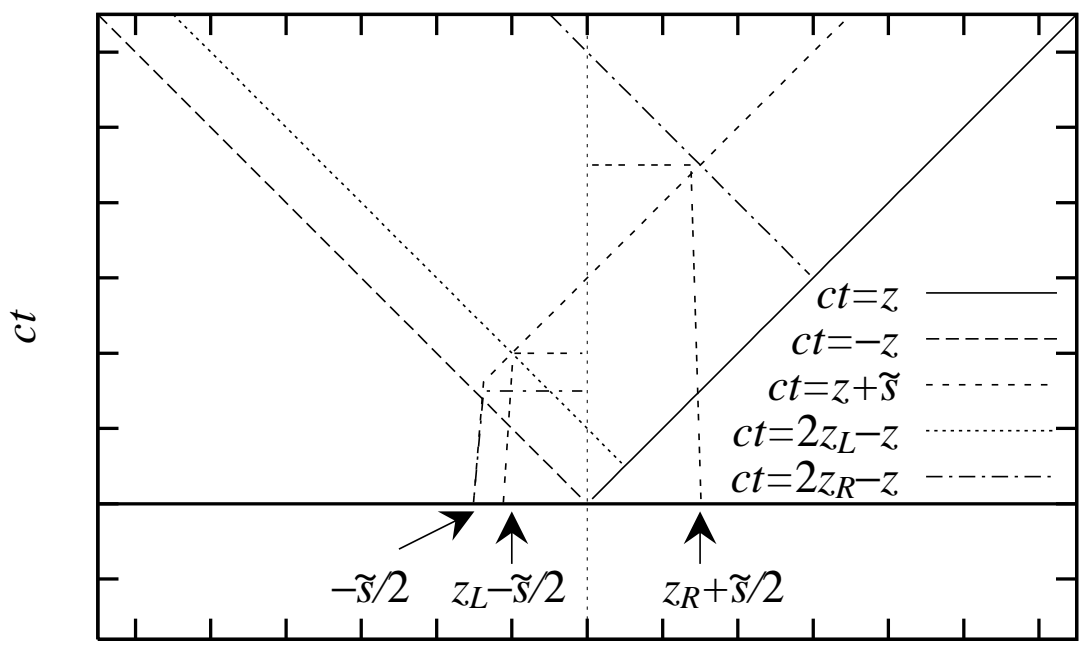

$z$

FIG. 4: Integration ranges for $\overline{\mathcal{W}_{\| M, \text { edges }}^{\left(r_{0}, \theta_{0}\right)}}$.

Again using the relation

$$
\int d z z^{\nu / 2} J_{\nu}(\sqrt{\sigma z})=\frac{2}{\sqrt{\sigma}} z^{(1+\nu) / 2} J_{\nu+1}(\sqrt{\sigma z})
$$


with $\nu=0$ the integrals in (148) are evaluated as

$$
\begin{aligned}
& \left(\int_{-\widetilde{s} / 2}^{z_{L}-\widetilde{s} / 2}+\int_{z_{L}-\widetilde{s} / 2}^{z_{R}+\widetilde{s} / 2}+\int_{z_{R}+\widetilde{s} / 2}^{\infty}\right) d z \check{\mathcal{A}}_{\beta_{M}}\left(\frac{z+\widetilde{s}}{c}, z\right) \\
& =0+\frac{\check{\kappa}_{0}}{2 c^{2}} \int_{z_{L}-\widetilde{s} / 2}^{z_{R}+\widetilde{s} / 2} d z J_{0}\left(\beta_{M} s_{c}\left(\frac{z+\widetilde{s}}{c}, z \mid \frac{z_{L}}{c}\right)\right) \\
& +\frac{\check{\kappa}_{0}}{2 c^{2}} \int_{z_{R}+\widetilde{s} / 2}^{\infty} d z\left\{J_{0}\left(\beta_{M} s_{c}\left(\frac{z+\widetilde{s}}{c}, z \mid \frac{z_{L}}{c}\right)\right)-J_{0}\left(\beta_{M} s_{c}\left(\frac{z+\widetilde{s}}{c}, z \mid \frac{z_{R}}{c}\right)\right)\right\} \\
& =\frac{\check{\kappa}_{0}}{2 c^{2}} \int_{0}^{\widetilde{s}} d z^{\prime} J_{0}\left(\beta_{M} \sqrt{2 \widetilde{s} z^{\prime}}\right)=\frac{\check{\kappa}_{0}}{\sqrt{2} c^{2} \beta_{M}} J_{1}\left(\sqrt{2} \beta_{M} \widetilde{s}\right),
\end{aligned}
$$

independent of $z_{L}$ and $z_{R}$. The integral involving $\check{\mathcal{B}}_{\sigma}$ in (149) can be similarly evaluated using (150) with $\nu=2$

$$
\begin{gathered}
\int_{z_{L}}^{z_{R}} d z \check{\mathcal{B}}_{\beta_{M}}\left(\frac{z+\widetilde{s}}{c}, z\right)=\frac{\beta_{M} \check{\kappa}_{0}}{2 c} \int_{z_{L}}^{z_{R}} d z \Lambda_{\beta_{M}}\left(\frac{z+\widetilde{s}}{c}, z\right) \\
=\frac{\beta_{M} \check{\kappa}_{0}}{2 c} \int_{z_{L}}^{z_{R}} d z\left\{\frac{1}{2 c \beta_{M}}\left(-1+J_{0}\left(\beta_{M} \sqrt{2 \widetilde{s} z+\widetilde{s}^{2}}\right)\right)\right. \\
\left.\quad+\frac{2 z \widetilde{s}+\widetilde{s}^{2}}{2 c \beta_{M} \widetilde{s}^{2}} J_{2}\left(\beta_{M} \sqrt{2 \widetilde{s} z+\widetilde{s}^{2}}\right)\right\} \\
=\frac{\check{\kappa}_{0}}{4 c^{2}}\left[-z^{\prime}+\frac{\sqrt{2}}{\beta_{M} \sqrt{\widetilde{s}}}\left\{\sqrt{z^{\prime}} J_{1}\left(\beta_{M} \sqrt{2 \widetilde{s} z^{\prime}}\right)\right.\right. \\
\left.\left.+\frac{2 z^{\prime 3 / 2}}{\widetilde{s}} J_{3}\left(\beta_{M} \sqrt{2 \widetilde{s} z^{\prime}}\right)\right\}\right]_{z^{\prime}=z_{L}+\widetilde{s} / 2}^{z^{\prime}=z_{R}+\widetilde{s} / 2} .
\end{gathered}
$$

Finally from (148), (151) one has

$$
\overline{\mathcal{W}_{\| M, \text { edges }}^{\left(r_{0}, \theta_{0}\right)}}(\epsilon, \widetilde{s})=\epsilon \frac{\check{\kappa}_{0}}{\sqrt{2} \beta_{M}}\left(\breve{l}_{M}^{\left(r_{0}, \theta_{0}\right)}-\breve{p}_{M}^{\left(r_{0}, \theta_{0}\right)}-\breve{s}_{M}^{\left(r_{0}, \theta_{0}\right)}\right) J_{1}\left(\sqrt{2} \beta_{M} \widetilde{s}\right)+\mathcal{O}\left(\epsilon^{2}\right),
$$

and from (149), 152)

$$
\begin{aligned}
& \overline{\mathcal{W}_{\| M, \check{\kappa}_{0}}^{\left(r_{0}, \theta_{0}\right)}}(\epsilon, \widetilde{s})=-\epsilon \frac{\check{\kappa}_{0}}{4}\left(\breve{l}_{M}^{\left(r_{0}, \theta_{0}\right)}-\breve{p}_{M}^{\left(r_{0}, \theta_{0}\right)}\right)\left[-\left(z_{R}-z_{L}\right)+\frac{\sqrt{2}}{\beta_{M} \sqrt{\widetilde{s}}}\right. \\
& \times\left\{\sqrt{z_{R}+\frac{\widetilde{s}}{2}} J_{1}\left(\beta_{M} \sqrt{2 \widetilde{s}\left(z_{R}+\frac{\widetilde{s}}{2}\right)}\right)\right. \\
& +\frac{2\left(z_{R}+\frac{\widetilde{s}}{2}\right)^{3 / 2}}{\widetilde{s}} J_{3}\left(\beta_{M} \sqrt{2 \widetilde{s}\left(z_{R}+\frac{\widetilde{s}}{2}\right)}\right) \\
& -\sqrt{z_{L}+\frac{\widetilde{s}}{2}} J_{1}\left(\beta_{M} \sqrt{2 \widetilde{s}\left(z_{L}+\frac{\widetilde{s}}{2}\right)}\right) \\
& \left.\left.-\frac{2\left(z_{L}+\frac{\widetilde{s}}{2}\right)^{3 / 2}}{\widetilde{s}} J_{3}\left(\beta_{M} \sqrt{2 \widetilde{s}\left(z_{L}+\frac{\widetilde{s}}{2}\right)}\right)\right\}\right]+\mathcal{O}\left(\epsilon^{2}\right),
\end{aligned}
$$


where, from (139), (140), (141) we introduce the abbreviations

$$
\begin{aligned}
\breve{l}_{M}^{\left(r_{0}, \theta_{0}\right)}:= & \frac{\mathcal{N}_{M}^{2}}{c^{2} Q_{t o t}} l_{M}^{\left(r_{0}, \theta_{0}\right)}=2 \mu c^{2} \sum_{N}^{\prime} \frac{1}{\mathcal{N}_{N}^{2}}\left(-E_{M, N}^{\Phi}+\frac{C_{M, N}^{\Phi}-D_{M, N}^{\Phi}}{\beta_{N}^{2}}\right) \\
& \times J_{n}\left(x_{q(n)} \frac{r_{0}}{a}\right) e^{-i n \theta_{0}}, \\
\breve{p}_{M}^{\left(r_{0}, \theta_{0}\right)}:= & \frac{\mathcal{N}_{M}^{2}}{c^{2} Q_{t o t}} p_{M}^{\left(r_{0}, \theta_{0}\right)}=\mu c^{2} r_{0} \cos \theta_{0} J_{m}\left(x_{p(m)} \frac{r_{0}}{a}\right) e^{-i m \theta_{0}}, \\
\breve{s}_{M}^{\left(r_{0}, \theta_{0}\right)}:= & \frac{\mathcal{N}_{M}^{2}}{c^{2} Q_{t o t}} s_{M}^{\left(r_{0}, \theta_{0}\right)}=\mu c^{2} \sum_{N}^{\prime} \frac{1}{\mathcal{N}_{N}^{2}}\left(-E_{M, N}^{\Phi}+\frac{C_{M, N}^{\Phi}-D_{M, N}^{\Phi}}{\beta_{N}^{2}}\right) \\
& \times J_{n}\left(x_{q(n)} \frac{r_{0}}{a}\right) e^{-i n \theta_{0}} .
\end{aligned}
$$

It is worth noting that the expressions for the wake potentials are independent of $Q_{t o t}$.

With the following dimensionless variables for some length $L$

$$
\widehat{\kappa}_{0}:=L \check{\kappa}_{0}, \quad \widehat{s}:=\frac{\widetilde{s}}{L}, \quad \widehat{\beta}_{M}:=L \beta_{M}, \quad \widehat{z}_{R}:=\frac{z_{R}}{L}, \quad \widehat{z}_{L}:=\frac{z_{L}}{L},
$$

one may introduce the dimensionless quantities

$$
\begin{aligned}
& \zeta_{M, 1}(\widehat{s}):=\frac{\widehat{\kappa}_{0}}{\widehat{\beta}_{M}} J_{1}\left(\sqrt{2} \widehat{\beta}_{M} \widehat{s}\right), \\
& \zeta_{M, 2}(\widehat{s}):=\widehat{\kappa}_{0}\left[\left(\widehat{z}_{R}-\widehat{z}_{L}\right)-\frac{\sqrt{2}}{\widehat{\beta}_{M} \sqrt{\widehat{s}}}\right. \\
& \times\left\{\sqrt{\widehat{z}_{R}+\frac{\widehat{s}}{2}} J_{1}\left(\beta_{M} \sqrt{2 \widehat{s}\left(\widehat{z}_{R}+\frac{\widehat{s}}{2}\right)}\right)\right. \\
& +\frac{2\left(\widehat{z}_{R}+\frac{\widehat{s}}{2}\right)^{3 / 2}}{\widehat{s}} J_{3}\left(\widehat{\beta}_{M} \sqrt{2 \widehat{s}\left(\widehat{z}_{R}+\frac{\widehat{s}}{2}\right)}\right) \\
& -\sqrt{\widehat{z}_{L}+\frac{\widehat{s}}{2}} J_{1}\left(\widehat{\beta}_{M} \sqrt{2 \widehat{s}\left(\widehat{z}_{L}+\frac{\widehat{s}}{2}\right)}\right) \\
& \left.\left.-\frac{2\left(\widehat{z}_{L}+\frac{\widehat{s}}{2}\right)^{3 / 2}}{\widehat{s}} J_{3}\left(\widehat{\beta}_{M} \sqrt{2 \widehat{s}\left(\widehat{z}_{L}+\frac{\widehat{s}}{2}\right)}\right)\right\}\right],
\end{aligned}
$$

in terms of which

$$
\begin{aligned}
\overline{\mathcal{W}_{\| M, \text { edges }}^{\left(r_{0}, \theta_{0}\right)}}(\epsilon, \widetilde{s}) & =\frac{\epsilon}{\sqrt{2}} \zeta_{M, 1}(\widehat{s})\left(\breve{l}_{M}^{\left(r_{0}, \theta_{0}\right)}-\breve{p}_{M}^{\left(r_{0}, \theta_{0}\right)}-\breve{s}_{M}^{\left(r_{0}, \theta_{0}\right)}\right), \\
\overline{\mathcal{W}_{\| M, \breve{\kappa}_{0}}^{\left(r_{0}, \theta_{0}\right)}}(\epsilon, \widetilde{s}) & =\frac{\epsilon}{4} \zeta_{M, 2}(\widehat{s})\left(\breve{l}_{M}^{\left(r_{0}, \theta_{0}\right)}-\breve{p}_{M}^{\left(r_{0}, \theta_{0}\right)}\right) .
\end{aligned}
$$

Natural choices for $L$ include $L=a$ or $L=z_{R}-z_{L}$. In Fig 5 we plot $\zeta_{M, 1}$ and $\zeta_{M, 2}$ for the choice

$$
\widehat{\kappa}_{0}=1, \quad \widehat{\beta}_{M}=1, \quad \widehat{z}_{R}=2, \quad \widehat{z}_{L}=1 .
$$

In the regime $\widehat{s} \gg 1, \zeta_{M, 2}$ tends to $\widehat{\kappa}_{0}\left(\widehat{z}_{R}-\widehat{z}_{L}\right)$.

\section{SMOOTH CONVECTED LOCALIZED BUNCHES WITH FIXED TOTAL CHARGE}

In the previous section attention was concentrated on the ultrarelativistic limit. In this section, by contrast with §VI, we construct a moving source model with finite total charge $Q_{\text {tot }}$, and a smooth charge density satisfying (101) moving at less than the speed of light. Large numbers of charged particles moving with a common axial velocity may be modelled by a localised smooth distribution of electric charge with a prescribed convective axial velocity field with 


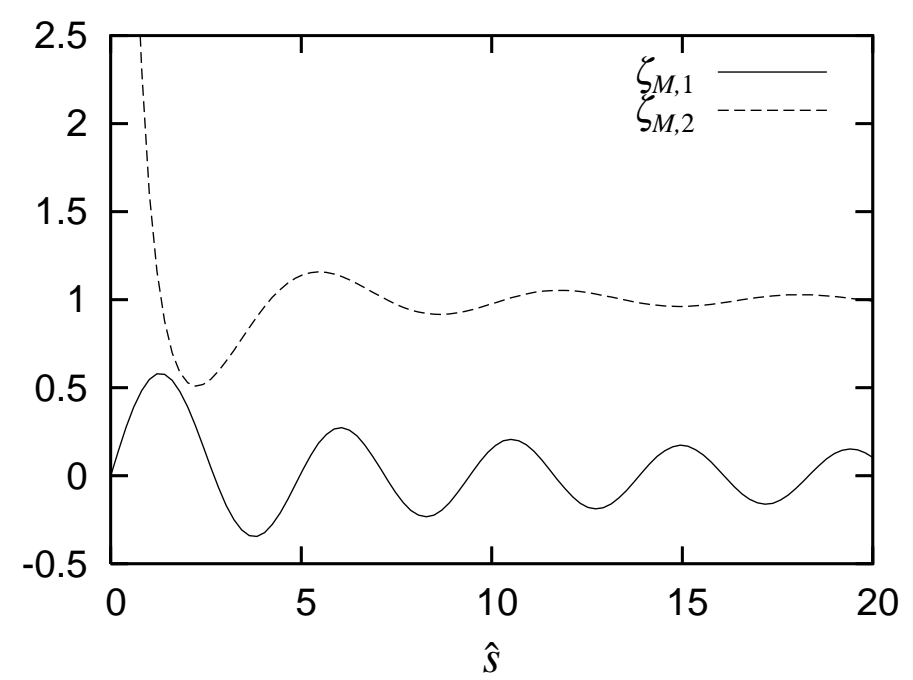

FIG. 5: Dimensionless profiles for contributions to $\mathcal{W}_{\|}^{\left(r_{0}, \theta_{0}\right)}(\widetilde{s})$ to $\mathcal{O}\left(\epsilon^{2}\right)$.

constant longitudinal speed $v<c_{0}$ (independent of the local curvature $\kappa(z)$ ), charge density $\rho(z-v t, r, \theta)$ and current density components $J_{r}=J_{\theta}=0, J_{0}(z-v t, r, \theta)=v \rho(z-v t, r, \theta)$. The localized charge density profile is thereby maintained as a function of the arc-length parameter $z$ as it travels along the beam pipe. Note that in this source model $J_{0}^{(0)}=J_{0}$ and all higher orders are taken zero.

We assume here that $\rho$ can be expressed as

$$
\rho\left(\epsilon, t, z, x_{1}, x_{2}\right)=Q(\epsilon, t) \rho^{\perp}\left(x_{1}, x_{2}\right) \rho^{\|}(z-v t),
$$

where $v$ is given $(v \leq c), \rho^{\perp}\left(x_{1}, x_{2}\right)$ and $\rho^{\|}(z-v t)$ are arbitrary smooth functions subject to

$$
\int_{\mathcal{D}} \rho^{\perp}\left(x_{1}, x_{2}\right) \hat{\#} 1=1, \quad \int_{-\infty}^{\infty} d z \rho^{\|}(z-v t)=1,
$$

and

$$
Q(\epsilon, t):=\frac{Q_{t o t}}{1-\epsilon\left\langle\rho^{\|} \kappa_{0}\right\rangle_{z}(t)\left\langle x_{1} \rho^{\perp}\right\rangle_{\mathcal{D}}}=Q_{t o t}+\epsilon Q_{t o t}\left\langle\rho^{\|} \kappa_{0}\right\rangle_{z}(t)\left\langle x_{1} \rho^{\perp}\right\rangle_{\mathcal{D}}+\mathcal{O}\left(\epsilon^{2}\right),
$$

with

$$
\begin{aligned}
\left\langle\rho^{\|} \kappa_{0}\right\rangle_{z}(t) & :=\int_{-\infty}^{\infty} d z \rho^{\|}(z-v t) \kappa_{0}(z), \\
\left\langle x_{1} \rho^{\perp}\right\rangle_{\mathcal{D}} & :=\int_{\mathcal{D}} x_{1} \rho^{\perp}\left(x_{1}, x_{2}\right) \hat{\#} 1 .
\end{aligned}
$$

Correspondingly, we define

$$
\begin{aligned}
\rho^{(0)}\left(z-v t, x_{1}, x_{2}\right) & :=Q_{t o t} \rho^{\perp}\left(x_{1}, x_{2}\right) \rho^{\|}(z-v t), \\
\rho^{(1)}\left(t, z, x_{1}, x_{2}\right) & :=Q_{t o t}\left\langle\rho^{\|} \kappa_{0}\right\rangle_{z}(t)\left\langle x_{1} \rho^{\perp}\right\rangle_{\mathcal{D}} \rho^{\perp}\left(x_{1}, x_{2}\right) \rho^{\|}(z-v t) .
\end{aligned}
$$

The currents $J_{0}^{(0)}$ and $J_{0}^{(1)}$ are defined by

$$
\begin{aligned}
J_{0}^{(0)}(z-v t, r, \theta) & :=v \rho^{(0)}\left(z-v t, x_{1}, x_{2}\right), \\
J_{0}^{(1)}(t, z, r, \theta) & :=v \rho^{(1)}\left(t, z, x_{1}, x_{2}\right) .
\end{aligned}
$$




\section{A. Lowest Order Fields from Moving Smooth Convected Localized Charge}

The equations for $\gamma_{N}^{H(0)}$ and $\gamma_{N}^{E(0)}$ are given by (85) and (86). The causal solutions are given by

$$
\begin{aligned}
\gamma_{N}^{H(0)}(t, z) & =\mathcal{H}_{\alpha_{N}}\left[\gamma_{N}^{H(0) i n i t}\right](t, z), \\
\gamma_{N}^{E(0)}(t, z) & =\mathcal{H}_{\beta_{N}}\left[\gamma_{N}^{E(0){ }^{(n i t}}\right](t, z)-\frac{\mu c^{2}}{\mathcal{N}_{N}^{2}}\left(c^{2}-v^{2}\right) \mathcal{I}_{\beta_{N}}\left[\overline{\rho_{N}^{(0) \prime}}\right](t, z),
\end{aligned}
$$

where $\rho_{N}^{(0)}$ is given by (187) and

$$
\rho_{N}^{(0) '}(t, z)=Q_{t o t} \rho^{\| \prime}(z-v t) \int_{\mathcal{D}} \rho^{\perp}\left(x_{1}, x_{2}\right) \Phi_{N} \hat{\#} 1
$$

In the ultra-relativistic limit, $v \rightarrow c$, the second term in $\gamma_{N}^{E(0)}(t, z)$ tends to zero.

\section{B. First Order Fields from Moving Convected Localized Charge}

The computation of the longitudinal fields follows along the lines detailed above for the point source. The distributional source is simply replaced by the smooth sources (153) at each respective order. For a general such source one obtains a system of fully coupled modal equations.

The causal solution to $\gamma_{M}^{H(1)}$ for $\gamma_{M}^{H(1)}$ can be written as

$$
\gamma_{M}^{H(1)}(t, z)=\mathcal{I}_{\alpha_{M}}\left[\widehat{K}_{M}^{H(1)}\right](t, z)+\mathcal{I}_{\alpha_{M}}\left[\widehat{L}_{M}^{H(1)}\right](t, z)
$$

As before the functional form of $\widehat{K}_{M}^{H(1)}(t, z)$ given in terms of $\gamma_{N}^{H(0)}$ and $\gamma_{N}^{E(0)}$ is the same as for $K_{M}^{H(1)}(t, z)$ defined in (114) but now, $\widehat{L}_{M}^{H(1)}(t, z)$ is calculated to be

$$
\widehat{L}_{M}^{H(1)}(t, z):=2 \frac{c^{2} v}{\mathcal{N}_{M}^{2}} \kappa_{0}(z) \sum_{N}^{\prime} \frac{G_{M, N}^{\bar{\Psi}, \Phi}}{\mathcal{N}_{N}^{2} \beta_{N}^{2}} \overline{\rho_{N}^{(0) \prime}}(t, z) .
$$

Similarly the causal solution for $\gamma_{M}^{E(1)}$ is written

$$
\begin{aligned}
\gamma_{M}^{E(1)}(t, z)= & \mathcal{I}_{\beta_{M}}\left[\widehat{K}_{M}^{E(1)}\right](t, z)+\mathcal{I}_{\beta_{M}}\left[\widehat{L}_{M}^{E(1)}\right](t, z)+\mathcal{I}_{\beta_{M}}\left[\widehat{P}_{M}^{E(1)}\right](t, z) \\
& +\mathcal{I}_{\beta_{M}}\left[\widehat{R}_{M}^{E(1)}\right](t, z)+\mathcal{I}_{\beta_{M}}\left[\widehat{S}_{M}^{E(1)}\right](t, z) .
\end{aligned}
$$

with the functional form of $\widehat{K}_{M}^{E(1)}(t, z)$ written in terms of $\gamma_{N}^{H(0)}$ and $\gamma_{N}^{E(0)}$ analogous to that of $K_{M}^{E(1)}(t, z)$ given in (115) and

$$
\begin{aligned}
& \widehat{L}_{M}^{E(1)}(t, z):=\frac{\mu c^{2}\left(c^{2}+v^{2}\right)}{\mathcal{N}_{M}^{2}} \kappa_{0}(z) \sum_{N}^{\prime} \frac{1}{\mathcal{N}_{N}^{2}} \\
& \times\left\{-E_{M, N}^{\Phi}+\frac{C_{M, N}^{\Phi}-D_{M, N}^{\Phi}}{\beta_{N}^{2}}\right\} \overline{\rho_{N}^{(0) \prime}}(t, z), \\
& \widehat{P}_{M}^{E(1)}(t, z):=-\frac{\mu c^{2} v}{\mathcal{N}_{M}^{2}} \overline{\dot{\rho}_{M}^{(1)}}(t, z), \\
& \widehat{R}_{M}^{E(1)}(t, z):=-\frac{\mu c^{4}}{\mathcal{N}_{M}^{2}} \int_{\mathcal{D}}\left\{\rho^{(1) \prime}-\left(\rho^{(0)} \kappa_{0}^{\prime}(z)+\rho^{(0) \prime} \kappa_{0}(z)\right) x_{1}\right\} \overline{\Phi_{M}} \hat{\#} 1, \\
& \widehat{S}_{M}^{E(1)}(t, z):=\frac{\mu c^{4}}{\mathcal{N}_{M}^{2}} \kappa_{0}^{\prime}(z) \sum_{N}^{\prime} \frac{1}{\mathcal{N}_{N}^{2}}\left(-E_{M, N}^{\Phi}+\frac{C_{M, N}^{\Phi}-D_{M, N}^{\Phi}}{\beta_{N}^{2}}\right) \overline{\rho_{N}^{(0)}}(t, z) .
\end{aligned}
$$

where

$$
\dot{\rho}_{M}^{(1)}=Q_{t o t}\left\langle x_{1} \rho^{\perp}\right\rangle_{\mathcal{D}} \frac{\partial}{\partial t}\left\{\left\langle\rho^{\|} \kappa_{0}\right\rangle_{z}(t) \rho^{\|}(z-v t)\right\} \int_{\mathcal{D}} \rho^{\perp}\left(x_{1}, x_{2}\right) \Phi_{M} \hat{\#} 1,
$$


and

$$
\begin{aligned}
& \int_{\mathcal{D}}\left\{\rho^{(1) \prime}-\left(\rho^{(0)} \kappa_{0}^{\prime}(z) \rho^{(0) \prime} \kappa_{0}(z)\right) x_{1}\right\} \Phi_{M} \hat{\#} 1 \\
& =Q_{t o t}\left\langle\rho^{\|} \kappa_{0}\right\rangle_{z}(t)\left\langle x_{1} \rho^{\perp}\right\rangle_{\mathcal{D}} \rho^{\| \prime}(z-v t) \int_{\mathcal{D}} \rho^{\perp}\left(x_{1}, x_{2}\right) \Phi_{M} \hat{\#} 1 \\
& \quad-Q_{t o t}\left\{\kappa_{0}^{\prime}(z) \rho^{\|}(z-v t)+\kappa_{0}(z) \rho^{\| \prime}(z-v t)\right\} \int_{\mathcal{D}} x_{1} \rho^{\perp}\left(x_{1}, x_{2}\right) \Phi_{M} \hat{\#} 1 .
\end{aligned}
$$

\section{Axially Symmetric Smooth Convected Localized Charge Distribution}

If the transverse distribution depends only on $r$, expressions for the electromagnetic 1-forms simplify. The source is axially symmetric if

$$
\rho^{\perp}\left(x_{1}, x_{2}\right)=\mathcal{R}(r)
$$

where $\mathcal{R}(r)$ is a smooth function satisfying

$$
\int_{0}^{a} d r r \mathcal{R}(r)=\frac{1}{2 \pi}
$$

It follows that

$$
\rho_{N}^{(0) \prime}(t, z)=2 \pi Q_{t o t} \delta_{n, 0} \rho^{\| \prime}(z-v t) \int_{0}^{a} d r r \mathcal{R}(r) J_{0}\left(x_{q(0)} \frac{r}{a}\right)
$$

The expressions for $\gamma_{M}^{H(1)}$ and $\gamma_{M}^{E(1)}$ simplify and explicit expressions for the overlap coefficients can be computed in terms of transverse projections of the radial profile $\mathcal{R}(r)$. We consider this case in simplifying the situation. From (157), it follows immediately that

$$
\begin{aligned}
\widehat{L}_{M}^{H(1)}(t, z)= & \left(\delta_{m, 1}+\delta_{m,-1}\right) \frac{4 \pi c^{2} v}{\mathcal{N}_{M}^{2}} Q_{t o t} \kappa_{0}(z) \rho^{\| \prime}(z-v t) \\
& \times \sum_{q(0) \in \mathbb{N}} \frac{G_{M, 0, q(0)}^{\bar{\Psi}, \Phi}}{\mathcal{N}_{0, q(0)}^{2} \beta_{0, q(0)}^{2}} \int_{0}^{a} d r r \mathcal{R}(r) J_{0}\left(x_{q(0)} \frac{r}{a}\right),
\end{aligned}
$$

and

$$
\begin{aligned}
& \widehat{L}_{M}^{E(1)}(t, z)=\left(\delta_{m, 1}+\delta_{m,-1}\right) \frac{2 \pi \mu c^{2}\left(c^{2}+v^{2}\right) Q_{t o t}}{\mathcal{N}_{M}^{2}} \kappa_{0}(z) \rho^{\| \prime}(z-v t) \\
& \times \sum_{q(0) \in \mathbb{N}} \frac{1}{\mathcal{N}_{0, q(0)}^{2}}\left\{-E_{M, 0, q(0)}^{\Phi}+\frac{C_{M, 0, q(0)}^{\Phi}-D_{M, 0, q(0)}^{\Phi}}{\beta_{0, q(0)}^{2}}\right\} \\
& \quad \times \int_{0}^{a} d r r \mathcal{R}(r) J_{0}\left(x_{q(0)} \frac{r}{a}\right),
\end{aligned}
$$

Then, since

$$
\left\langle x_{1} \rho^{\perp}\right\rangle_{\mathcal{D}}=0
$$

one has

$$
\overline{\dot{\rho}_{M}^{(1)}}(t, z)=0 .
$$

and therefore,

$$
\widehat{P}_{M}^{E(1)}(t, z)=0 .
$$


Finally, using (158) and

$$
\int_{\mathcal{D}} x_{1} \rho^{\perp} \overline{\Phi_{M}} \hat{\#} 1=\pi\left(\delta_{m,-1}+\delta_{m, 1}\right) \int_{0}^{a} d r r^{2} \mathcal{R}(r) J_{m}\left(x_{p(m)} \frac{r}{a}\right),
$$

one has

$$
\begin{aligned}
& \int_{\mathcal{D}}\left\{\rho^{(1) \prime}-\left(\rho^{(0)} \kappa_{0}^{\prime}(z)+\rho^{(0) \prime} \kappa_{0}(z)\right) x_{1}\right\} \overline{\Phi_{M}} \hat{\#} 1 \\
& =-Q_{t o t} \pi\left(\delta_{m,-1}+\delta_{m, 1}\right)\left(\kappa_{0}(z) \rho^{\| \prime}(z-v t)+\kappa_{0}^{\prime}(z) \rho^{\|}(z-v t)\right) \\
& \quad \times \int_{0}^{a} d r r^{2} \mathcal{R}(r) J_{m}\left(x_{p(m)} \frac{r}{a}\right) .
\end{aligned}
$$

It follows that

$$
\begin{aligned}
& \widehat{R}_{M}^{E(1)}(t, z)=\frac{\mu c^{4}}{\mathcal{N}_{M}^{2}} Q_{t o t} \pi\left(\delta_{m,-1}+\delta_{m, 1}\right) \\
& \times\left(\kappa_{0}(z) \rho^{\| \prime}(z-v t)+\kappa_{0}^{\prime}(z) \rho^{\|}(z-v t)\right) \int_{0}^{a} d r r^{2} \mathcal{R}(r) J_{m}\left(x_{p(m)} \frac{r}{a}\right) .
\end{aligned}
$$

and

$$
\begin{aligned}
& \widehat{S}_{M}^{E(1)}(t, z)=\left(\delta_{m, 1}+\delta_{m,-1}\right) \frac{2 \pi Q_{t o t}}{\mathcal{N}_{M}^{2}} \mu c^{4} \kappa_{0}^{\prime}(z) \\
& \quad \times \sum_{q(0) \in \mathbb{N}} \frac{1}{\mathcal{N}_{0, q(0)}^{2}}\left(-E_{M, 0, q(0)}^{\Phi}+\frac{C_{M, 0, q(0)}^{\Phi}-D_{M, 0, q(0)}^{\Phi}}{\beta_{0, q(0)}^{2}}\right) .
\end{aligned}
$$

In summary, for a smooth axially symmetric charge distribution, $\gamma_{M}^{H(1)}$ and $\gamma_{M}^{E(1)}$ can be written as

$$
\gamma_{M}^{H(1)}(t, z)=\mathcal{I}_{\alpha_{M}}\left[\widehat{K}_{M}^{H(1)}\right](t, z)+\left(\delta_{m,-1}+\delta_{m, 1}\right) \mathcal{I}_{\beta_{M}}\left[\widehat{L}_{M}^{H(1)}\right](t, z),
$$

and

$$
\begin{aligned}
\gamma_{M}^{E(1)}(t, z)= & \mathcal{I}_{\beta_{M}}\left[\widehat{K}_{M}^{E(1)}\right](t, z) \\
& +\left(\delta_{m,-1}+\delta_{m, 1}\right) \mathcal{I}_{\beta_{M}}\left[\widehat{L}_{M}^{E(1)}+\widehat{R}_{M}^{E(1)}+\widehat{S}_{M}^{E(1)}\right](t, z),
\end{aligned}
$$

using

$$
\left(\delta_{m,-1}+\delta_{m, 1}\right)^{2}=\left(\delta_{m,-1}+\delta_{m, 1}\right)
$$

\section{CONCLUSIONS}

This paper offers an analytic perturbative approach to the computation of electromagnetic fields generated by a variety of charged sources moving with prescribed motions in a perfectly conducting beam pipe of radius $a$ with planar curvature $\kappa(z)$. Results are given in terms of expressions involving powers of $|a \kappa(z)| \ll 1$ and $\left|a^{2} \kappa^{\prime}(z)\right|$. It has included a discussion of ultra-relativistic longitudinal wake potentials from which pipe impedances induced by $\kappa(z) \neq 0$ can be calculated. This has been explicitly illustrated for pipes with piecewise constant curvature modelling pipes with straight segments linked by circular arcs of (arbitrary) finite length.

There are a number of extensions that follow from this work. They include the effects of non-planarity and noncircular cross-sections, both varying with length along the pipe, resistive pipe boundary conditions, generalisations to dielectric channels and the computation of fields satisfying periodic boundary conditions in cyclic machines. Although more challenging the use of the geometric perturbation technique presented here is immediately applicable to these problems and will be discussed elsewhere. 


\section{Appendix A}

From the expressions for the orthonormal coframes (30) in adapted coordinates it is straightforward to derive the decompositions:

$$
\begin{aligned}
& \boldsymbol{d} \#\left(\boldsymbol{d} z \wedge \boldsymbol{d} \Psi_{M}\right)=-\alpha_{M}^{2}\left(1+\epsilon \kappa_{0}(z) r \cos \theta\right) \Psi_{M} \hat{\#} 1 \\
& -\epsilon \kappa_{0}(z)\left(\hat{\#} \boldsymbol{d} \Psi_{M}\right) \wedge(\cos \theta \boldsymbol{d} r-r \sin \theta \boldsymbol{d} \theta) \\
& -\epsilon \kappa_{0}^{\prime}(z) r \cos \theta\left(\hat{\#} \boldsymbol{d} \Psi_{M}\right) \wedge \boldsymbol{d} z+\mathcal{O}\left(\epsilon^{2}\right), \\
& \boldsymbol{d} \#\left(\boldsymbol{d} z \wedge \boldsymbol{d} \Phi_{N}\right)=-\beta_{N}^{2}\left(1+\epsilon \kappa_{0}(z) r \cos \theta\right) \Phi_{N} \hat{\#} 1 \\
& -\epsilon \kappa_{0}(z)\left(\hat{\#} \boldsymbol{d} \Phi_{N}\right) \wedge(\cos \theta \boldsymbol{d} r-r \sin \theta \boldsymbol{d} \theta) \\
& -\epsilon \kappa_{0}^{\prime}(z) r \cos \theta\left(\hat{\#} \boldsymbol{d} \Phi_{N}\right) \wedge \boldsymbol{d} z+\mathcal{O}\left(\epsilon^{2}\right), \\
& \boldsymbol{d} \# \boldsymbol{d} \Psi_{M}=-\left(1-\epsilon \kappa_{0}(z) r \cos \theta\right) \alpha_{M}^{2} \Psi_{M}(\hat{\#} 1) \wedge \boldsymbol{d} z \\
& -\epsilon \kappa_{0}(z)\left(\hat{\#} \boldsymbol{d} \Psi_{M}\right) \wedge(\cos \theta \boldsymbol{d} z \wedge \boldsymbol{d} r-r \sin \theta \boldsymbol{d} z \wedge \boldsymbol{d} \theta), \\
& \boldsymbol{d} \# \boldsymbol{d} \Phi_{N}=-\left(1-\epsilon \kappa_{0}(z) r \cos \theta\right) \beta_{N}^{2} \Phi_{N}(\hat{\#} 1) \wedge \boldsymbol{d} z \\
& -\epsilon \kappa_{0}(z)\left(\hat{\#} \boldsymbol{d} \Phi_{N}\right) \wedge(\cos \theta \boldsymbol{d} z \wedge \boldsymbol{d} r-r \sin \theta \boldsymbol{d} z \wedge \boldsymbol{d} \theta) \text {. }
\end{aligned}
$$

Furthermore if $\psi(r, \theta)$ represents either $\Phi_{N}(r, \theta)$ or $\Psi_{M}(r, \theta)$ one has

$$
\begin{gathered}
\hat{\#} \boldsymbol{d} \psi=\left(\partial_{r} \psi\right) r \boldsymbol{d} \theta-\frac{1}{r}\left(\partial_{\theta} \psi\right) \boldsymbol{d} r, \\
\# \boldsymbol{d} \psi=\left(1-\epsilon \kappa_{0}(z) r \cos \theta\right)(\hat{\#} \boldsymbol{d} \psi) \wedge \boldsymbol{d} z, \\
\#(\boldsymbol{d} z \wedge \boldsymbol{d} \psi)=\frac{\hat{\#} \boldsymbol{d} \psi}{1-\epsilon \kappa_{0}(z) r \cos \theta},
\end{gathered}
$$

and

$$
\# \boldsymbol{d} z=\frac{1}{1-\epsilon \kappa_{0}(z) r \cos \theta} \hat{\#} 1
$$

In terms of the symbol

$$
\Xi_{M, N}^{k}(\mathcal{F}, \mathcal{G}, x, y):=\int_{0}^{a} d r r^{k} \mathcal{F}_{m}\left(x_{p(m)} \frac{r}{a}\right) \mathcal{G}_{n}\left(y_{q(n)} \frac{r}{a}\right),
$$

the following overlap coefficients are defined:

$$
\begin{aligned}
C_{M, N}^{\Psi} & :=\int_{\mathcal{D}} \cos \theta \overline{\Psi_{M}} \frac{\partial \Psi_{N}}{\partial r} \hat{\#} 1 \\
& =\int_{0}^{2 \pi} d \theta \frac{e^{i \theta}+e^{-i \theta}}{2} e^{i(n-m) \theta} \int_{0}^{a} d r r J_{m}\left(x_{p(m)}^{\prime} \frac{r}{a}\right) \frac{x_{q(n)}^{\prime}}{a} J_{n}^{\prime}\left(x_{q(n)}^{\prime} \frac{r}{a}\right) \\
& =\pi\left(\delta_{n-m+1,0}+\delta_{n-m-1,0}\right) \frac{x_{q(n)}^{\prime}}{a} \int_{0}^{a} d r r J_{m}\left(x_{p(m)}^{\prime} \frac{r}{a}\right) J_{n}^{\prime}\left(x_{q(n)}^{\prime} \frac{r}{a}\right) \\
& =\pi\left(\delta_{n, m-1}+\delta_{n, m+1}\right) \frac{x_{q(n)}^{\prime}}{a} \Xi_{M, N}^{1}\left(J, J^{\prime}, x^{\prime}, x^{\prime}\right) .
\end{aligned}
$$




$$
\begin{aligned}
& D_{M, N}^{\Psi}:=\int_{\mathcal{D}} \frac{\sin \theta}{r} \overline{\Psi_{M}}\left(\partial_{\theta} \Psi_{N}\right) \hat{\#} 1=\pi n\left(\delta_{n, m-1}-\delta_{n, m+1}\right) \Xi_{M, N}^{0}\left(J, J, x^{\prime}, x^{\prime}\right), \\
& E_{M, N}^{\Psi}:=\int_{\mathcal{D}} r \cos \theta \overline{\Psi_{M}} \Psi_{N} \hat{\#} 1=\pi\left(\delta_{n, m-1}+\delta_{n, m+1}\right) \Xi_{M, N}^{2}\left(J, J, x^{\prime}, x^{\prime}\right), \\
& F_{M, N}^{\Psi}:=\int_{\mathcal{D}} r \cos \theta \boldsymbol{d} \overline{\Psi_{M}} \wedge \hat{\#} \boldsymbol{d} \Psi_{N}=\pi\left(\delta_{n, m-1}+\delta_{n, m+1}\right) \\
& \times\left(\frac{x_{p(m)}^{\prime}}{a} \frac{x_{q(n)}^{\prime}}{a} \Xi_{M, N}^{2}\left(J^{\prime}, J^{\prime}, x^{\prime}, x^{\prime}\right)+m n \Xi_{M, N}^{0}\left(J, J, x^{\prime}, x^{\prime}\right)\right), \\
& C_{M, N}^{\Phi}:=\int_{\mathcal{D}} \cos \theta \overline{\Phi_{M}}\left(\partial_{r} \Phi_{N}\right) \hat{\#} 1=\pi\left(\delta_{n, m-1}+\delta_{n, m+1}\right) \frac{x_{q(n)}}{a} \Xi_{M, N}^{1}\left(J, J^{\prime}, x, x\right), \\
& D_{M, N}^{\Phi}:=\int_{\mathcal{D}} \frac{\sin \theta}{r} \overline{\Phi_{M}}\left(\partial_{\theta} \Phi_{N}\right) \hat{\#} 1=\pi n\left(\delta_{n, m-1}-\delta_{n, m+1}\right) \Xi_{M, N}^{0}(J, J, x, x), \\
& E_{M, N}^{\Phi}:=\int_{\mathcal{D}} r \cos \theta \overline{\Phi_{M}} \Phi_{N} \hat{\#} 1=\pi\left(\delta_{n, m-1}+\delta_{n, m+1}\right) \Xi_{M, N}^{2}(J, J, x, x), \\
& F_{M, N}^{\Phi}:=\int_{\mathcal{D}} r \cos \theta \boldsymbol{d} \overline{\Phi_{M}} \wedge \hat{\#} \boldsymbol{d} \Phi_{N}=\pi\left(\delta_{n, m-1}+\delta_{n, m+1}\right) \\
& \times\left(\frac{x_{p(m)}}{a} \frac{x_{q(n)}}{a} \Xi_{M, N}^{2}\left(J^{\prime}, J^{\prime}, x, x\right)+m n \Xi_{M ; N}^{0}(J, J, x, x)\right) . \\
& G_{M, N}^{\bar{\Psi}, \Phi}:=\int_{\mathcal{D}} r \cos \theta \boldsymbol{d} \overline{\Psi_{M}} \wedge \boldsymbol{d} \Phi_{N} \\
& =i \pi\left(\delta_{n, m-1}+\delta_{n, m+1}\right)\left(n \frac{x_{p(m)}^{\prime}}{a} \Xi_{M, N}^{1}\left(J^{\prime}, J, x^{\prime}, x\right)\right. \\
& \left.+m \frac{x_{q(n)}}{a} \Xi_{M, N}^{1}\left(J, J^{\prime}, x^{\prime}, x\right)\right),
\end{aligned}
$$

and

$$
\begin{aligned}
G_{M, N}^{\bar{\Phi}, \Psi}:= & \int_{\mathcal{D}} r \cos \theta \boldsymbol{d} \overline{\Phi_{M}} \wedge \boldsymbol{d} \Psi_{N} \\
= & i \pi\left(\delta_{n, m-1}+\delta_{n, m+1}\right)\left(n \frac{x_{p(m)}}{a} \Xi_{M, N}^{1}\left(J^{\prime}, J, x, x^{\prime}\right)\right. \\
& \left.+m \frac{x_{q(n)}^{\prime}}{a} \Xi_{M, N}^{1}\left(J, J^{\prime}, x, x^{\prime}\right)\right)
\end{aligned}
$$

\section{Appendix B}

In this appendix the derivation of the expressions for $\Upsilon_{N}(t, z)$ in (106), (107), (108), (109), and $\mathcal{A}_{\sigma}(t, z), \mathcal{B}_{\sigma}(t, z)$ in (119), (120), (121), (122) is outlined. Since

$$
\begin{gathered}
\int_{\Sigma} d z f(z) \delta^{\prime}\left(z-z_{0}\right)=-f^{\prime}\left(z_{0}\right) \\
\int_{\Sigma} d z f(z) \delta\left(z-z_{0}\right)=f\left(z_{0}\right)
\end{gathered}
$$

for a smooth function $f$ and $z_{0}$ contained within $\Sigma \subset \mathbb{R}$, double integrals involving smooth functions and $\delta$ or $\delta^{\prime}$ can be reduced to a single integral. For $\Upsilon_{N}, \mathcal{A}_{\sigma}$ and $\mathcal{B}_{\sigma}$, the triangular integration range defined in the $\left(t^{\prime}, \zeta\right)$ plane by $0 \leq t^{\prime} \leq t$ and $z-c\left(t-t^{\prime}\right) \leq \zeta \leq z+c\left(t-t^{\prime}\right)$ is reduced to a line segment specified by the support, $t^{\prime}=\zeta / v$, of the distribution $\delta(\zeta-v t)$. The slope of this line can only vary between $\frac{1}{c}$ and infinity. The value of this slope determines which side of the bounding triangle the line intersects: see Fig 6 (a) and Fig 6(b). For $v>0$ if $z>v t$ the line segment 
intersects the line $t^{\prime}=\left(t-\frac{z}{c}\right)+\frac{\zeta}{c}$ at $\left(\zeta=\frac{z-c t}{1-\frac{c}{v}}, t^{\prime}=\frac{z-c t}{v-c}\right)$. If $z<v t$ the line segment intersects the line $t^{\prime}=\left(t+\frac{z}{c}\right)-\frac{\zeta}{c}$ at $\left(\zeta=\frac{z+c t}{1+\frac{c}{v}}, t^{\prime}=\frac{z+c t}{v+c}\right)$. When either

$$
z \leq-c t, \quad \text { or } \quad z \geq c t,
$$

there is no intersection of the line $t^{\prime}=\zeta / v$ with the triangle and so $\Upsilon_{N}=0$ yielding (106) and (109). When

$$
-c t<z<c t
$$

there is an intersection of the line segment and the triangle, as shown in Fig [6(a), since $1 / v>1 / c$.

If

$$
\mathcal{K}(t, z):=\int_{0}^{t} d t^{\prime} \int_{z-c\left(t-t^{\prime}\right)}^{z+c\left(t-t^{\prime}\right)} d \zeta \delta^{\prime}(\zeta-v t) f\left(t, z \mid t^{\prime}, \zeta\right)
$$

and

$$
\mathcal{J}(t, z):=\int_{0}^{t} d t^{\prime} \int_{z-c\left(t-t^{\prime}\right)}^{z+c\left(t-t^{\prime}\right)} d \zeta \delta(\zeta-v t) f\left(t, z \mid t^{\prime}, \zeta\right)
$$

it follows that if $-c t<z \leq v t$

$$
\mathcal{J}=\int_{0}^{t_{+}^{\prime}(t, z)} d t^{\prime} f\left(t, z \mid t^{\prime}, v t^{\prime}\right)
$$

and

$$
\mathcal{K}=-\left.\int_{0}^{t_{+}^{\prime}(t, z)} d t^{\prime} \frac{\partial f}{\partial \zeta}\right|_{\zeta=v t^{\prime}}\left(t, z \mid t^{\prime}, \zeta\right)
$$

where

$$
t_{+}^{\prime}(t, z):=\frac{t+z / c}{1+v / c} .
$$

Similarly if $v t \leq z<c t$

$$
\mathcal{J}=\int_{0}^{t_{-}^{\prime}(t, z)} d t^{\prime} f\left(t, z \mid t^{\prime}, v t^{\prime}\right)
$$

and

$$
\mathcal{K}=-\left.\int_{0}^{t_{-}^{\prime}(t, z)} d t^{\prime} \frac{\partial f}{\partial \zeta}\right|_{\zeta=v t^{\prime}} f\left(t, z \mid t^{\prime}, \zeta\right)
$$

where

$$
t_{-}^{\prime}(t, z):=\frac{t-z / c}{1-v / c} .
$$

Using the Bessel relation $J_{0}^{\prime}(z)=-J_{1}(z)$ the integrals (107) and (108) follow immediately from (161) and (162). Similarly the integral representations of $\mathcal{A}_{\sigma}(t, z)$ and $\mathcal{B}_{\sigma}(t, z)$ follow from those for $\mathcal{K}$ and $\mathcal{J}$.

\section{Acknowledgments}

The authors are grateful to C Bane, G Bassi, J Ellison, D Jaroszynski, R Li, M Poole, G Stupakov and R Warnock for valuable discussions and to the Cockcroft Institute and EPSRC for financial support for this research which is part of the Alpha-X collaboration. 


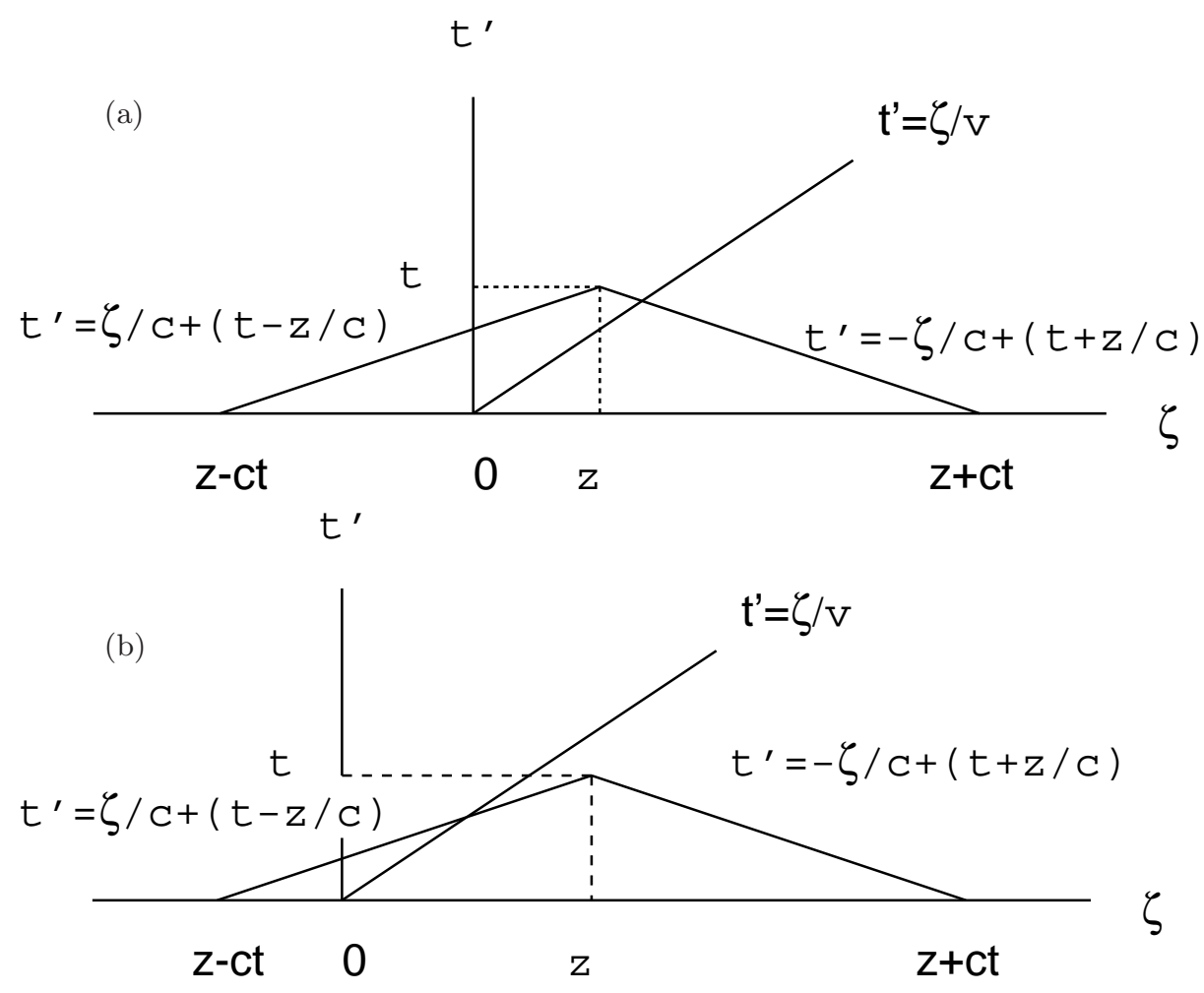

FIG. 6: Integration ranges for $\Upsilon_{N}(t, z)$. (a): $-c t<z \leq v t$, (b): $v t \leq z<c t$.

[1] J Gratus, D Burton and R W Tucker, Multiple Currents in Charged Beams, J. Phys. A: Math. Theor. 40, 811-829, (2007).

[2] J Gratus, D Burton and R W Tucker, The Electrodynamics of self-permeable Charged Continua, ( Proceedings of Conference on Global Integrability and Field Theory, Cockcroft Institute, 2006).

[3] D Burton, J Gratus and R W Tucker, Asymptotic Analysis of Ultra-relativistic Charge, Annals of Physics 322 599-630, (2006).

[4] E L Saldin, E A Schneidmiller and M V Yurkov, On the coherent radiation of an electron bunch moving in an arc of a circle, Nuclear Instruments and Methods in Physics Research A 398, 373 (1997).

[5] G Deschamps, Exterior Differential Forms, (Springer, 1970).

[6] H Flanders, Differential Forms with Application in the Physical Sciences, (N.Y. Academic Press, 1967).

[7] I M Benn and R W Tucker, An Introduction to Spinors and Geometry with Applications in Physics (Adam Hilger, IOP Press, 1987).

[8] T Frankel, The Geometry of Physics, (C.U.P. 2004).

[9] D Burton, A Noble, R W Tucker and D Wiltshire, Twisted Electromagnetic modes and Sagnac Ring Lasers, gr-qc 0411037, Class. Quantum Grav. 22, 909-931, (2005).

[10] M Abramowitz and I A Stegun, Handbook of mathematical functions, ( Dover, 1965).

[11] R W Tucker, On the effects of geometry on guided electromagnetic waves, Thoret. Appl. Mech., 34, 1-50, (2007).

[12] M A Pinsky, Partial Differential Equations and Boundary-Value Problems with Applications, (McGraw-Hill, Inc., 1991).

[13] G Stupakov, C Bane, I Zagorodnov, Optical Approximation in the Theory of Geometric Impedance, SLAC-PUB-12369, 2007

[14] All tensors in this article have dimensions constructed from the SI dimensions $[M],[L],[T]$, $[Q]$ where $[Q]$ has the unit of the Coulomb in the MKS system. We adopt $[g]=\left[L^{2}\right],[G]=[j]=[Q],[F]=[Q] / \epsilon_{0}$ where the permittivity of free space $\epsilon_{0}$ has the dimensions $\left[Q^{2} T^{2} M^{-1} L^{-3}\right]$ and $c_{0}$ denotes the speed of light in vacuo.

[15] If $U \neq V$ but at an event $p$ in spacetime their integral curves share the same tangent vector then it is sometimes said that $V$ is instantaneously at rest at $p$ with respect to the timelike frame $U$.

[16] i.e. $e^{0} \wedge e^{1} \wedge e^{2} \wedge e^{3} \neq 0$.

[17] When $\hat{\beta}$ is independent of $\xi^{3}$ (i.e. $\mathcal{L}_{\frac{\partial}{\partial \xi^{3}}} \hat{\beta}$ it is unnecessary to distinguish notationly between $\boldsymbol{d}$ and $\hat{\boldsymbol{d}}$.

[18] Note that $L_{M}^{H(1)}$ and $S_{M}^{H(1)}$ come from $\overline{\rho_{N}^{(0) \prime}}$ and $\overline{\rho_{N}^{(0)}}$, respectively.

[19] The terms proportional to $L_{M}^{E(1)}, P_{M}^{E(1)}, R_{M}^{E(1)}$ and $S_{M}^{E(1)}$ arise from $\overline{\rho_{N}^{(0) \prime}}, \overline{\dot{\rho}_{M}^{(1)}},\left\{\rho^{(1) \prime}-\left(\rho^{(0)} \kappa_{0}^{\prime}+\rho^{(0) \prime} \kappa_{0}\right) r \cos \theta\right\}$ and $\overline{\rho_{N}^{(0)}}$, 
respectively.

[20] On the test spectator particle worldline, $z=c t-\widetilde{s}$, so one may use this relation to express the longitudinal wake potential as an integral over the worldline parameter $t$ rather than $z$. 